\title{
OPTION PRICING WITH LINEAR MARKET IMPACT AND NONLINEAR BLACK-SCHOLES EQUATIONS
}

\author{
BY GREGOIRE LOEPER \\ Monash University
}

We consider a model of linear market impact, and address the problem of replicating a contingent claim in this framework. We derive a nonlinear Black-Scholes equation that provides an exact replication strategy.

This equation is fully nonlinear and singular, but we show that it is well posed, and we prove existence of smooth solutions for a large class of final payoffs, both for constant and local volatility. To obtain regularity of the solutions, we develop an original method based on Legendre transforms.

The close connections with the problem of hedging with gamma constraints [SIAM J. Control Optim. 39 (2000) 73-96, Math. Finance 17 (2007) 59-80, Ann. Inst. H. Poincaré Anal. Non Linéaire 22 (2005) 633-666], with the problem of hedging under liquidity costs [Finance Stoch. 14 (2010) 317$341]$ are discussed. The optimal strategy and associated diffusion are related with the second-order target problems of [Ann. Appl. Probab. 23 (2013) 308347], and with the solutions of optimal transport problems by diffusions of [Ann. Probab. 41 (2013) 3201-3240].

We also derive a modified Black-Scholes formula valid for asymptotically small impact parameter, and finally provide numerical simulations as an illustration.

\section{CONTENTS}

1. Introduction . . . . . . . . . . . . . . . . . . . . . . . . . . . . 2665

1.1. Order book modelling . . . . . . . . . . . . . . . . . . . . . 2666

1.2. Motivations and links with previous works _ . . . . . . . . . . . . . . 2667

1.3. The pricing equation . . . . . . . . . . . . . . . . . . . . . . . . . . 2669

1.4. Second-order target problems and optimal transport by diffusions . . . . . . . . . . 2670

1.5. Organization of the paper . . . . . . . . . . . . . . . . . . . . 2671

2. Heuristics . . . . . . . . . . . . . . . . . . . . . . . . . . . . . . 2672

2.1. Modified spot dynamics . . . . . . . . . . . . . . . . . . . . . . . 2672

2.2. The pricing equation . . . . . . . . . . . . . . . . . . . . . . 2674

2.3. Remarks . . . . . . . . . . . . . . . . . . . . . . . . . . . . . . . 2674

3. Time continuous formulation of the problem . . . . . . . . . . . . . . . . . 2676

3.1. The pricing equation . . . . . . . . . . . . . . . . . . . . . . 2678

3.2. The intensity dependent impact . . . . . . . . . . . . . . . . . . . . . . . . . 2679

3.3. The verification theorem . . . . . . . . . . . . . . . . . . . . . 2679

3.3.1. The risk-neutral dynamic . . . . . . . . . . . . . . . . . . . 2680

4. Dual formulation of the problem . . . . . . . . . . . . . . . . . . 2681

Received August 2016; revised July 2017.

MSC2010 subject classifications. 91G20, 93E20, 35K55, 49L20.

Key words and phrases. Hedging, price impact, fully nonlinear parabolic equations. 
4.1. Relation to a problem of optimal transport . . . . . . . . . . . . . . . . 2683

4.2. Interpretation in terms of robust hedging . . . . . . . . . . . . . . . . . 2683

5. Smooth solutions via Legendre-Fenchel transform . . . . . . . . . . . . . . . . . . . . 2684

5.1. Notation . . . . . . . . . . . . . . . . . . . . . . . . . . 2684

5.2. Some facts about the Legendre-Fenchel transform . . . . . . . . . . . . . . . . . 2684

5.3. Transformation of the pricing equation via Legendre transforms . . . . . . . . . . . . 2685

Construction of the terminal value for the transformed equations . . . . . . . . 2686

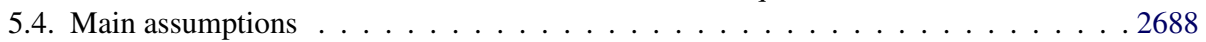

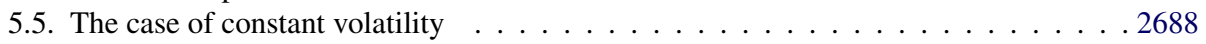

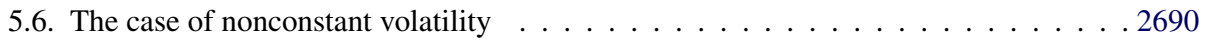

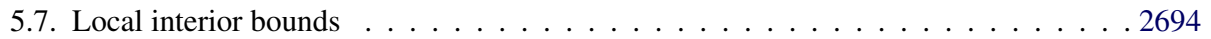

5.8. Initial regularity . . . . . . . . . . . . . . . . . . . . . . . . . . . . . . . . . . . . . . . . . . . . . . . . . . . . . .

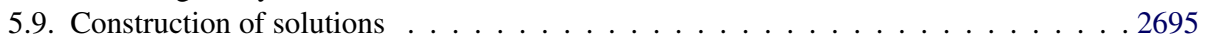

5.10. Existence and uniqueness results . . . . . . . . . . . . . . . . . . . . . . . . . . . . . . . . . . . . . . . . . . . . . . . . . . . . . .

6. Representation of the solution . . . . . . . . . . . . . . . . . . . . . 2697

6.1. A maximum principle for the second derivative . . . . . . . . . . . . . . . . . 2699

6.2. Dual representation formula and martingale property under $\mathbb{P} \ldots$. . . . . . . . . . 2701

6.3. Black-Scholes representation formula, and explicit formulation of the density . . . . 2707

7. A Black-Scholes-Legendre formula . . . . . . . . . . . . . . . . . . . . 2710

7.1. Formal computations . . . . . . . . . . . . . . . . . . 2710

7.2. First-order expansion of the solution . . . . . . . . . . . . . 2712

8. Numerical simulations . . . . . . . . . . . . . . . . . . . . . . . . . . . 2715

Appendix A: Proof of Propositions 5.9 and $5.10 \ldots \ldots$. . . . . . . . . . . . . . 2716

A.1. Preliminary lemmas . . . . . . . . . . . . . . . . . . 2716

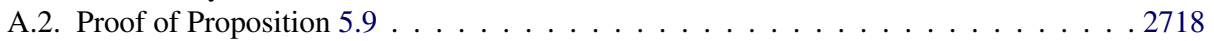

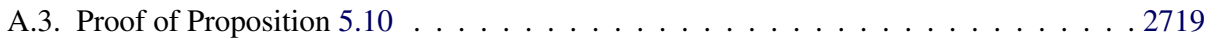

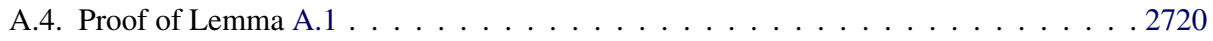

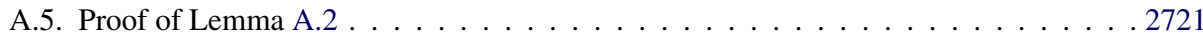

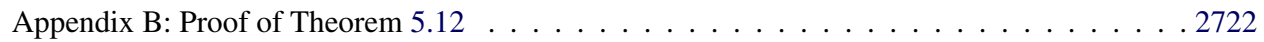

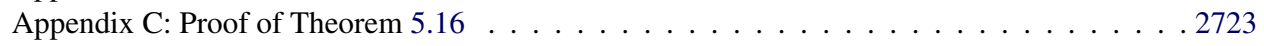

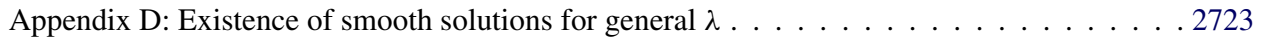

Acknowledgements . . . . . . . . . . . . . . . . . . . . . . . . 2724

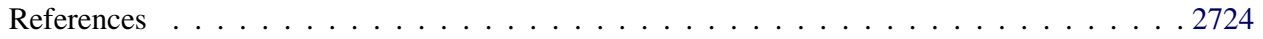

1. Introduction. This paper is about the derivation and mathematical analysis of a pricing model that takes into account the market impact of the option's hedger, that is, the feedback mechanism between the option's delta-hedging and the price dynamics. The starting point of our modelling will be the Black-Scholes model with local volatility, where the stock's price $S_{t}$ follows the well-known stochastic differential equation

$$
\frac{d S_{t}}{S_{t}}=v_{t} d t+\sigma\left(t, S_{t}\right) d W_{t},
$$

$W_{t}$ being a standard Brownian motion, $v_{t}$ an adapted process and the exogenous volatility $\sigma$ depends on $t$ and $S_{t}$. We will throughout the paper assume a linear market impact: each order to buy $N$ stocks impacts the stock's price by $\lambda N S^{2}$ $(\lambda \geq 0)$. This scaling, that will be discussed hereafter, means that the impact in 
terms of relative price move depends on the amount of stock traded expressed in currency, hence $\lambda$ is homogeneous to the inverse of the currency, that is, percent per dollar:

$$
\text { Order to buy } N \text { stocks } \quad \Longrightarrow \quad S \rightarrow S(1+\lambda N S) \text {. }
$$

As we are interested only in continuous trading strategies, we only require this behaviour to hold at leading order in $N$, we do not make any assumptions on the market impact of large orders. We will examine several situations where $\lambda$ is either constant, or can be also a function of the solution itself.

The literature devoted to the study of the market impact itself is quite vast, and it is more frequent to find an impact that varies as a power law of the size of the trade (see, e.g., [2]). Although our linear approach is clearly not the most realistic in terms of market microstructure, it has the advantage of avoiding arbitrage opportunities, as well as not being sensitive to the hedging frequency: in a nonlinear model, splitting an order in half would induce a different market response compared to executing a single trade, a situation that we want to avoid here, as we aim at deriving a time continuous formulation.

In addition, we assume that the market impact is permanent: there is no relaxation following the immediate impact, where the price goes back partially to its pre-trade value. The case with relaxation has been studied in a companion paper [1].

1.1. Order book modelling. From an order book perspective, our market impact model consists in assuming an order book with continuous positive density around the mid-price, and where following a market-order starting at price $S$ (e.g., say a buy order), where liquidity has been consumed up to $S+\delta S$, the new midprice becomes $S+\delta S$, and the liquidity removed between $S$ and $S+\delta S$ is instantaneously refilled with buy orders (or sell orders if the price had moved down after a sell order). Clearly, this model is a great simplification of what is actually observed in real markets (see [37] for instance), but as we will show this simple approach is enough to obtain a nontrivial modification of the usual Black-Scholes equation. More precisely, consider a static order book, parametrized by a mid price $\bar{S}$ and a supply intensity $\mu(t, s)$ in the following way: the number of stocks available for purchase between $S$ and $S+d S$ is equal to $\mu(t, S) d S$. (Of course in the continuous setting, the number of stocks might not be an integer.)

When executing a market order for an amount $A$ (expressed in currency), the order book will be consumed up to $\bar{S}(1+\varepsilon(A))$, where $\varepsilon(A)$ satisfies

$$
A=\int_{\bar{S}}^{\bar{S}(1+\varepsilon(A))} \mu(t, s) s d s,
$$

while the number of stocks purchased will be

$$
N=\int_{\bar{S}}^{\bar{S}(1+\varepsilon(A))} \mu(t, s) d s .
$$


We define the (local) liquidity $L(t, \bar{S})$ at $\bar{S}$ by

$$
L(t, \bar{S})=\lim _{A \rightarrow 0} \frac{A}{\varepsilon(A)}=\mu(t, \bar{S}) \bar{S}^{2} .
$$

The local liquidity $L$ is such that the sum (in currency) of all the sell (resp., buy) limit orders values between $\bar{S}$ and $\bar{S}(1+\varepsilon)$ [resp., $\bar{S}(1-\varepsilon)$ ] equals $L(t, \bar{S}) \varepsilon+o(\varepsilon)$.

Simple calculations show that the scaling (1) implies that, at the leading order in $A, \varepsilon(A)=\lambda N \bar{S}=\lambda A$, hence that $L(t, S) \equiv \lambda^{-1}$. We believe that the quantity $L$ is the good measure of a stock's liquidity, as it should be invariant under operations such as stock splitting (or reverse splitting).

An important point to notice too is that, still assuming a market order for an amount $A$, at the leading order when $A \rightarrow 0$, the average price of execution is $\bar{S}\left(1+\frac{1}{2} \varepsilon(A)\right)$.

In a subsequent study [1], the authors consider an immediate relaxation of the price after the liquidity is consumed, hence the liquidity of the order book is rebuilt around $\bar{S}(1+\gamma \varepsilon)$ for a certain relaxation factor $\gamma \in[0,1]$. We will only address here the case $\gamma=1$. The choice $\gamma=0$ is the one that has been studied by Cetin, Soner and Touzi in [8]: no permanent market impact, but liquidity costs.

1.2. Motivations and links with previous works. In terms of concrete applications, the problem of derivatives pricing with market impact arises when the delta hedging of the option implies a volume of transactions on the underlying asset that is nonnegligible compared to the average daily volume traded. For example, a well observed effect known as stock pinning arises when the hedger is long of the (convex) option for a large notional, and one observes then a decrease of realized volatility if the stock's price ends near the strike at maturity. In financial terms, the hedger of the option makes a loss if the volatility realizes below its implied value. This stylized fact can be recovered by our pricing equation. Conversely, when selling a convex option for a large notional, a common market practice on derivatives desks is to super-replicate the option by the cheapest payoff satisfying a constraint of gamma (the second derivative of the option), the gamma max being adjusted to the liquidity available on the option's underlying. Hence there are two issues arising here:

- Be able to control a priori the trading volume due to delta-hedging: this approach has been studied in depth in the works by Soner and Touzi [34] and Cheridito, Soner and Touzi [10] that deal with the problem of hedging with gammaconstraints.

- Quantify the liquidity costs induced by the delta-hedging, and incorporate them in the option's price; this has been studied by Cetin, Jarrow and Protter in [7] and by Cetin, Soner and Touzi in [8]. However, these works consider only liquidity costs, and not the effect of permanent market impact. 
Our approach addresses those two issues: first, via the market impact mechanism, it induces a constraint on the gamma when selling a convex payoff, and it constraints the theta (the time derivative of the option) when selling a concave payoff. Thus it recovers two important stylized facts of the gamma constraint approach; moreover, it incorporates liquidity costs in the price. It can be noted that the parabolic operator that we obtain through market impact lies somehow between the gammaconstraint operator of [32] and the liquidity costs operator of [8].

A formal argument shows that, playing on the dependency of the market impact parameter with the solution, one can recover exactly the gamma constraint pricing equation of Cheridito, Soner and Touzi [10], or the liquidity cost equation of Cetin, Soner and Touzi in [8]. This can be seen as closely related to the work of Serfaty and Kohn [23] that recover nonlinear heat equations by stationary games approach. From a financial modelling perspective, this would amount to assume a supply curve for the price of gamma-hedging.

Concerning the mathematical techniques, as opposed to [4, 5, 8, 10, 34], we address the problem of finding exact replication strategies, while the aforementioned works deal with stochastic target problems: find the cheapest trading strategy that super-replicates the final payoff. The solutions of these two problems coincide in general, but may differ in some degenerate cases (see [1, 33]). Apart from introducing the linear market impact model (which has then led to the subsequent studies $[1,4,5])$, the main contribution of this paper is a complete study of a fully nonlinear parabolic equation associated to a new class of stochastic control problems. We will also prove representation formulas that gives some qualitative information about the modified dynamics. Those two results are then used to derive rigorously an asymptotic expansion of the solution for small market impact, leading to a modified Black-Scholes-Legendre formula, as well as a simple and efficient way of computing the market impact effect.

We believe that the techniques used towards obtaining these results are original and of independent interest. As will be discussed hereafter, these results can be seen as related to regularity results concerning porous media equations (see the book by Vazquez [39] for a complete reference), in particular in the case of Fast Diffusion Equations, as well as the papers by Crandall and Pierre [11, 13] about interior regularity for nonlinear diffusions.

The stochastic target problem associated to our model has been studied in two companion papers $[4,5]$, where it is shown that the exact replication strategy is actually the optimal solution for the stochastic target problem. To make a connection between the two results, it is important to notice that through stochastic control techniques, one is able to derive a viscosity formulation of the value function. It is only when this function has enough regularity that one can deduce the optimal strategy from the value function, hence the importance of the question of regularity.

It is important to notice at this stage that two different approaches can be taken when considering the problem of pricing the market impact. One approach takes 
into account the cost of building and liquidating the initial and final hedging portfolios, which is the approach of [4]. Another way of trading, known as covered options, is considered in [5]: the option's buyer provides the seller with the initial hedge (at its marked-to-market value), and receives at maturity the final hedging portfolio. This type of settlement at maturity is called physical settlement, for example, in the case of call option finishing in the money, the buyer would receive at maturity one stock and pay the strike price to the seller. This is a common market practice, either for very large deals and also for cross currency options that are most of the time physically settled. We focus only on the second approach in this paper which leads to a radically different pricing equation compared to the one obtained in [4]. When physical delivery is not possible, another common market practice is to use "asian-in" and "asian-out" variations of the payoff, where the initial strike price and the final fixing depend on the average value of the stock over a certain period. This allows to spread over time the construction and liquidation of the hedging portfolio, and mitigates the market impact of the initial and final transactions, since the option naturally starts and ends with no delta.

Finally, we mention the references $([14,15,24,29,31])$ that also address the problem of option hedging in nonperfect markets with different approaches, leading to different mathematical techniques.

1.3. The pricing equation. As we will see, assuming no interest rates and dividends, the pricing equation that we obtain can be put under the form

$$
\partial_{t} u+\frac{1}{2} \sigma^{2} \frac{s^{2} \partial_{s s} u}{1-\lambda s^{2} \partial_{s s} u}=0
$$

which reads also

$$
\frac{\sigma^{2}}{2 \partial_{t} u}+\frac{1}{s^{2} \partial_{s s} u}=\lambda,
$$

where $\lambda$ can be either constant or dependent of the solution as $\lambda\left(s^{2} \partial_{s s} u\right)$. In this case, we obtain a wide class of fully nonlinear Black-Scholes equations of the form

$$
\partial_{t} u+\frac{1}{2} \sigma^{2} F\left(s^{2} \partial_{s s} u\right)=0
$$

As will be shown, one can derive any parabolic equation of this form through an ad hoc choice of $\lambda$, as long as $F(\gamma) \geq \gamma$ and $F(0)=0$.

The case of equation (2) is quite challenging from the mathematical perspective: the operator $\partial_{s s} u \rightarrow F\left(s^{2} \partial_{s s} u\right)$ is not uniformly elliptic (when $\partial_{s s} u$ goes to $-\infty$ ), and is singular (when $s^{2} \partial_{s s} u$ goes to $\lambda^{-1}$ ), hence the standard regularity theory does not apply. Still, by developing ad hoc techniques, we will be able to show interior (i.e., not relying on a smooth terminal payoff) regularity of solutions for 
constant $\lambda$ and global (i.e., assuming a smooth terminal condition) regularity for nonconstant $\lambda$.

Let us rewrite equation (2) as follows:

$$
\begin{gathered}
\partial_{t} u+\frac{1}{2} \sigma^{2} F\left(s^{2} \partial_{s s} u\right)=0, \\
F(\gamma)=\frac{\gamma}{1-\lambda \gamma}=\frac{1}{\lambda}\left(-1+\frac{1}{1-\lambda \gamma}\right) .
\end{gathered}
$$

The link with nonlinear diffusions appears when one differentiates twice the equation: assuming that $\sigma$ is constant one then obtains for $\beta=1-\lambda s^{2} \partial_{s s} u$,

$$
\partial_{t} \beta-\frac{\sigma^{2} s^{2}}{2} \partial_{s s}\left(\frac{1}{\beta}\right)=0,
$$

which can be seen as a log-normal version of the fast diffusion equation (see [39])

$$
\partial_{t} u-\Delta\left(\frac{1}{u}\right)=0
$$

1.4. Second-order target problems and optimal transport by diffusions. There is also a clear connection between this work and the work by Soner, Touzi and Zhang [35] about dual formulation of second-order target problems, and also with the problem of optimal transport by controlled diffusions studied in [36]. Indeed observe that the elliptic operator $F(\gamma)$ in (2) is convex, and then applying the reasoning of [35] based on the Legendre transform representation of $F$, one can show formally that a solution to (2) will also be solution of the following variational problem:

$$
u(t, s)=\sup _{a \in \mathcal{A}_{T}} \mathbb{E}\left\{\Phi\left(S_{T}^{a}\right)-\frac{1}{2 \lambda} \int_{t}^{T}\left(a_{t^{\prime}}^{1 / 2}-\sigma\left(t^{\prime}, S_{t^{\prime}}^{a}\right)\right)^{2} d t^{\prime}\right\},
$$

where $S^{a}$ starts from $s$ at time $t$ and follows $d S_{r}^{a}=a_{r}^{1 / 2} S_{r}^{a} d W_{r}, W_{r}$ is a Brownian motion, $\mathcal{A}_{T}$ is the set of all bounded positive adapted diffusion processes on $[t, T]$, and $\sigma(t, s)$ is given and positive. This can be seen as a stochastic version of the Hopf-Lax formula,

$$
u(t, s)=\sup _{\gamma \in C^{1}([0, T]), \gamma(t)=s}\left\{\Phi(\gamma(T))-\int_{t}^{T} H\left(\partial_{t} \gamma\left(t^{\prime}\right)\right) d t^{\prime}\right\},
$$

which is a representation formula for viscosity solutions to the Hamilton-Jacobi equation

$$
\partial_{t} u+H^{*}\left(\partial_{x} u\right)=0,
$$

where $H^{*}$ is the Legendre transform of $H$, see [3]. The fact that, in general, the solution of a variational problem like (3) is indeed the viscosity solution of an 
associated parabolic equation [which here would be (2)] has been studied in [35]. In this particular case, we will be able to actually characterize the optimal $\hat{a}$ in (3), and show that the optimal diffusion $S^{\hat{a}}$ is a martingale up to time $T$ under mild conditions, which allow $\hat{a}$ to be unbounded.

Then, still reasoning formally, $S^{\hat{a}}$ transports its initial distribution on its final distribution minimizing the transport cost

$$
\mathbb{E}\left(\frac{1}{2} \int_{0}^{T}\left(a_{t}^{1 / 2}-\sigma\left(t, S_{t}\right)\right)^{2} d t\right),
$$

which is a particular case of the problem studied in [36]. More generally, the link between the variational problem (3) and optimal transport arises through the use of Kantorovitch duality; see [6, 20,21] and also [40] for an overview on optimal transport, and also [27] for further extensions to nonlinear variational problems.

The regularity results that we obtain here will provide the regularity of the optimizers in the aforementioned problems.

1.5. Organization of the paper. The rest of the paper is organized as follows: in the next section (Section 2), we provide a heuristic derivation of the pricing equation, and discuss its practical relevance for financial modelling.

Section 3 gives the time continuous formulation of the problem as a system of stochastic differential equations, and shows that the pricing equation (2) actually leads to an exact replication strategy when it admits a smooth solution; see Theorem 3.2.

Section 4 establishes the connection between the solution of (2) and the problem (3), and discusses also connections with optimal transport and robust hedging.

Section 5 contains the regularity results for equation (2). Theorems 5.6, 5.8 and 5.12, contain the main a priori estimates for solutions to (2), that hold under mild conditions on the final payoff. These results lead then to the existence, regularity and uniqueness of the solutions to (2): Theorem 5.15.

In Section 6, we study the dynamic of the stock's price induced by the replication strategy. One of the main results of this section is to show that, under mild conditions on the exogenous volatility $\sigma$ and the terminal payoff $\Phi, S_{t}$ remains a martingale up to maturity, although its log-normal diffusion might not be bounded. We also establish representation formulas for the solution: Theorems 6.10, 6.6 and 6.11. These representation formulas are a probabilistic counterpart to equation (2), and give qualitative insight about the solution. In particular, in the case of constant initial volatility, we establish an explicit formulation of the final distribution of the price at maturity. The results of Section 4 on problem (3) are also extended to the case where the perturbed diffusion might unbounded.

In Section 7, we derive a first-order expansion of the solution for small market impact $(\lambda)$. This leads also to modified Black-Scholes-Legendre formula [see formulas (103), (105), (106)], which can be easily computed either analytically or by 
standard Monte-Carlo simulations. It should formally be also valid in the case of liquidity costs studied in [8].

Section 8 provides, for illustration purposes, a numerical solution of equation (2).

It should be noted that the regularity results of Section 6 play a crucial role in establishing the representation formulas of Section 7 (see in particular the important Proposition 6.3), which are then necessary for rigorously establishing the asymptotic expansion of Section 7.

2. Heuristics. We assume that we have sold an option whose value is $u(t, s)$, and greeks are as usual

$$
\begin{aligned}
& \Delta=\partial_{s} u, \\
& \Gamma=\partial_{s} \Delta, \\
& \Theta=\partial_{t} u .
\end{aligned}
$$

We also introduce the gamma in currency, that is,

$$
\gamma=\Gamma s^{2}=\partial_{s s} u s^{2} \text {. }
$$

The strategy that we use is the following: we assume a priori that there exists an exact replication strategy, that consists in holding $\Delta=\partial_{s} u$ stocks. We compute the equation that must be followed by $u$, and the modified dynamics that this strategy implies. We then check that this strategy allows indeed to perfectly replicate the final claim.

2.1. Modified spot dynamics. As discussed in the Introduction, we are in the framework of covered options, hence we start from a delta-hedged portfolio. We assume that the stock's price $S$ moves initially by $d S$, given by

$$
d S=S\left(\sigma d W_{t}+v d t\right)
$$

where $W_{t}$ is a standard Brownian motion, and $d W_{t}$ its increment between $t$ and $t+d t$ (those objects will be introduced more formally later on). A "naive" hedge would be to buy $\Gamma d S$ stocks, but as this order will impact the market, the portfolio will not end up delta-hedged. Assume instead a hedge adjustment of $\Gamma \tilde{d} S$ stocks, and let us find $\tilde{d S}$ such that the spot ends at the final value $S+\tilde{d S}$. Using (1), we write that

$$
\begin{aligned}
& S_{\text {after re-hedging }}-S_{\text {before re-hedging }} \\
& \quad=\lambda S_{\text {before re-hedging }}^{2} \text { Number of stocks bought to re-hedge. }
\end{aligned}
$$

Note that $\left(\lambda S^{2}\right)$ before re-hedging means that $\lambda S^{2}$ is evaluated at $S+d S$ (after the initial move of $S$ ):

$$
\tilde{d S}-d S=[\Delta(t+d t, S+\tilde{d S})-\Delta(t, S)] \lambda(S+d S)^{2} .
$$


This identity expresses the fact that the number of titles bought is $\Delta(S+\mu d S)-$ $\Delta(S)$, hence that the portfolio is delta-hedged at the end of the trade. Performing a Taylor expansion leads to

$$
\begin{aligned}
\tilde{d S}= & d S+\Gamma \lambda S^{2} \tilde{d S} \\
& +\partial_{t} \Delta \lambda S^{2} d t+\frac{1}{2} \partial_{s S} \Delta \lambda S^{2}(\tilde{d} S)^{2}+\Gamma \partial_{s}\left(\lambda S^{2}\right)(\tilde{d S} d S)+o(d t) .
\end{aligned}
$$

Plugging the ansatz

$$
\frac{\tilde{d} S}{S}=\mu \frac{d S}{S}+\tilde{v} d t
$$

into the above equation, together with (6), we find that

$$
\mu=\frac{1}{1-\lambda \gamma}
$$

and the expression of $\tilde{v}$ follows. Note also that

$$
\tilde{d S}=d S+\lambda S^{2} d \Delta+\partial_{S}\left(\lambda S^{2}\right)(d \Delta d S)+o(d t),
$$

which is consistent with the derivation established in [4], Proposition 1 and expressed later in (19), (22).

Then the value $V$ of the hedging portfolio containing $\Delta(t, S)$ stocks at the beginning of time $t$, and $\Delta(t+d t, S+\tilde{d S})$ stocks at time $t+d t$ evolves as

$$
d V=\Delta \tilde{d S}+\mathcal{R}
$$

and $\mathcal{R}$ is the profit realized during the re-hedging. In a perfect frictionless market, this term is zero, as one buys $d \Delta$ stocks at a price $S+d S$, and the "post-re-hedge" value of the stocks is $S+d S$. To compute $\mathcal{R}$, we recall that during the execution:

(9) Immediate impact of an order to buy $N$ stocks $\Longrightarrow S \rightarrow S(1+\lambda N S)$,

(10) Average execution price $=S\left(1+\frac{1}{2} \lambda N S\right)$.

The computation of $\mathcal{R}$ then yields at the leading order

$$
\begin{aligned}
\mathcal{R} & =N(\text { Final price of the stocks bought }- \text { Average execution price }) \\
& =N\left(S(1+\lambda N S)-S\left(1+\frac{1}{2} \lambda N S\right)\right) \\
& =\frac{1}{2} \lambda N^{2} S^{2}
\end{aligned}
$$

with $N=\Gamma \tilde{d S}$. Therefore, one obtains at the leading order

$$
d V=\Delta \tilde{d S}+\frac{1}{2} \lambda S^{2}(\Gamma \tilde{d S})^{2}+o(d t)
$$


2.2. The pricing equation. We now assume that the option is sold at its fair price, hence $d V=d u$. Then we have as $S$ moves to $S+\tilde{d S}$,

$$
d u=\partial_{t} u d t+\partial_{s} u \tilde{d} S+\frac{1}{2} \partial_{s s} u(\tilde{d} S)^{2}+o(d t),
$$

and we thus get

$$
\partial_{t} u d t+\frac{1}{2}(\tilde{d S})^{2}\left[\partial_{s s} u-\lambda\left(\partial_{s S} u\right)^{2} S^{2}\right]=o(d t) .
$$

At the leading order, we have $\tilde{d S}=\mu S \sigma d W_{t}$, where $\mu$ is given by (8), and following Itô's formula one can replace $(\tilde{d S})^{2}$ by $\mu^{2} \sigma^{2} S^{2} d t$ in the previous Taylor expansion, and obtain

$$
\begin{aligned}
\partial_{t} u+\frac{1}{2} \sigma^{2} F(\gamma) & =0, \\
F(\gamma) & =\frac{\gamma}{1-\lambda \gamma}, \\
\gamma & =s^{2} \partial_{s s} u .
\end{aligned}
$$

2.3. Remarks. Note that if we change assumption (10) in

$$
\text { Average execution price }=S(1+\lambda N S) \text {, }
$$

then we obtain the equation

$$
\partial_{t} u+\frac{1}{2} \sigma^{2} \frac{\gamma}{(1-\lambda \gamma)^{2}}=0
$$

as found in [26]. This equation is not parabolic however.

Again, we are in the framework of covered options, hence we start from a deltahedged portfolio, and deliver at maturity a mix of cash and stocks of market value $u\left(T, S_{T}\right)$ to the option's buyer. We do not liquidate the hedge at maturity (which would cause the spot to jump).

Remember that $\gamma$ is computed with respect to the option the portfolio is short of, hence $\gamma>0$ when one sells a call, for example. Assuming that $\lambda \gamma<1$ (to be discussed later), one sees that $\mu>1$ : as expected the hedger increases the volatility by buying when the spot rises, and selling when it goes down.

One can also reach the conclusion (8) by following an iterative hedging strategy: after the initial move $S \rightarrow S+d S$, the hedge is adjusted "naively" by $\Gamma d S$ stocks, which then impacts the price by $d S_{2}=\lambda S^{2} \Gamma d S$. A second hedge adjustment of $\Gamma d S_{2}$ is done, which in turn impacts the price, and so forth. One can check that the final spot move is thus the sum of the geometric sequence

$$
d S\left(1+\lambda S^{2} \Gamma+\left(\lambda S^{2} \Gamma\right)^{2}+\cdots\right)=\frac{d S}{1-\lambda \gamma} .
$$


When $\lambda S^{2} \Gamma \geq 1$, the sequence is not converging: indeed, the market impact due to a hedge adjustment is larger than the price move that initiated the adjustment. The hedger runs after the spot, without being able to eventually reach a price where he is hedged, and the spot runs away to infinity or to 0 . In that case, believing in the model, when $1-\lambda \gamma<0$, when the spot moves up, one should sell stocks instead of buying, because the market impact will make the spot go down. One relies on the market impact to take back the spot at a level where the portfolio is hedged. This situation is clearly nor realistic, nor acceptable from a trading perspective. It seems therefore reasonable to add to complement the pricing equation with the constraint that at each time $\lambda \gamma \leq 1$.

On the other hand, if we consider a smooth function that satisfies for all time $t \in[0, T]$ the constraint

$$
1-\lambda s^{2} \partial_{s s} u(t, s) \leq 1-\varepsilon \quad \text { for some } \varepsilon>0,
$$

solution of (12), (13) with terminal payoff $u(T, s)=\Phi(s)$, the trading strategy that holds $\Delta_{t}=\partial_{s} u\left(t, S_{t}\right)$ stocks at each time $t$ yields an exact replication strategy (see Theorem 3.2).

The heuristic derivation has been presented in the case of a constant volatility, but could be adapted to a local or stochastic volatility model.

The function $F$ is increasing, thus equation (12) is of parabolic type and, therefore, a priori well posed, but it is fully nonlinear, and not uniformly parabolic. The question of the existence, regularity and uniqueness of solutions does not follow from standard parabolic theory and needs an ad hoc treatment, which is one the results of this paper.

Another (informal) way to see the constraint $\lambda \gamma \leq 1$ imposed on $u$ is to consider instead of $F$

$$
\begin{aligned}
F(\gamma) & =\frac{\gamma}{1-\lambda \gamma} & \text { if } \lambda \gamma<1 \\
& =+\infty & \text { otherwise. }
\end{aligned}
$$

The physical interpretation of the singular part is that areas with large positive $\gamma$ (i.e., such as $\lambda \gamma>1$ ) will be quickly smoothed out and will instantaneously disappear, as if the final payoff was smoothed (again this argument will be made rigorous later on). This amounts to replace the solution $u$ by the smallest function greater than $u$ and satisfying the constraint $\left(\lambda s^{2} \partial_{s s} u \leq 1\right.$ ) (a semi-concave envelope, the so-called "face-lifting" in [34]). This is actually a common practice on derivatives trading desks: one replaces then a single call by a strip of calls, in order to cap the $\Gamma$. It can be expressed by turning the system (12), (13) into

$$
\begin{gathered}
\max \left\{\partial_{t} u+\frac{1}{2} \sigma^{2} F(\gamma), \lambda \gamma-1+\varepsilon\right\}=0, \\
F(\gamma)=\frac{\gamma}{1-\lambda \gamma}
\end{gathered}
$$




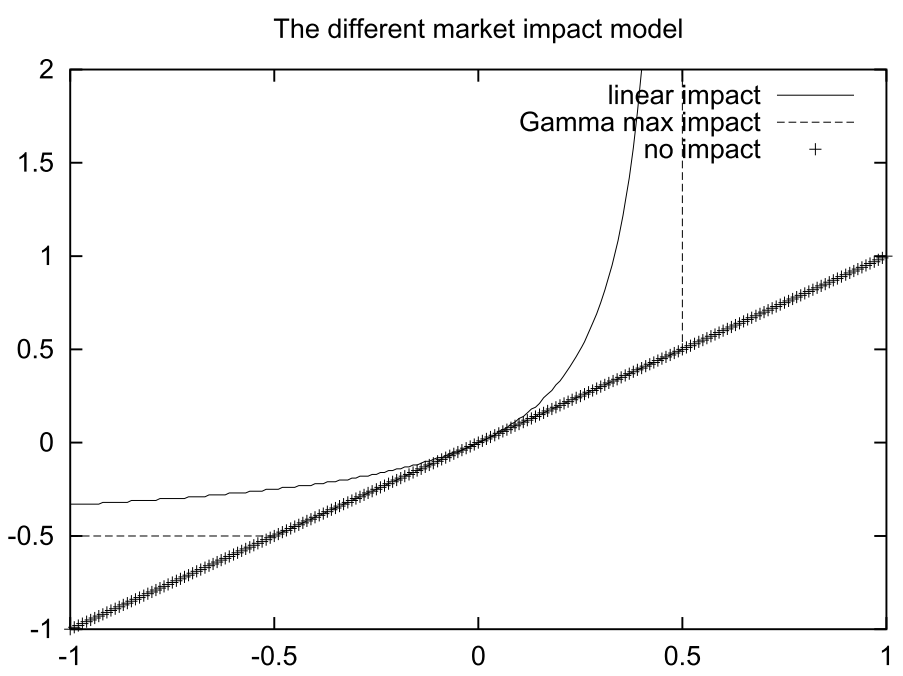

FIG. 1. The function $\Gamma_{c} \rightarrow F\left(\Gamma_{c}\right)$ used for the different models.

for some $\varepsilon>0$, still with $\gamma=s^{2} \partial_{s s} u$. Under this formulation, the problem enters into the framework of viscosity solutions; see [12]. This is the problem that the authors have been addressing in [5].

Note that on the other hand, areas with large negative $\gamma$ (the hedger is buying a convex payoff) would have very little diffusion, which also poses a problem as the equation is not uniformly parabolic any more.

The functions $\Gamma \rightarrow F(\Gamma)$ are represented in Figure 1. The "Gamma max" impact corresponds to pricing equation derived in [34].

3. Time continuous formulation of the problem. We now formulate our problem as a system of stochastic differential equations, as done in $[4,5]$. This formulation is similar to the one of Soner and Touzi [32, 34] of stochastic target problems, or to the formulation of backward stochastic differential equations of [9]. The crucial difference is that here the price (or state) process $S_{t}$ itself has its dynamic modified by the controls.

We consider a probability space $\left(\Omega, \mathbb{F}, \mathbb{P}_{0}\right)$ supporting a standard Brownian motion $W_{t}^{\mathbb{P}_{0}}$ and its associated filtration $\left(\mathcal{F}_{t}\right)_{t \geq 0}$. The drift $v_{t}$ will be a bounded adapted process, and we consider bounded processes (the controls) $a_{t}, \Gamma_{t}$, adapted to the filtration $\mathcal{F}_{t}$. For two semi-martingales $u, v,\langle u, v\rangle_{t}$ denote their covariation (and $\langle u, u\rangle_{t}$ denote the quadratic variation of $u$ ). Both $\sigma$ and $\lambda$ are given; they can be constant or have some dependency, which will be made explicit when needed. We will consider the following system of stochastic differential equations:

$$
\frac{d S_{t}}{S_{t}}=\sigma d W_{t}^{\mathbb{P}_{0}}+\lambda S_{t} d \delta_{t}+v^{\prime} d t,
$$




$$
\begin{aligned}
d \delta_{t} & =a_{t} d t+\Gamma_{t} d S_{t}, \\
d V_{t} & =\delta_{t} d S_{t}+\frac{1}{2} \lambda S_{t}^{2} d\langle\delta, \delta\rangle_{t},
\end{aligned}
$$

where $S$ starts from $S_{0}$ at $t=0$. It has been established rigorously in [4], Proposition 1 , how to obtain this system as the limit of a discrete trading strategy. At time $t$, the hedger holds $\delta_{t}$ units of the risky asset, whose value is $S_{t}$. From equations (18), (19), $S_{t}$ and $\delta_{t}$ are two continuous semi-martingales. Equation (18) states that the spot price is driven by an exogenous source of noise and by the market impact (note the nontrivial modification of the drift process $v$ into $v^{\prime}$ detailed below, which, however, will not affect the pricing equation). Equation (20) reflects our assumptions on the order book (see equation (11), in particular it would change if one assumed a relaxation mechanism after the immediate impact, see [1]). It can also be written as

$$
d V_{t}=\delta_{t} d S_{t}+\frac{1}{2} \lambda \Gamma_{t}^{2} S_{t}^{2} d\langle S, S\rangle_{t}
$$

Combining (19) and (18), one sees right away that

$$
\frac{d S_{t}}{S_{t}}\left(1-\lambda S_{t}^{2} \Gamma_{t}\right)=\sigma d W_{t}^{\mathbb{P}_{0}}+\left(\lambda_{t} S_{t} a_{t}+v_{t}^{\prime}\right) d t,
$$

and, therefore, we obtain, as in the previous heuristics [see equation (8)], that the volatility is multiplied by a factor $\mu$, and is now equal to

$$
\sigma^{\gamma}=\frac{\sigma}{1-\lambda S_{t}^{2} \Gamma_{t}}
$$

As shown in [4] [see also the heuristic derivation (7)], the drift is modified as follows:

$$
v^{\prime}=v+\Gamma S_{t} \sigma^{\gamma} \sigma \partial_{s}\left(\lambda S^{2}\right)
$$

due to the fact that the re-hedge order is placed after the initial exogenous move of $S$, which induces an additional covariance term. This term however does not play any role in the pricing equation. We will assume for now the condition:

$$
\text { for some } \varepsilon>0, \quad 1-\lambda S_{t}^{2} \Gamma_{t} \geq \varepsilon \text {. }
$$

We have the first elementary result.

Proposition 3.1. Let $a_{t}, \Gamma_{t}$ be bounded adapted processes. Assume that $\Gamma_{t}$ satisfies uniformly the condition (23). Then there exists a unique strong solution to (19), (18), (20).

The replication problem is to find a self-financed strategy, hence controls $a_{t}, \Gamma_{t}$, and an initial wealth $V_{0}$ such that

$$
\Phi\left(S_{T}\right)=V_{T}, \quad \text { a.s. }
$$


On the other hand, the super-replication problem of [34] is to find the lowest initial wealth such that there exists an admissible control reaching the target, that is,

$$
\Phi\left(S_{T}\right) \leq V_{T}, \quad \mathbb{P}_{0} \text {-a.s. }
$$

The two problems are not equivalent in all cases, in particular when the exact replication equation is not parabolic (see $[1,33]$ ). In that case, the cheapest way to super-replicate $\Phi$ is not the exact replication (and might not even be unique). We address the super-replication problem with market impact in two companion papers $[4,5]$.

For a smooth function $u(t, s)$ such that $u(T, \cdot)=\Phi$, we consider the strategy given by $\delta(s, t)=\partial_{s} u\left(t, S_{t}\right)$ [and hence $\Gamma_{t}=\partial_{s s} u\left(t, S_{t}\right)$ ]. One obtains for the wealth

$$
V_{T}=V_{0}+\int_{0}^{T} \partial_{s} u d S_{t}+\frac{1}{2} \int_{0}^{T} \lambda\left(\Gamma_{t} S_{t}\right)^{2} d\langle S, S\rangle_{t},
$$

while Itô's formula applied to $u$ reads

$$
u\left(T, S_{T}\right)=u\left(0, S_{0}\right)+\int_{0}^{T} \partial_{s} u d S_{t}+\frac{1}{2} \partial_{s s} u d\langle S, S\rangle_{t}+\partial_{t} u d t
$$

Obviously, a function $u(t, s)$ satisfying

$$
\frac{1}{2} \partial_{s s} u\left(t, S_{t}\right) d\langle S, S\rangle_{t}+\partial_{t} u\left(t, S_{t}\right) d t=\frac{1}{2} \lambda\left(S_{t} \partial_{s s} u\left(t, S_{t}\right)\right)^{2} d\langle S, S\rangle_{t}
$$

looks like the good candidate. We know that

$$
\frac{d S_{t}}{S_{t}}=\frac{\sigma_{t}}{1-\lambda_{t} S_{t}^{2} \partial_{s s} u} d W_{t}^{\mathbb{P}_{0}}+\tilde{v}_{t} d t
$$

for some adapted process $\tilde{v}_{t}$ (that depends on $v$ and on $u$ ). Then the condition on $u$ turns into

$$
\frac{1}{2} \partial_{s s} u \frac{\sigma^{2} s^{2}}{\left(1-\lambda s^{2} \partial_{s s} u\right)^{2}}+\partial_{t} u=\frac{1}{2} \lambda\left(s \partial_{s s} u\right)^{2} \frac{\sigma^{2} s^{2}}{\left(1-\lambda s^{2} \partial_{s s} u\right)^{2}} .
$$

3.1. The pricing equation. We rewrite the above equation as

$$
\partial_{t} u+\frac{1}{2} \sigma^{2} F(\gamma)=0,
$$

with $F$ being given by

$$
F(\gamma)=\frac{\gamma}{1-\lambda \gamma}, \quad \gamma=s^{2} \partial_{s s} u,
$$

and with the terminal condition

$$
u(T, s)=\Phi(S) .
$$


3.2. The intensity dependent impact. Let us now turn to a slightly more general heuristic, by allowing the parameter $\lambda$ to depend on the trading strategy. In the previous approach, we have assumed that the market impact of buying $N$ stocks is, in terms of price, $\lambda N S^{2}$, regardless of the time on which the order is spread. We now assume that $\lambda=\lambda(\gamma)$. The quantity $\gamma=\Gamma s^{2}$ relates to the trading intensity, that is, to the "rate" at which stocks are traded by the option's hedger, hence a gamma dependent market impact can make sense. The system of stochastic differential equations governing the evolution is now given by

$$
\begin{aligned}
\frac{d S_{t}}{S_{t}} & =\sigma d W_{t}^{\mathbb{P}_{0}}+\lambda(\gamma) S_{t} d \delta_{t}+v^{\prime} d t, \\
d \delta_{t} & =a_{t}+\Gamma d S_{t}, \\
d V_{t} & =\delta_{t} d S_{t}+\frac{1}{2} \lambda(\gamma) S_{t}^{2} d\langle\delta, \delta\rangle_{t},
\end{aligned}
$$

still with $\gamma=\Gamma s^{2}$. Then the pricing equation remains (24), but now $\lambda=\lambda(\gamma)$. For the equation to be parabolic, we need to have

$$
F(\gamma)=\frac{\gamma}{1-\lambda(\gamma) \gamma}
$$

nondecreasing. We have

$$
F^{\prime}=\frac{1+\lambda^{\prime} \gamma^{2}}{(1-\lambda \gamma)^{2}}
$$

hence the condition on $\lambda$ is that

$$
1+\lambda^{\prime} \gamma^{2}>0
$$

This case is discussed in more detail in Appendix D.

3.3. The verification theorem. We conclude this section by stating a verification result and a representation formula for solutions to the replication problem. We first introduce three versions of condition (16):

$$
\lambda \gamma \leq 1
$$

the strict version of (31),

$$
\lambda \gamma<1
$$

and the uniform bound

$$
\lambda \gamma \leq 1-\varepsilon \quad \text { for some } \varepsilon>0,
$$

and we recall that $\gamma=s^{2} \partial_{s s} u$. 
3.3.1. The risk-neutral dynamic. We consider on $(\Omega, \mathbb{F})$ a probability measure $\mathbb{P}$ and $W^{\mathbb{P}}$ a $\mathbb{P}$-Brownian motion, and consider the solution for $r \in[t, T]$ of

$$
\begin{aligned}
\frac{d S_{r}^{t, s}}{S_{r}^{t, s}} & =\sigma_{r}^{\gamma} d W_{r}^{\mathbb{P}}, \\
\sigma_{r}^{\gamma} & =\frac{\sigma\left(r, S_{r}^{t, s}\right)}{1-\lambda \gamma\left(t, S_{r}^{t, s}\right)}, \\
S_{t}^{t, s} & =s .
\end{aligned}
$$

When $t=0, s=S_{0}$ we might just write $S_{r}, r \geq 0$ instead of $S_{r}^{0, S_{0}}$.

THEOREM 3.2. Let $u$ be a $C^{1,3}\left([0, T] \times \mathbb{R}^{+}\right)$smooth solution of (24), (25), (26) satisfying (33). Assume also that:

(i) $\sigma, \sigma^{-1}, s \partial_{s} \sigma(t, s), \gamma$ are bounded,

(ii) $\lambda, s \partial_{s}(\lambda(\gamma))$ are bounded.

Then there exists a strong solution $\left(S_{t}, \delta_{t}, V_{t}\right)$ to $(27),(28),(29)$ on $[0, T]$ with $\delta_{t}=\partial_{s} u\left(t, S_{t}\right), V_{t}=u\left(t, S_{t}\right)$. The evolution of $S$ under $\mathbb{P}_{0}$ is given by

$$
\begin{aligned}
\frac{d S_{t}}{S_{t}} & =\sigma^{\gamma} d W_{t}^{\mathbb{P}_{0}}+v^{\gamma} d t \\
v^{\gamma} & =v^{\prime}-\frac{\sigma^{2} \lambda \gamma}{(1-\lambda \gamma)^{2}}-\frac{1}{2} \partial_{s}\left(\sigma^{2}\right) \frac{\lambda s \gamma}{1-\lambda \gamma}-\frac{1}{2} \partial_{s}(\lambda(\gamma)) \frac{\lambda s \sigma^{2} \gamma^{2}}{(1-\lambda \gamma)^{2}},
\end{aligned}
$$

with $v^{\prime}$ defined in (22), $\sigma^{\gamma}$ defined in (35).

There holds $\mathbb{P}_{0}$-almost surely,

$$
V_{T}=u\left(0, S_{0}\right)+\int_{0}^{T} \partial_{s} u d S_{t}+\frac{1}{2} \int_{0}^{T} \lambda \frac{\sigma^{2} \gamma^{2}}{(1-\lambda \gamma)^{2}} d t=\Phi\left(S_{T}\right) .
$$

There exists $\mathbb{P}$ absolutely continuous with respect to $\mathbb{P}_{0}$ and $W^{\mathbb{P}}$ a $\mathbb{P}$-Brownian motion such that $S$ is a strong solution to (34), (35), (36) under $\mathbb{P}$, and there holds

$$
u\left(0, S_{0}\right)=\mathbb{E}^{\mathbb{P}}\left(\Phi\left(S_{T}\right)-\frac{1}{2 \lambda} \int_{0}^{T}\left(\sigma^{\gamma}\left(t, S_{t}\right)-\sigma\left(t, S_{t}\right)\right)^{2} d t\right) .
$$

In particular, the results of the theorem hold if $\lambda$ is constant and $\Phi, \sigma$ satisfy the assumptions (i) to (v) of Theorem 5.8 .

Proof. Plugging $\delta_{t}=\partial_{s} u\left(t, S_{t}\right)$ into (19), (18), (20) yields (37). The smoothness of $u$, conditions (i) and (ii) and condition (33) imply that $\sigma^{\gamma}$ and $\nu^{\gamma}$ are bounded and Lipschitz continuous, and thus (37) admits a unique strong solution.

Then (39) is a simple application of Itô's formula, and shows that $V_{t}=u\left(t, S_{t}\right)$.

The existence of $\mathbb{P}$ is a direct application of Girsanov's theorem (see [22]), since $\sigma, \sigma^{-1}, \nu^{\gamma}$ are bounded. Letting again $\sigma^{\gamma}=\frac{\sigma}{1-\lambda \gamma}, \sigma^{\gamma}, 1 / \sigma^{\gamma}$ are bounded and one 
can take $\mathbb{P}$ absolutely continuous with respect to $\mathbb{P}_{0}, W^{\mathbb{P}}$ a $\mathbb{P}$-Brownian motion such that $d W^{\mathbb{P}}=d W^{\mathbb{P}_{0}}+\left(v^{\gamma} / \sigma^{\gamma}\right) d t$, and $S$ is also the strong solution to (34), (35), (36) under $\mathbb{P}$. (This solution is unique given the regularity assumed on $u$.)

Finally, (40) follows by taking the expectation of (39) under $\mathbb{P}$, having observed that

$$
\sigma^{\gamma}-\sigma=\sigma \frac{\lambda \gamma}{1-\lambda \gamma}
$$

REMARKS. The condition (ii) needs some a priori estimates to be enforced, but is trivially satisfied in the case where $\lambda$ is constant, which will be our main focus in this paper. Conversely, the condition on $\sigma$ is straightforward to check, and is also found to be necessary for the proof of uniform boundedness of the modified volatility (see Theorem 5.8).

This result shows that, under existence of a smooth solution $u$ to (24), the claim $\Phi$ is replicable by the self-financed strategy that consists in holding $\delta_{t}=\partial_{s} u\left(t, S_{t}\right)$ stocks, and that the value of this trading strategy is equal at any time to $u\left(t, S_{t}\right)$.

Note again that the profit generated by the hedging strategy is no more the usual expression $\int_{0}^{T} \partial_{S} u d S$, but includes an additional term due to the market impact (more exactly due to the difference of the price after market impact and the executed price, i.e., the liquidity costs). It is always positive (i.e., in favour of the option's seller). On the other hand, note that the change of volatility from $\sigma$ to $\sigma /(1-\lambda \gamma)$ acts always against the option's seller. As an immediate corollary of Theorem 4.1, the sum of the two impacts is always against the option's seller, that is, the price of the option with market impact is always greater than without market impact (see the remark after Theorem 4.1).

4. Dual formulation of the problem. We mention here the connection between our pricing equation and the dual formulation of second-order target problems studied in [35]. The equation we study here is still

$$
\begin{aligned}
\partial_{t} u+\frac{1}{2} \sigma^{2} F(\gamma) & =0, \\
F(\gamma) & =\frac{\gamma}{1-\lambda \gamma}, \\
u(T, s) & =\Phi(s),
\end{aligned}
$$

with $\gamma=s^{2} \partial_{s s} u$, and we assume that $\Phi, \sigma$ satisfy the conditions needed in Theorem 5.15 , so that $u$ is in $C^{1,3}\left([0, T] \times \mathbb{R}_{+}^{*}\right)$. We remark that $F$ is convex with respect to $\gamma$, and introduce $F_{\sigma}^{*}$ the Legendre transform of $\sigma^{2} F$

$$
\begin{aligned}
F_{\sigma}^{*}(a) & =\left(\sigma^{2} F\right)^{*}(a) \\
& =\sup _{\gamma}\left\{a \gamma-\sigma^{2} F(\gamma)\right\}= \begin{cases}\frac{1}{\lambda}\left(a^{1 / 2}-\sigma\right)^{2} & \text { if } a \geq 0, \\
+\infty & \text { otherwise. }\end{cases}
\end{aligned}
$$


Note that from the convexity of $F$, there will hold

$$
\sigma^{2} F(\gamma)=\sup _{a}\left\{a \gamma-F_{\sigma}^{*}(a)\right\}
$$

and that the supremum in (42) is reached for $a=\hat{a}=\left(\sigma^{\gamma}\right)^{2}=\frac{\sigma^{2}}{(1-\lambda \gamma)^{2}}$, which we recognize as the modified variance in our model. For $W^{\mathbb{P}}$ a $\mathbb{P}$-Brownian motion, with filtration $\left(\mathcal{F}_{t}\right)_{t \geq 0}$, we define

$$
\begin{aligned}
\mathcal{A}_{T}= & \left\{a_{t, t \in[0, T]}, a \text { is } \mathcal{F}_{t}\right. \text {-predictable, } \\
& \left.\exists \bar{a} \in R_{+}^{*} \text { such that } 0 \leq a_{t} \leq \bar{a} \mathbb{P} \text {-a.s. }\right\} .
\end{aligned}
$$

For $a \in \mathcal{A}_{T}$, we can define $S^{a, t, s}$ such that

$$
\begin{aligned}
S_{t}^{a, t, s} & =s, \\
d S_{r}^{a, t, s} & =S_{r}^{a, t, s}\left(a_{r}\right)^{1 / 2} d W_{r}^{\mathbb{P}}, \quad t \leq r \leq T,
\end{aligned}
$$

and define, for $\Phi$ a terminal condition,

$$
u^{a}(t, s)=\mathbb{E}^{\mathbb{P}}\left\{\Phi\left(S_{T}^{a, t, s}\right)-\int_{0}^{T} \frac{1}{2} F_{\sigma}^{*}\left(a_{u}\right) d u\right\} .
$$

Then applying Itô's formula to $u\left(r, S_{r}^{a, t, s}\right)$ (which is allowed from the regularity of $u$ ), we have that

$$
\begin{aligned}
\Phi\left(S_{T}^{a, t, s}\right)= & u(t, s)+\int_{t}^{T}\left(\partial_{t} u+\frac{1}{2} a_{r}\left(S_{r}^{a, t, s}\right)^{2} \partial_{s s} u\right) d r+\int_{t}^{T} \partial_{s} u a_{r}^{1 / 2} S_{r}^{a, t, s} d W_{r}^{\mathbb{P}} \\
= & u(t, s)+\int_{t}^{T}\left(\frac{1}{2} a_{r}\left(S_{r}^{a, t, s}\right)^{2} \partial_{s s} u-\frac{\sigma^{2}}{2} F\left(\left(S_{r}^{a, t, s}\right)^{2} \partial_{s s} u\right)\right) d r \\
& +\int_{t}^{T} \partial_{s} u a_{r}^{1 / 2} S_{r}^{a, t, s} d W_{r}^{\mathbb{P}}
\end{aligned}
$$

hence from (41),

$$
\Phi\left(S_{T}^{a, t, s}\right) \leq u(t, s)+\int_{t}^{T} \partial_{s} u\left(r, S_{r}^{a, t, s}\right) d S_{r}^{a, t, s}+\int_{t}^{T} \frac{1}{2} F_{\sigma}^{*}\left(a_{r}\right) d r,
$$

which yields

$$
\mathbb{E}^{\mathbb{P}}\left(\Phi\left(S_{T}^{a, t, s}\right)\right) \leq u(t, s)+\mathbb{E}^{\mathbb{P}}\left(\int_{0}^{T} \frac{1}{2} F_{\sigma}^{*}\left(a_{r}\right) d r\right) .
$$

This shows that $u^{a}(t, s) \leq u(t, s)$, with equality if and only if $a=\hat{a}=\sigma^{\gamma}$, hence when $S^{\hat{a}}$ is the risk-neutral diffusion (34), (35), (36). Letting, for $a \in \mathcal{A}_{T}$

$$
\mathcal{C}_{\sigma}^{t}(a)=\int_{t}^{T}\left(a_{t^{\prime}}^{1 / 2}-\sigma\left(t^{\prime}, s\right)\right)^{2} d t^{\prime}
$$

we have thus obtained the following: 
THEOREM 4.1. Let $u$ be a $C^{1,3}([0, T] \times(0,+\infty))$ smooth solution to $(24)$, (25), (26) satisfying (32), then

$$
u(t, s)=\sup _{a \in \mathcal{A}_{T}}\left\{\mathbb{E}^{\mathbb{P}}\left\{\Phi\left(S_{T}^{a, t, s}\right)-\frac{1}{2 \lambda} \mathcal{C}_{\sigma}^{t}(a)\right\}\right\},
$$

and the supremum is attained for $\hat{a}=\sigma^{\gamma}$ as in (35). In particular, this holds true if $\Phi, \sigma$ satisfy the assumptions to Theorem 5.15 for global regularity on $[0, T]$.

REMARKS. In Theorem 6.6, our result is stronger, as formula (48) holds for any $\Psi \leq \Phi$ such that $\left(1-\lambda s^{2} \partial_{s s} \Phi\right)(\Phi-\Psi)=0$, and holds in cases where $E^{\mathbb{P}}\left(\Phi\left(S_{T}\right)\right)$ might no be finite. We will also show that the optimal process $S_{t}$ remains a true martingale up to time $T$ under mild assumptions on $\Phi$, which allow for the optimal $\hat{a}$ to be unbounded, hence $\hat{a} \notin \mathcal{A}_{T}$.

As mentioned in Theorem 3.2, by taking $a=\sigma^{2}$ in (48), one obtains that

$$
u(t, s) \geq \mathbb{E}^{\mathbb{P}}\left(\Phi\left(S_{T}^{\sigma^{2}, t, s}\right)\right),
$$

and we recognize the right-hand side as the price of the claim $\Phi$ without market impact.

4.1. Relation to a problem of optimal transport. As mentioned in the Introduction, having found $u$ by solving (24), and specifying an initial distribution $\mathcal{L}_{0}$ for $S_{0}$, we let $\mathcal{L}_{T}^{\Phi}$ be the law of $S_{T}^{\hat{a}, 0, S_{0}}$. Then $\hat{a}=\frac{\sigma^{2}}{(1-\lambda \gamma)^{2}}$ realizes also

$$
\hat{a}=\operatorname{argmin}\left\{\mathbb{E}^{\mathbb{P}} \mathcal{C}_{\sigma}(a)\right\}
$$

where the infimum is taken over all the process $S^{a, 0, \mathcal{L}_{0}}, a \in \mathcal{A}_{T}$, that have laws $\mathcal{L}_{0}$ at time $0, \mathcal{L}_{T}^{\Phi}$ at time $T$, and follow (45). This problem of optimal transport by martingales has been studied by Touzi and Tan in [36]. Therefore, our regularity results can be seen as a step towards the regularity of optimal transport by diffusion, for this particular cost. Moreover, as we will see in Theorem 6.11, when the volatility is constant, we obtain an explicit formula to express the final density in terms of the terminal condition $\Phi$.

4.2. Interpretation in terms of robust hedging. The maximization problem (40) gives the optimal super-hedging price for the claim $\Phi$ over all possible diffusions that satisfy

$$
\mathbb{E}^{\mathbb{P}} \mathcal{C}_{\sigma}^{t}(a) \leq \mathbb{E}^{\mathbb{P}} \mathcal{C}_{\sigma}^{t}(\hat{a})
$$

The replication price is then equal to

$$
u(t, s)+\frac{1}{2 \lambda} \mathbb{E}^{\mathbb{P}} \mathcal{C}_{\sigma}^{t}(\hat{a})=\mathbb{E}^{\mathbb{P}}\left(\Phi\left(S_{T}^{t, s, \hat{a}}\right)\right) .
$$

Note also that (47) can be interpreted as the payoff of a volatility derivative, and that (48) gives the cheapest way to super-replicate the claim $\Phi$ by buying $\frac{1}{2 \lambda}$ units of the $\mathcal{C}_{\sigma}$ and delta-hedging, or alternatively, a lower bound on the price of the derivative $\mathcal{C}_{\sigma}$ given the price of the claim $\Phi$. 
5. Smooth solutions via Legendre-Fenchel transform. In this section, we prove existence and regularity of the solution to (24), (25), (26), in the cases of constant or local volatility $\sigma(t, s)$, and with constant market impact parameter $\lambda$. In particular, our result will give the conditions on $\sigma$ and $\Phi$ under which the solution $u$ has enough regularity to satisfy the assumptions of the verification theorem (Theorem 3.2), and to define properly the modified price evolution. The case of nonconstant $\lambda$ is treated in the Appendix.

\subsection{Notation.}

- For $A \subset[0, T], B \subset \mathbb{R}, C^{k+\alpha}(A \times B)$ will denote the usual Hölder space of functions with mixed derivatives of order up to $k$ being bounded and Hölder continuous with exponent $\alpha,\|\cdot\|_{C^{k+\alpha}(A \times B)}$ will then be the usual Hölder norm of order $k+\alpha$.

- For $k, m \in \mathbb{N}, 0<\alpha, \beta<1,\|u\|_{C_{s, t}^{k+\alpha, m+\beta}(A \times B)}$ (resp., $\|u\|_{C_{s}^{k+\alpha}(A \times B)}$ ) (resp., $\left.\|u\|_{C_{t}^{m+\beta}(A \times B)}\right)$ the Hölder norm of $u$ of order $k+\alpha$ with respect to $s$ and order $m+\beta$ with respect to $t$.

- We shall denote $C_{\mathrm{loc}}^{k+\alpha}(A \times B)$ the space of functions with bounded $C^{k+\alpha}(K)$ norm for all compact sets $K \subset A \times B$

- In a similar way, we define $C_{s, t, \text { loc }}^{k+\alpha, m+\beta}(A \times B)$.

- Will denote $C^{\infty}(A \times B)$ the space of infinitely differentiable functions on $A \times B$.

- Classically, we will denote $\mathbb{R}_{+}^{*}=(0,+\infty)$.

- Whenever needed, we will consider a Brownian motion $W_{t}^{\mathbb{P}}$ supported on $\left(\Omega, \mathbb{F}, \mathbb{P},\left(\mathcal{F}_{t}\right)_{t \geq 0}\right)$ a filtered probability space, and denote $\mathbb{E}^{\mathbb{P}}$ the expectation under the probability measure $\mathbb{P}$.

DEFinition 5.1. We shall say that $u$ is a classical solution to (24), (25), (26) if $u \in C_{s, t, \text { loc }}^{2,1}\left([0, T) \times \mathbb{R}_{+}^{*}\right) \cap C_{t, s}^{0,0}\left([0, T] \times \mathbb{R}_{+}^{*}\right)$, satisfies $(24)$ on $[0, T) \times \mathbb{R}_{+}^{*}$, (26) and satisfies (32) on $[0, T) \times \mathbb{R}_{+}^{*}$.

5.2. Some facts about the Legendre-Fenchel transform. A reference on this topic is [30].

DEFINITION 5.2. The Legendre transform of $u: \mathbb{R} \rightarrow \mathbb{R} \cup\{+\infty\}$ is defined by

$$
u^{*}(y)=\sup \{x y-u(x), x \in \mathbb{R}\}
$$

If $u$ is convex and lower semi-continuous (hereafter, 1.s.c.), then $\left(u^{*}\right)^{*}=u$. Moreover: 
- If $u$ is continuously differentiable at $x$ and $u^{*}$ is continuously differentiable at $\partial_{x} u(x)$, then

$$
\begin{aligned}
u^{*}\left(\partial_{x} u(x)\right)+u(x) & =\partial_{x} u(x) x, \\
\partial_{y} u^{*}\left(\partial_{x} u(x)\right) & =x,
\end{aligned}
$$

and the reverse equality hold since $\left(u^{*}\right)^{*}=u$.

- If $y \notin \overline{\partial_{x} u(\mathbb{R})}$, then $u^{*}(y)=+\infty$.

- At a point where $\partial_{x x} u(x)$ is defined and positive, $\partial_{y y} u^{*}\left(\partial_{x} u\right)$ is defined and satisfies

$$
\partial_{x x} u(x) \partial_{y y} u^{*}\left(\partial_{x} u\right)=1 .
$$

- If $u$ depends smoothly on a parameter $t$, for all $x \in \operatorname{Dom}(u)$ where $u$ is continuously differentiable at $x$,

$$
\partial_{t} u^{*}\left(t, \partial_{x} u\right)+\partial_{t} u(t, x)=0 .
$$

5.3. Transformation of the pricing equation via Legendre transforms. Starting from $u$ a classical solution of (24), (25), (26) (see Definition 5.1), we consider

$$
v= \begin{cases}-\lambda u(t, s)-\ln (s)-1 & \text { if } s>0, \\ +\infty & \text { otherwise }\end{cases}
$$

For $t \in[0, T), v$ is strictly convex under (32), and satisfies

$$
\partial_{t} v-\frac{\sigma^{2}(t, s)}{2} \frac{1}{s^{2} \partial_{s s} v}=-\frac{\sigma^{2}(t, s)}{2} .
$$

Consider $v^{*}$ the Legendre transform of $v$, from Definition 5.2, for the pair $(y(t, s), s(t, y))$ where $v(t, s)+v^{*}(t, y)=s y$, there will hold

$$
\begin{aligned}
y(t, s) & =\partial_{s} v(t, s), \\
s(t, y) & =\partial_{y} v^{*}(t, y), \\
\partial_{s s} v(t, s) \partial_{y y} v^{*}(t, y) & =1 .
\end{aligned}
$$

Moreover, one will have

$$
\begin{gathered}
v^{*}(t, y(t, s))=\ln (s)+\lambda\left(u-s \partial_{s} u\right), \\
\partial_{t} v(t, s)+\partial_{t} v^{*}(t, y(t, s))=0 .
\end{gathered}
$$

Then it follows that

$$
\begin{aligned}
\partial_{t} v^{*}+\frac{\sigma^{2}(t, s)}{2} \frac{\partial_{y y} v^{*}}{\left(\partial_{y} v^{*}\right)^{2}} & =\frac{\sigma^{2}(t, s)}{2}, \\
s & =\partial_{y} v^{*}(t, y) .
\end{aligned}
$$


By straightforward computations, $w$, the inverse function of $v^{*}$, satisfies

$$
\begin{aligned}
\partial_{t} w+\frac{\sigma^{2}(t, s)}{2}\left(\partial_{x x} w+\partial_{x} w\right) & =0 \\
s & =\frac{1}{\partial_{x} w(t, x)} .
\end{aligned}
$$

To construct properly $w$, we use also the Legendre transform. Since $v^{*}$ is increasing, a primitive of $v^{*}$ is convex, hence one will have for $W=\int v^{*}, \partial_{x} W^{*}\left(v^{*}(y)\right)=$ $y$ at any point where $v^{*}$ is continuous, that is, everywhere in the domain of $v^{*}$.

Construction of the terminal value for the transformed equations.

Proposition 5.3. Let $\Phi: \mathbb{R}_{+}^{*} \rightarrow \mathbb{R}$ satisfy $\lambda s^{2} \partial_{s s} \Phi \leq 1$. Define $v_{T}, v_{T}^{*}, w_{T}$ such that

$$
\begin{aligned}
v_{T} & = \begin{cases}-\ln (s)-\lambda \Phi-1 & \text { if } s>0, \\
+\infty & \text { otherwise, }\end{cases} \\
v_{T}^{*} & =\left(v_{T}\right)^{*}, \\
w_{T} & =\left[v_{T}^{*}\right]^{-1} \quad \text { defined as } w_{T}=\partial_{x}\left(\int v_{T}^{*}\right)^{*}, \\
V_{T}(s) & =v_{T}^{*}\left(\partial_{s} v_{T}\right), \\
\mathcal{S}_{T} & =\partial_{y} v_{T}^{*}\left(w_{T}\right)=\frac{1}{\partial_{x} w_{T}} .
\end{aligned}
$$

Then:

1. $v_{T}^{*}$ is nondecreasing.

2. $v_{T}^{*}\left(\partial_{s} v_{T}\right)=\ln (s)+\lambda\left(\Phi-s \partial_{s} \Phi\right)$ wherever $\partial_{s} v_{T}$ exists.

3. $\lim _{y \rightarrow-\infty} \partial_{y} v^{*}=0$.

4. Either $\exists L \in \mathbb{R}$ such that $v^{*} \equiv+\infty$ above $L$ or $\lim _{y \rightarrow+\infty} \partial_{y} v^{*}=+\infty$.

5. If $\lim _{s \rightarrow 0} v_{T}$ is finite, then $w_{T}$ is identically $-\infty$ below this limit, otherwise $w_{T}$ is finite everywhere and $\lim _{x \rightarrow-\infty} \partial_{x} w_{T}=+\infty$.

6. If $\partial_{S} v_{T}$ is constant on some interval $(C,+\infty)$, then $\partial_{x} w_{T}$ is identically 0 above $C^{\prime}$ for some constant $C^{\prime}$; otherwise, $\partial_{x} w_{T}$ is positive everywhere and $\lim _{x \rightarrow+\infty} \partial_{x} w_{T}=0$.

7. For all $s$ where $\lambda s^{2} \partial_{s s} \Phi<1$ and $\partial_{s} \Phi$ is continuous, there holds

$$
\mathcal{S}_{T}\left(v_{T}^{*}\left(\partial_{s} v_{T}(s)\right)\right)=\mathcal{S}_{T}\left(\ln (s)+\lambda\left(\Phi-s \partial_{s} \Phi\right)\right)=s .
$$

8. If $w$ is finite everywhere on $\mathbb{R}$, one has necessarily that

$$
\begin{aligned}
\lim _{s \rightarrow 0} v_{T} & =+\infty, \\
\lim _{y \rightarrow-\infty} v_{T}^{*} & =-\infty .
\end{aligned}
$$


9. From points 2 and 7, we thus have Lebesgue a.e.

$$
\begin{aligned}
V_{T}(s) & =\ln (s)+\lambda\left(\Phi-s \partial_{s} \Phi\right), \\
\mathcal{S}_{T}\left(V_{T}(s)\right) & =s .
\end{aligned}
$$

10. Let $u$ be a classical solution to (24), (25), then one can define $v(t, \cdot)$, $v^{*}(t, \cdot), w(t, \cdot), V(t \cdot), \mathcal{S}(t, \cdot)$ from $u(t, \cdot)$ following the same procedure, and the above properties will apply.

11. $v, v^{*}, w$ will follow respectively (51), (52), (53), and there will hold for $s>0, t \in[0, T)$,

$$
\mathcal{S}\left(t, \ln (s)+\lambda\left(u-s \partial_{s} u\right)\right)=s .
$$

PRoof. 1. The first point comes from the fact that $v_{T}=+\infty$ for $s<0$.

2. This is just the definition of the Legendre transform, and the fact that $y=$ $\partial_{s} v_{T}$ for the optimal $s$ (see Definition 5.2).

3. The third point comes the fact that $v$ is defined and finite on $(0,+\infty)$. If one had $\lim _{y \rightarrow-\infty} \partial_{y} v^{*}=l>0$, then this would imply that $v \equiv+\infty$ below $l$.

4. The two cases correspond to $\lim _{s \rightarrow+\infty} \partial_{s} v$ being either finite (equal to $L$ ) or $+\infty$. ( $v$ being convex, one of the two must hold.)

5. Notice that

$$
v_{T}=+\infty \quad \text { for } s<0 \quad \Longrightarrow \quad \lim _{y \rightarrow-\infty} v_{T}^{*}=-\lim _{s \rightarrow 0} v_{T} .
$$

If $\lim _{s \rightarrow 0} v_{T}(s)$ is finite, then $\lim _{y \rightarrow-\infty} v^{*}=-\lim _{s \rightarrow 0} v_{T}(s)$. This in turn implies that $w_{T}=-\infty$ below this limit. The other assertion follows from point 3 since $\partial_{x} w_{T}=\frac{1}{\partial_{y} v^{*}}$.

6. This corresponds to the two cases of point 4 . If $\partial_{s} v \equiv l$ for $s \geq s_{0}$, then

$$
\begin{aligned}
\lim _{y \rightarrow l} \partial_{y} v_{T}^{*} & =s_{0}, \\
v_{T}^{*}(y) & =+\infty \quad \text { for } y>l, \\
w_{T}(x) & =l \quad \text { for } x \geq v_{T}^{*}(l) .
\end{aligned}
$$

Otherwise, we have $\lim _{y \rightarrow+\infty} \partial_{y} v^{*}=+\infty$. Then $\left[w_{T}\right]^{-1}=v^{*}$ implies

$$
\partial_{x} w_{T}\left(v_{T}^{*}(y)\right)=\frac{1}{\partial_{y} v_{T}^{*}}(y),
$$

and the result follows. 7. Point 7 follows directly from the definition of $\mathcal{S}_{T}$ and (57).

8. For point 8 , the first part follows from point 5 , the second part comes again from (56).

9. Point 9 is straightforward since $u$ satisfies (32).

10. Points 10 and 11 follow from direct computations performed above. 
5.4. Main assumptions. Throughout the paper, we will assume the following.

ASSUMPTION 5.4. There exist positive constants $\bar{\sigma}, \underline{\sigma}$ such that

$$
\forall t \in[0, T], s>0, \quad \underline{\sigma} \leq \sigma(t, s) \leq \bar{\sigma} .
$$

ASSUMPTION 5.5. $\Phi$ satisfies (31), and letting $\mathcal{S}_{T}$ be defined from $\Phi$ through (54), (55), there exists $\bar{\sigma}$ as in Assumption 5.4, $\varepsilon>0$, such that

$$
\int_{\mathbb{R}} \exp \left(-\frac{x^{2}}{2 \bar{\sigma}^{2}(T+\varepsilon)}\right) \frac{1}{\mathcal{S}_{T}(x)} d x \text { is finite. }
$$

5.5. The case of constant volatility. We now show that (24), (25), (26) admits a unique smooth solution when $\sigma, \lambda$ are constant. In this case, $\partial_{x} w$ solves also (53), hence one can recover the function $\partial_{x} w:\left(\ln (s)+\lambda\left(u-s \partial_{s} u\right) \rightarrow \frac{1}{s}\right)$ by solving a simple heat equation, which then leads to the solution $u$. Therefore, the condition for existence of a smooth solution can be stated as a condition on the function $\mathcal{S}_{T}: v^{*} \rightarrow s$ at time $T$. We will have the following result.

THEOREM 5.6. Let $\Phi, \sigma$ satisfy Assumptions 5.4 and 5.5:

(i) There exists a unique u classical solution to (24), (25), (26). It belongs to $C^{\infty}\left([0, T) \times \mathbb{R}_{+}^{*}\right)$.

(ii) For all $(t, s) \in[0, T] \times \mathbb{R}_{+}^{*}$,

$$
\inf _{s}\left\{s^{2} \partial_{s s} \Phi\right\} \leq s^{2} \partial_{s s} u(t, s) \leq \sup _{s}\left\{s^{2} \partial_{s s} \Phi\right\} .
$$

(iii) If for $k \geq 2, v>0, \Phi \in C^{k}\left(\mathbb{R}_{+}^{*}\right)$ and $\lambda s^{2} \partial_{s s} \Phi \leq 1-v$, then

$$
\left|s^{k} \partial_{s}^{k} u\right|_{L^{\infty}\left([0, T] \times \mathbb{R}_{+}^{*}\right)} \leq C_{k}\left(\left|s^{k^{\prime}} \partial_{s}^{k^{\prime}} \Phi\right|_{L^{\infty}\left(\mathbb{R}_{+}^{*}\right)}, k^{\prime} \leq k\right) .
$$

REMARK. As we will see, $\mathcal{S}_{T}$ is positive and increasing, thus Assumption 5.5 on $\Phi$ is only about the behaviour of $\Phi$ near 0 . It allows the set $\left\{1-\lambda s^{2} \partial_{s s} \Phi=0\right\}$ to be nonempty, in particular, any globally Lipschitz function $\Phi$ such that

$$
\lambda s^{2} \partial_{s s} \Phi \leq 1
$$

satisfies Assumption 5.5. Surprisingly, even if $\lambda s^{2} \partial_{s s} \Phi \equiv 1$ for $s$ above a certain threshold, Assumption 5.5 might be satisfied, as it just implies that $\frac{1}{\mathcal{S}_{T}}$ is identically 0 above a certain threshold. Conversely, if $\lambda s^{2} \partial_{s s} \Phi \equiv 1$ for $s$ close to 0 then Assumption 5.5 cannot be satisfied. This is a feature of the log-normal dynamics that "send the mass to 0". 
Proof OF TheOREM 5.6. Starting from the terminal payoff $\Phi$, as explained above, one constructs $v_{T}$, and then $v_{T}^{*}, w_{T}$ and then $\frac{1}{\mathcal{S}_{T}}=\partial_{x} w_{T}$ which is defined almost everywhere on the set $\partial_{y} v_{T}^{*}>0$. For $t<T$, let $w$ be given by

$$
w(t, x)=\frac{1}{\sigma(2 \pi(T-t))^{1 / 2}} \int_{\mathbb{R}} \exp \left(-\frac{\left(x+\frac{\sigma^{2}}{2}(T-t)-z\right)^{2}}{2 \sigma^{2}(T-t)}\right) w_{T}(z) d z .
$$

Assumption 5.5 implies that $w$ is well defined, belongs to $C_{\mathrm{loc}}^{\infty}([0, T) \times \mathbb{R})$ and solves (53) (which is a heat equation with constant coefficients) and, therefore, $\partial_{x} w$ solves also (53). The properties listed in Proposition 5.3 ensure that, for $t<T$, $w$ is strictly increasing and strictly concave, $\partial_{x} w$ being given by

$$
\partial_{x} w(t, x) \frac{1}{\sigma(2 \pi(T-t))^{1 / 2}} \int_{\mathbb{R}} \exp \left(-\frac{\left(x+\frac{\sigma^{2}}{2}(T-t)-z\right)^{2}}{2 \sigma^{2}(T-t)}\right) \frac{1}{\mathcal{S}_{T}(z)} d z,
$$

and having limits $+\infty$ at $-\infty$ and 0 at $+\infty$.

Then one follows backward our previous transformations of $u$ :

- The inverse of $w$ (in the sense of inverse functions) will satisfy (52),

- its Legendre transform $\left(w^{-1}\right)^{*}$ will then satisfy (51),

- finally $u(t, s)=-\lambda^{-1}\left(\left(w^{-1}\right)^{*}(t, s)+\ln (s)+1\right)$ will satisfy (24), (25), (26).

The bounds on $s^{2} \partial_{s s} u$ are a direct consequence of the following lemma.

LeMma 5.7. Let $u$ be the classical solution to (24) on $[0, T)$ with constant $\sigma$, constructed as above from $w$. Then $\sup _{s>0}\{\gamma(t, s)\}$ is nondecreasing and $\inf _{s>0}\{\gamma(t, s)\}$ is nonincreasing.

Before proving this lemma, we complete the proof of Theorem 5.6. The bounds on $s^{k} \partial_{s}^{k} u$ can be obtained by looking at $v(t, y)=u\left(t, e^{y}\right)$ that solves

$$
\partial_{t} v+\frac{\sigma^{2}}{2} \frac{\partial_{y y} v-\partial_{y} v}{1-\lambda\left(\partial_{y y} v-\partial_{y} v\right)}=0 .
$$

The bounds on $\partial_{y}^{k} v$ are classically obtained by differentiating the equation, and imply the bounds on $s^{k} \partial_{s}^{k} u$.

Finally, the uniqueness is a consequence of Widder's theorem (see [22], Chapter 4, Theorem 3.6) since starting from $u$ a classical solution to (24) one can build $w$ from $u$ as explained above, and then $\partial_{x} w$ will be a positive solution to the heat equation, for which uniqueness holds.

Proof of LemMA 5.7. Observe that for $u$ a classical solution of (24), and $w$ defined from $u$, one has $\partial_{x} w=\frac{1}{\mathcal{S}}$ and $\partial_{x x} w=-\frac{1}{\mathcal{S}(1-\lambda \gamma)}$. We will have proved the lemma if we show that for any $C>0$, if $C \partial_{x} w_{T}+\partial_{x x} w_{T} \geq 0$ (resp., $\leq 0$ ) then for time $t<T$ the same inequality holds. This will hold if $C \partial_{x} w_{T}+\partial_{x x} w_{T}$ 
which solves the heat equation, and satisfies the maximum principle. This type of result is in general is not true on the whole line without any growth assumptions (see [38]), but in our case this is granted as we have constructed $w$ through the representation formula (59), therefore, $C \partial_{x} w_{T}+\partial_{x x} w_{T}$ can be obtained through a similar representation formula, and the result follows.

5.6. The case of nonconstant volatility. Here, we show the following a priori estimate.

THEOREM 5.8. Assume that there exist constants $M, A, B, \varepsilon_{1}, \varepsilon_{2}, C_{\sigma}, \underline{\sigma}, \bar{\sigma}$ with $M, \varepsilon_{1}, \varepsilon_{2}, \underline{\sigma}, \bar{\sigma}$ positive, such that, for $\Lambda=\lambda^{-1}$ :

(i) For $s \leq 1 / M$,

$$
\left(\varepsilon_{1}-\Lambda\right) \ln (s)-A+B s \leq \Phi(s) \leq\left(\varepsilon_{1}-\Lambda\right) \ln (s)+A+B s .
$$

(ii) For $s \geq M$,

$$
\left(\varepsilon_{2}-\Lambda\right) \ln (s)-A+B s \leq \Phi(s) \leq\left(\varepsilon_{2}-\Lambda\right) \ln (s)+A+B s .
$$

(iii) $\underline{\sigma} \leq \sigma(t, s) \leq \bar{\sigma}$ (Assumption 5.4).

(iv) $\left|s \partial_{s} \sigma\right|+\left|s^{2} \partial_{s s} \sigma\right|+\left|\partial_{t} \sigma\right|+\left|s \partial_{s t} \sigma\right| \leq C_{\sigma}$.

Let $u$ be a classical solution to (24), (25), (26), then for all $\tau>0$ small there exists $C_{\tau}\left(M, A, B, \varepsilon_{1}, \varepsilon_{2}, C_{\sigma}, \underline{\sigma}, \bar{\sigma}\right)$ such that for $0 \leq t \leq T-\tau, \forall s>0$,

$$
\begin{aligned}
-C_{\tau} & \leq s^{2} \partial_{s s} u(t, s) \leq \Lambda-1 / C_{\tau}, \\
\left|s^{3} \partial_{s}^{3} u\right| & \leq C_{\tau} .
\end{aligned}
$$

If, moreover

(v) $\Phi \in C^{3+\alpha}, s^{3} \partial_{s}^{3} \Phi$ is bounded and $\Phi$ satisfies (33),

then $u$ satisfies $(60),(61)$ on $[0, T] \times \mathbb{R}_{+}^{*}$ (in other words, $C_{\tau}$ remains bounded as $\tau \rightarrow 0)$.

REMARKS. A useful consequence of this result is to be able to define the dynamics of $S_{t}$ up to time $T-\tau$ for all $\tau>0$ without relying on stopping times. For this, we need a uniform bound like (60).

The conditions on $\Phi$ might not be minimal, but they allow for a large class of payoffs: all payoffs with linear or logarithmic growth at 0 and infinity. In particular, the conditions are satisfied by any finite combination of vanilla options.

If $\varepsilon_{1}=\varepsilon_{2}$, the proof becomes much simpler. The interest of the result lies in the fact that one can prescribe independently the behaviour at 0 and $+\infty$.

Preliminary results. We will treat (52) as an equation of the general form

$$
\partial_{t} v^{*}+A\left(t, \partial_{y} v^{*}\right) \partial_{y y} v^{*}+B\left(t, \partial_{y} v^{*}\right)=0 .
$$

The strategy will be the following: 
- For equation (62), there exist (see [25]) local regularity results that yield $C^{1, \alpha}$ regularity in space conditional to uniform ellipticity of $A$ and Lipschitz a priori estimates on $v^{*}$.

- In order to have those a priori estimates, we will use a barrier argument: we will show by the comparison principle that the solution is pinched between an upper and a lower bound, and this control used with the convexity of the solution will in turn lead to a control of the gradient. This barrier argument is the most delicate part of the proof, it is stated in Proposition 5.10.

- The Hölder regularity of $\partial_{y} v^{*}$ will then imply that (52) can be looked at as a linear uniformly parabolic equation, with Hölder continuous coefficients, and this in turn through Schauder estimates (see [25] again) leads to $C_{t, y}^{1+\alpha / 2,2+\alpha}$ regularity.

- The growth conditions (i) and (ii) will then allow by a scaling argument to show the uniform bound (60).

Note that from our assumption $\lim _{s \rightarrow+\infty} \partial_{s} \Phi(s)=B$, hence $v^{*}(T, y) \equiv+\infty$ for $y>-\lambda B$, and the equation (52) has a singular boundary condition which will need careful treatment. On the other hand, from points 3 and 8 of Proposition 5.3, $\lim _{y \rightarrow-\infty} \partial_{y} v^{*}(t, y)=0$ and $\lim _{y \rightarrow-\infty} v^{*}(t, y)=-\infty$. One should think of $v^{*}$ as a perturbation of $y \rightarrow-\ln (-\lambda B-y)$; see Figure 2 .

The barrier argument and the gradient estimates that result thereof is given in the following Proposition 5.10, whose proof will be given in Appendix A. Aside from analytical technicalities, the difficulty here comes from the fact that there is a priori no comparison theorem for solutions to (24), (25), (26). Indeed, this is a nonlinear parabolic equation, in an unbounded domain, and we consider unbounded solutions, therefore, one cannot expect a comparison result to hold in full

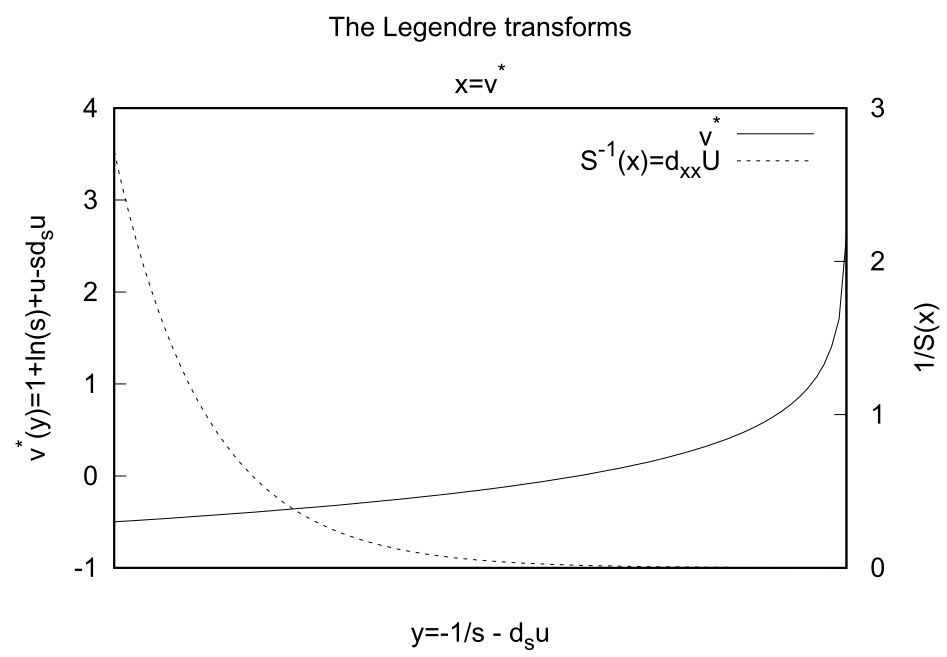

FIG. 2. $\quad v^{*}$ and $\mathcal{S}_{T}$ for $\Phi(s)=s-\frac{1}{2} \ln (s), \lambda=1$. 
generality; see Lemma A.1 in the Appendix. A useful corollary of this result is given in Proposition 5.9 hereafter (whose proof is also deferred to Appendix A).

Proposition 5.9. Let $\Phi, \sigma$ satisfy Assumptions 5.4 and 5.5. Let $u$ be a classical solution to (24), (25), (26). Let $\bar{u}$ (resp., $\underline{u}$ ) be the solution to (24), (25), (26) with $\sigma=\bar{\sigma}$ (resp., $\sigma=\underline{\sigma}$ ). Then

$$
\underline{u}-(T-t) \frac{\bar{\sigma}^{2}-\underline{\sigma}^{2}}{2} \leq u \leq \bar{u}+(T-t) \frac{\bar{\sigma}^{2}-\underline{\sigma}^{2}}{2} .
$$

PROPOSITION 5.10. Under the assumptions of Theorem 5.8,

(i) One can find another constant $A^{\prime}$ instead of $A$ such that the properties (i) and (ii) of Theorem 5.8 are satisfied by the solution $u$ on $[0, T]$.

(ii) There exist $\theta_{1}, \theta_{2}>0$ that depend on $A, B, \varepsilon_{1}, \varepsilon_{2}, M$ in Theorem 5.8 such that for $t \in[0, T], y<-\lambda B$,

$$
\frac{\theta_{1}}{-(y+\lambda B)} \leq \partial_{y} v^{*} \leq \frac{\theta_{2}}{-(y+\lambda B)} .
$$

(iii) There exists $\theta=\theta\left(\theta_{2}, \theta_{1}\right)>1$ such that $\forall \alpha>0, t \leq T$,

$$
\frac{1}{\partial_{y} v^{*}(-\lambda B-\alpha)}+\alpha \leq \frac{1}{\partial_{y} v^{*}(-\lambda B-\theta \alpha)} \text {. }
$$

PROOF OF THEOREM 5.8. We can invoke an appropriate result of regularity (see Lieberman [25], Lemma 12.13), stating that $\partial_{y} v^{*}$ is locally Hölder continuous in space. Adapted to our case here is the result.

LEMMA 5.11 ([25]). Let $v^{*}$ solve on $(a, b) \times[0, T]$ equation $(52)$, such that for some $v>0, v \leq \partial_{y} v^{*} \leq v^{-1}$. Then for $\tau>0$ small, $\beta \in(0,1)$, for $t \in[0, T-\tau]$, for $\omega \subset \subset(a, b)$,

$$
\left\|\partial_{y} v^{*}\right\|_{C^{\beta, \beta / 2}(\omega \times[0, T-\tau])} \leq C(\beta, v, \tau, \sigma, b-a, \omega) .
$$

The dependence with respect to $\sigma$ is controlled by $\underline{\sigma}, \bar{\sigma}$ in Assumption 5.4, and $\sup _{[0, T] \times(a, b)}\left|\partial_{s} \sigma(t, s)\right|+\left|\partial_{t} \sigma(t, s)\right|$. If moreover $v^{*}(t=T) \in C_{1 \mathrm{loc}}^{1+\beta}(a, b)$, then $v^{*} \in C_{s, \text { loc }}^{1+\beta}([0, T] \times(a, b))$.

We go on with the proof of Theorem 65, and first prove (60). Let $\theta$ be as in Proposition 5.10, for $y+\lambda B \in[-\theta,-1]$, consider for some $\alpha>0$,

$$
u^{\alpha}(t, y)=v^{*}(t,-\lambda B+\alpha(y+\lambda B)) .
$$

Then $\partial_{y} u^{\alpha}(t, y)=\alpha \partial_{y} v(t,-\lambda B+\alpha(y+\lambda B))$, and $u^{\alpha}$ solves

$$
\partial_{t} u^{\alpha}+\frac{\sigma^{2}\left(t, \alpha^{-1} \partial_{y} u^{\alpha}\right)}{2} \frac{\partial_{y y} u^{\alpha}}{\left(\partial_{y} u^{\alpha}\right)^{2}}=\frac{\sigma^{2}\left(t, \alpha^{-1} \partial_{y} u^{\alpha}\right)}{2} .
$$


We now use Proposition 5.10, point (ii), which yields that for some positive constants $\theta_{1}^{\prime}, \theta_{2}^{\prime}$, one has

$$
y+\lambda B \in[-\theta,-1] \Rightarrow \theta_{1}^{\prime} \leq \partial_{y} u^{\alpha} \leq \theta_{2}^{\prime} .
$$

The assumptions of Theorem 5.8 imply a uniform bound on $s \partial_{s} \sigma(t, s)$, which implies a bound on $\partial_{z}\left(\sigma^{2}\left(t, \alpha^{-1} z\right)\right)$, uniformly with respect to $\alpha>0, t \in$ $[0, T], z \in\left[\theta_{1}^{\prime}, \theta_{2}^{\prime}\right]$.

Hence, Lemma 5.11 applies to equation (64), and implies that $\partial_{y} u^{\alpha}$ is bounded and Hölder continuous uniformly with respect to $\alpha$.

Using Schauder estimates ([25], see also [41] for a very synthetic proof) this gives a uniform control on $\partial_{y y} u^{\alpha}$ and $\partial_{t} u^{\alpha}$ in $C_{s}^{\beta}([0, T-\tau] \times[-\theta-\lambda B$, $-1-\lambda B])$. This in turn guarantees that $\partial_{t} v^{*}$ is also uniformly bounded in $[0, T-\tau] \times \mathbb{R}_{+}^{*}$, and and since $\partial_{t} u(t, s)=-\partial_{t} v^{*}(t, y)$, we obtain that $\frac{1}{1-\lambda s^{2} \partial_{s s} u}$ is uniformly bounded on $[0, T-\tau] \times \mathbb{R}_{+}^{*}$.

We obtain a bound from below on $s^{2} \partial_{s s} u$ as a consequence of Harnack inequality. Using the choice of $\theta$ from Proposition 5.10 and (63), $u^{\alpha}$ will satisfy uniformly with respect to $\alpha$

$$
\frac{1}{\partial_{y} u^{\alpha}}(-\lambda B-1)+1 \leq \frac{1}{\partial_{y} u^{\alpha}}(-\lambda B-\theta),
$$

and given the bound on $\partial_{y} u^{\alpha}$, this implies

$$
\partial_{y} u^{\alpha}(-\lambda B-1) \geq \partial_{y} u^{\alpha}(-\lambda B-\theta)+\varepsilon_{0}
$$

for some $\varepsilon_{0}>0$. Then, differentiating twice the equation (64), we obtain that $z=$ $\partial_{y y} u^{\alpha}$ solves an equation of the form

$$
\partial_{t} z+\partial_{y}\left(A\left(t, \partial_{y} u^{\alpha}\right) \partial_{y} z\right)+\partial_{y}\left(C\left(t, \partial_{y} u^{\alpha}, z\right) z\right)=0
$$

where $A=\frac{\sigma^{2}\left(t, \alpha^{-1} \partial_{y} u^{\alpha}\right)}{2\left(\partial_{y} u^{\alpha}\right)^{2}}$. Under the assumption that $s \partial_{s} \sigma$ is bounded, the coefficient $A, C$ are uniformly bounded with respect to $\alpha$, and $A$ is also bounded away from 0 . We already now that $z$ is positive, bounded and continuous, moreover by (65) we have that

$$
\sup \left\{\partial_{y y} u^{\alpha}(t, y), y \in[-\lambda B-\theta,-\lambda B-1]\right\} \geq \frac{\varepsilon_{0}}{\theta-1} .
$$

We can now invoke the Harnack inequality for the solution of (66) (see [25], Theorem 6.27) which implies that, locally, the supremum of $w$ at a given time $t$ is controlled by the infimum of $w$ at time $t^{\prime}<t$. Hence the infimum of $w$ has to stay uniformly away from 0 . This in turn implies that $\frac{\partial_{y y} v^{*}}{\left(\partial_{y} v^{*}\right)^{2}}$ remains uniformly bounded away from 0 on $[0, T-\tau]$, hence that $s^{2} \partial_{s s} u$ is bounded away from $-\infty$, which achieves the proof of $(60)$.

To prove (61), we use again (66). Since $s^{2} \partial_{s s} \sigma$ is bounded, we also have that $y \rightarrow\left(\sigma\left(t, \alpha^{-1} \partial_{y} u^{\alpha}\right)\right)$ is bounded in $C_{s}^{1+\beta}(K)$ for $K$ compactly supported in 
$[0, T) \times(-\infty,-\lambda B)$. We also assume that $s \partial_{s t} \sigma$ bounded. Standard parabolic regularity applied to (66) then yield that $u^{\alpha}$ belongs to $C_{s}^{3+\beta}([0, T-\tau] \times[-\lambda B-\theta$, $-\lambda B-1]$ ), with bounds uniform with respect to $\alpha$. Then, by considering $u-B s$ instead of $u$, we can assume without loss of generality that $B=0$. In this case,

$$
\begin{aligned}
u^{\alpha}(t, y) & =v^{*}(t, \alpha y), \\
v(t, s) & =\left(u^{\alpha}(t)\right)^{*}(s / \alpha),
\end{aligned}
$$

hence

$$
\begin{aligned}
\alpha^{2} \partial_{s s} v(\alpha s) & =\frac{1}{\partial_{y y} u^{\alpha}\left(\alpha \partial_{s} v(\alpha s)\right)}, \\
\alpha^{3} \partial_{s}^{3} v(\alpha s) & =\frac{-\partial_{y}^{3} u^{\alpha} \alpha^{2} \partial_{s s} v(\alpha s)}{\left(\partial_{y y} u^{\alpha}\left(\alpha \partial_{s} v(\alpha s)\right)\right)^{2}} .
\end{aligned}
$$

From Proposition 5.10, for fixed $s, \alpha \partial_{s} v(\alpha s)$ is bounded with respect to $\alpha$, and from the proof of (60), $\partial_{y y} u^{\alpha}, 1 / \partial_{y y} u^{\alpha}$ are bounded on any compact set. This implies that $s^{2} \partial_{s s} v$ and $s^{3} \partial_{s}^{3} v$ are bounded, and the same holds then for $u$.

To prove regularity up to the initial boundary when the initial data is such that $u^{\alpha}(T)$ is uniformly bounded in $C^{2+\alpha}$ (which is the case if $s^{3} \partial_{s}^{3} \Phi$ is bounded), we use the last part of Lemma 5.11 that yields $C_{s}^{1+\beta}$ bounds up to time $T$, proceed in a similar manner, and apply standard parabolic regularity up to the boundary (see [25], Theorem 5.14). This achieves the proof of Theorem 5.8.

5.7. Local interior bounds. Under the minimal Assumptions 5.4, 5.5, we can still establish local interior regularity. The proof of this result is deferred to Appendix B, as it is a similar to the proof of Theorem 5.8.

THEOREM 5.12. Let $\sigma, \Phi$ satisfy Assumptions 5.4, 5.5, with $\sigma \in$ $C_{\mathrm{loc}}^{1+\alpha}\left([0, T] \times \mathbb{R}_{+}^{*}\right)$. Then, any classical solution $u$ to $(24),(25)$, (26) satisfies: For all compact set $K \subset[0, T) \times \mathbb{R}_{+}^{*}, \alpha \in(0,1)$,

$$
\begin{gathered}
\|u\|_{C_{s, t}^{3+\alpha, \frac{3}{2}+\alpha / 2}(K)} \leq Q, \\
-Q \leq s^{2} \partial_{s s} u \leq 1 / \lambda-1 / Q, \\
Q=Q\left(K, \Phi, \underline{\sigma}, \bar{\sigma},\|\sigma\|_{C^{1+\alpha}(K)}, \alpha\right) .
\end{gathered}
$$

If $\sigma$ is only $C_{\mathrm{loc}}^{\alpha}\left([0, T] \times \mathbb{R}_{+}^{*}\right)$, then $u$ is locally bounded in $C_{s, t, 1 \mathrm{loc}}^{2+\alpha / 1+\alpha / 2}$ with similar bounds.

REMARK. The bound $Q$ can be be made dependent only on $\underline{\Phi}, \bar{\Phi}$ for $\Phi$ varying in $\left\{\Phi: \lambda s^{2} \partial_{s S} \Phi \leq 1, \underline{\Phi} \leq \Phi \leq \bar{\Phi}\right\}$.

In view of Theorem 5.6, the condition on $\Phi$ is quite sharp: when $\sigma \equiv \bar{\sigma}$ and Assumption 5.5 does not hold, the solution $u$ can not be finite for any $t<0$. 
5.8. Initial regularity. We also mention that local (in space) regularity holds up to time $T$ whenever $\Phi$ is locally smooth and satisfies locally (32).

THEOREM 5.13. In addition to the assumptions of Theorem 5.12, if $\Phi \in$ $C^{2+\alpha}([a, b])$ for some $a<b$ where it satisfies (32), then

$$
u \in C_{s, t, \operatorname{loc}}^{2+\alpha, 1+\alpha / 2}([0, T] \times(a, b)) .
$$

If moreover $\Phi \in C^{3+\alpha}([a, b])$, then $u \in C^{3+\alpha, \frac{3+\alpha}{2}}((a, b) \times[0, T])$. For $\left[a^{\prime}, b^{\prime}\right] \subset$ $(a, b)$, the bounds on $\|u\|_{C_{s, t}^{2+\alpha, 1+\alpha / 2}\left([0, T] \times\left[a^{\prime}, b^{\prime}\right]\right)}\left(\right.$ resp., $\left.\|u\|_{C_{s, t}^{3+\alpha, \frac{3+\alpha}{2}}\left([0, T] \times\left[a^{\prime}, b^{\prime}\right]\right)}\right)$ depend on the same quantities as in Theorem 5.12 and on $\|\Phi\|_{C^{2+\alpha}([a, b])}$ (resp., $\left.\|\Phi\|_{C^{3+\alpha}([a, b])}\right)$.

PROOF. It uses a classical cutoff argument. Multiplying $v^{*}$ by a cutoff function $\eta(y)$ compactly supported in $(a, b), h=v^{*} \eta$ solves

$$
\partial_{t} h+A \partial_{y y} h+B=A\left(\partial_{y} v^{*} \partial_{y} \eta+v^{*} \partial_{y y} \eta\right)
$$

for $A=A\left(t, \partial_{y} v^{*}\right), B=B\left(t, \partial_{y} v^{*}\right), A, A^{-1}, B$ bounded. We already have from Lemma 5.11 a global $C_{s, t, \text { loc }}^{\alpha, \alpha / 2}([0, T] \times(a, b))$ bound on $\partial_{x} v^{*}$. Hence, $A, B$ are Hölder continuous. Since $h$ is $C_{s}^{2+\alpha}$ smooth on the parabolic boundary of $[a, b] \times$ $[0, T]$, classical Schauder regularity (see again Lieberman [25], Theorem 5.14) applies up to the boundary and yields the desired result.

5.9. Construction of solutions. We now prove the existence of solutions, using the a priori bounds and the continuity method:

Proposition 5.14. Let $\Phi, \sigma$ satisfy the assumptions (i) to (v) of Theorem 5.8. Then there exists a classical solution to (24), (25), (26).

PROOF. We consider for $\varepsilon \in[0,1] \sigma^{\varepsilon}$ defined as follows:

$$
\sigma^{\varepsilon}= \begin{cases}1 & \text { if } t \geq T(1-\varepsilon), \\ ((1-\varepsilon) \sigma(t, s)+\varepsilon) & \text { if } t \leq T(1-2 \varepsilon), \\ 1+\varphi^{\varepsilon}(t)(1-\varepsilon)(\sigma(t, s)-1) & \text { if } t \in[T(1-2 \varepsilon), T(1-\varepsilon)],\end{cases}
$$

where $\varphi^{\varepsilon} \in C^{\infty}(\mathbb{R})$ is nonnegative, nonincreasing and satisfies:

$$
\varphi^{\varepsilon}= \begin{cases}0 & \text { if } t \geq T(1-\varepsilon), \\ 1 & \text { if } t \leq T(1-2 \varepsilon) .\end{cases}
$$

We start from $\varepsilon=1$, and consider the derivative of $u^{\varepsilon}$ solution to (24) with respect to $\varepsilon: u_{\varepsilon}=\frac{d}{d \varepsilon} u^{\varepsilon}$ solves

$$
\partial_{t} u_{\varepsilon}+\frac{1}{2}\left(\sigma^{\varepsilon}\right)_{\varepsilon}^{2} \frac{\gamma^{\varepsilon}}{1-\lambda \gamma^{\varepsilon}}+\frac{1}{2}\left(\sigma^{\varepsilon}\right)^{2} \frac{s^{2} \partial_{s s} u_{\varepsilon}}{\left(1-\lambda \gamma^{\varepsilon}\right)^{2}}=0
$$


with $u^{\varepsilon}(T)=0$, where $\gamma^{\varepsilon}=s^{2} \partial_{s s} u^{\varepsilon}$ and $\left(\sigma^{\varepsilon}\right)_{\varepsilon}^{2}=\frac{d}{d \varepsilon}\left(\sigma^{\varepsilon}\right)^{2}$. If for some $\nu^{\prime}(\varepsilon)$ there holds

$$
v^{\prime} \leq 1-\lambda \gamma^{\varepsilon} \leq 1 / v^{\prime},
$$$$
\gamma^{\varepsilon} \text { is globally Lipschitz with respect to } s \text {, }
$$

$$
u^{\varepsilon} \in C_{s, t, \mathrm{loc}}^{3+\beta, \frac{3+\beta}{2}}
$$

then one can define $S^{\epsilon, t, s}$ the unique strong solution on $[t, T]$ to

$$
\begin{aligned}
d S_{t^{\prime}}^{\epsilon, t, s} & =S_{t^{\prime}}^{\epsilon, t, s} \frac{\sigma^{\varepsilon}}{1-\lambda \gamma^{\varepsilon}} d W_{t^{\prime}}^{\mathbb{P}}, \\
S_{t}^{\epsilon, t, s} & =s,
\end{aligned}
$$

and $u_{\varepsilon}$ can be found by the following representation formula:

$$
u_{\varepsilon}(t, s)=\mathbb{E}^{\mathbb{P}}\left(\int_{t}^{T} \frac{1}{2}\left(\sigma^{\varepsilon}\right)_{\varepsilon}^{2} \frac{\gamma^{\varepsilon}}{1-\lambda \gamma^{\varepsilon}}\left(t, S_{t^{\prime}}^{\epsilon, t, s}\right) d t^{\prime}\right),
$$

moreover by standard parabolic regularity, $u_{\varepsilon} \in C_{s, t, 1 \mathrm{loc}}^{3+\beta, \frac{3+\beta}{2}}$. Hence the linearized operator is invertible at a point $u^{\varepsilon}$ satisfying (68), (69), (70), and from the implicit functions theorem one can find a solution $u^{\varepsilon_{2}} \in C_{s, t, \text { loc }}^{3+\beta, \frac{3+\beta}{2}}$ for $\varepsilon_{2}$ close to $\varepsilon$.

Then (68), (69), (70) hold on $[0, T(1-\varepsilon)]$ as a consequence of Theorem 5.8, and on $[T(1-\varepsilon), T]$ thanks to Theorem 5.6.

Therefore, one can apply the continuity method (see [18]) to build the curve $u^{\varepsilon}, \varepsilon \in[1,0]$, and $u^{\varepsilon}$ enjoys, uniformly with respect to $\varepsilon$, the a priori estimates of Theorem 5.8.

Finally, $u^{0}$ solves (24), (25), (26).

\subsection{Existence and uniqueness results.}

THEOREM 5.15. 1. Let $\Phi, \sigma$ satisfy Assumptions 5.4, 5.5, there exists a classical solution to $(24),(25),(26)$.

2. If moreover $\Phi, \sigma$ satisfy the assumptions (i) to (iv) of Theorem 5.8, this solution is unique.

REMARKS. Those solutions will then naturally enjoy the a priori estimates of Theorems 5.8 or 5.12 , as they are satisfied for any classical solution.

ProOF OF THEOREM 5.15. One can approach $\Phi$ by a sequence $\Phi^{v}$ satisfying the assumptions (i) to (v) of Theorem 5.8. By letting $v$ go to 0 , the interior estimates do not depend on $v$, and using a standard compactness argument the sequence of solutions $u^{v}$ converges locally uniformly, and the a priori estimates pass to the limit. 
To prove the second point, we need the following result whose proof is deferred to Appendix C.

THEOREM 5.16. Let $u_{1}, u_{2} \in C_{s, t}^{3,1}\left([0, T] \times \mathbb{R}_{+}^{*}\right)$ satisfy, for some $C>0$,

$$
\begin{gathered}
-C \leq s^{2} \partial_{s s} u \leq \lambda^{-1}-1 / C, \\
\left|s^{2} \partial_{s s} u\right|+\left|s^{3} \partial_{s s s} u\right|+\left|s \partial_{s} \sigma\right| \leq C, \\
\partial_{t} u_{1}+\frac{\sigma^{2}(t, s)}{2} F\left(s^{2} \partial_{s s} u_{1}\right) \leq 0, \\
\partial_{t} u_{2}+\frac{\sigma^{2}(t, s)}{2} F\left(s^{2} \partial_{s s} u_{2}\right) \geq 0,
\end{gathered}
$$

with $F$ as in (13). Then if $u_{1}(T) \geq u_{2}(T), u_{1}(t) \geq u_{2}(t)$ on $[0, T]$.

We already know that the a priori estimate of Theorem 5.8 holds. Then applying Theorem 5.16 on $[0, T-\tau]$ (which is allowed using the interior a priori estimates), we obtain that

$$
t \rightarrow \sup _{s>0}\left\{\left|u_{1}(T-t, s)-u_{2}(T-t, s)\right|\right\}
$$

is nonincreasing. We can conclude the proof if we have for $u=u_{1}, u_{2}$ that

$$
\lim _{t \rightarrow T}\|u(t, \cdot)-\Phi(\cdot)\|_{L^{\infty}\left(\mathbb{R}_{+}^{*}\right)}=0 .
$$

This comes by considering the rescaled solution $u^{\alpha}$ introduced in the proof of Theorem 5.8. By a compactness argument, $u^{\alpha}(t, \cdot)$ converges to $u^{\alpha}(T, \cdot)$ in $C^{0}([-\lambda B-\theta,-\lambda B-1])$ as $t \rightarrow 0$, uniformly with respect to $\alpha$, which implies (71).

6. Representation of the solution. In what follows, we let $u$ be a classical solution to (24), (25), (26) as in Definition 5.1. We consider as above $W_{t}^{\mathbb{P}}$ a standard Brownian motion on a filtered probability space $\left(\Omega, \mathbb{F}, \mathcal{F}_{t}, \mathbb{P}\right)$. Since $u$ is only locally smooth up to time $T$, we first need to introduce a notion of local solution to (34), (35), (36), defined up to a stopping time; see [19], Chapter 1.

Definition 6.1. For $(t, s) \in[0, T) \times \mathbb{R}_{+}^{*}$, a pair $\left(S^{t, s}, \tau^{t, s}\right)$ is called a local strong solution to (34), (35), (36) if $\tau^{t, s}$ is a stopping time of the filtration $\left\{\mathcal{F}_{t}\right\}$, with $\tau^{t, s} \in(t, T]$, such that

$$
\exists M(t, s) \in \mathbb{R} \quad \text { such that }
$$

$$
\sup \left\{\frac{\sigma\left(r, S_{r}^{t, s}\right)}{1-\lambda \gamma\left(r, S_{r}\right)}+\left|\gamma\left(r, S_{r}\right)\right|, r \in\left[t, \tau^{t, s}\right]\right\}=M(t, s) \quad \mathbb{P} \text {-a.s. }
$$


and $\left(S_{r}^{t, s}\right)_{r \in[t, T]}$ is a strong solution on $[t, T]$ to

$$
\frac{d S_{r}}{S_{r}}=\mathbb{I}_{r \leq \tau} \frac{\sigma\left(r, S_{r}\right)}{1-\lambda \gamma\left(r, S_{r}\right)} d W_{r}^{\mathbb{P}},
$$

with the initial condition $S_{t}^{t, s}=s$, and where $\gamma=s^{2} \partial_{s s} u$.

Under the additional assumption that $u \in C_{s, t, \text { loc }}^{3,1}$, we have the following existence result.

Proposition 6.2. 1. Under the above assumptions, there exists a local strong solution to (34), (35), (36) for all $t \in[0, T], s>0$.

2. For all $t \in[0, T], s>0$, and for any $\left(S^{1}, \tau_{1}\right),\left(S^{2}, \tau_{2}\right)$ local strong solutions to (34), (35), (36) (with the same initial condition $S_{t}=s$ ), there holds

$$
S_{r \wedge \tau_{2}}^{1}=S_{r \wedge \tau_{1}}^{2} \quad \mathbb{P} \text {-a.s. }
$$

3. If $F(\gamma(t, s))$ [defined in (25)] is globally bounded, then there exists a unique global strong solution to (34), (35), (36).

4. The solution to (73) is a true martingale up to time $T$.

ProOf. Let $t<T, s>0, v>0$ be fixed. Under our assumptions, $u$ has enough regularity to define a strong solution $S^{t, s}$ to (34), (35), (36) up to the stopping time:

$$
\tau_{v}=\inf \left\{r \in[t, T], F\left(\gamma\left(r, S_{r}^{t, s}\right)\right) \notin\left[-\frac{1}{\lambda}+v, v^{-1}\right]\right\} \wedge T-v .
$$

For $v$ small enough, there will hold $F(\gamma(t, s)) \in\left(-\frac{1}{\lambda}+v, v^{-1}\right)$ and, therefore, $\tau_{v}>t \mathbb{P}$-a.s. Hence there exists $v$ small enough so that $\left(S^{t, s}, \tau_{v}\right)$ is a local strong solution.

The point (2) follows directly from the definition (73) and from the Lipschitz regularity of the coefficients.

If $F$ is globally bounded, being already locally Lipschitz, one can construct a unique global strong solution to (34) (see [17], Theorem 2.2, page 104).

To see why a local strong solution is a true martingale, we use the property (72), which implies that Novikov's condition (see [28]) is satisfied, and hence the martingale property.

Following the notation introduced previously, we recall

$$
\begin{aligned}
V(t, s) & =\ln (s)+\lambda\left(u(t, s)-s \partial_{s} u(t, s)\right), \\
\mathcal{S}(t, \cdot) & =[V(t, \cdot)]^{-1} .
\end{aligned}
$$


6.1. A maximum principle for the second derivative. We state this result of independent interest for $F$ as in (25), but it is valid for a large range of nonlinear diffusions. The result will be used in the proof of Theorem 6.6.

THeOREM 6.3. Let $\sigma \in C_{t, s}^{1,1}\left([0, T] \times \mathbb{R}_{+}^{*}\right)$ satisfy Assumption 5.4, with $\sup _{s>0, t \geq 0}\left\{\left|\partial_{t} \sigma(t, s)\right|\right\}<+\infty$ and $\partial_{t} \sigma \in C_{s, t, \mathrm{loc}}^{\alpha, \alpha^{\prime}}\left([0, T] \times \mathbb{R}_{+}^{*}\right)$ for some $\alpha, \alpha^{\prime}>0$. Then:

(i) Any classical solution to (24), (25), (26) belongs to $C_{s, t, l o c}^{3,1}\left([0, T) \times \mathbb{R}_{+}^{*}\right)$.

(ii) Let $\left(S^{t, s}, \tau^{t, s}\right)$ be a local strong solution of (34), (35), (36). There holds

$$
\left(\sigma^{2} F\left(s^{2} \partial_{s s} u\right)\right)(t, s)=\mathbb{E}^{\mathbb{P}}\left\{\left(\sigma^{2} F\left(s^{2} \partial_{s s} u\right)\right)\left(\tau^{t, s}, S_{\tau^{t, s}}^{t, s}\right) e^{-\int_{t}^{\tau^{t, s}} \frac{\partial_{t}\left(\sigma^{2}\right)}{\sigma^{2}}\left(t^{\prime}, S_{t^{\prime}}^{t, s}\right)} d t^{\prime}\right\} .
$$

(iii) If there exists a sequence of local strong solutions $\left(S^{t, s, n}, \tau_{n}^{t, s}\right)_{n \in \mathbb{N}}$ of (34), (35), (36) such that

$$
\begin{aligned}
\lim _{n \rightarrow+\infty} \tau_{n}^{t, s} & =T \quad \mathbb{P} \text {-a.s., } \\
\exists S_{T}^{t, s} & =\lim _{n \rightarrow+\infty} S_{\tau_{n}^{t, s}}^{t, s, n} \in \mathbb{R}_{+}^{*} \quad \mathbb{P} \text {-a.s. }
\end{aligned}
$$

then

$$
\left(\sigma^{2} F\left(s^{2} \partial_{s s} u\right)\right)(t, s) \geq \mathbb{E}^{\mathbb{P}}\left\{\left(\sigma^{2} F\left(s^{2} \partial_{s s} u\right)\right)\left(T, S_{T}^{t, s}\right) e^{-\int_{t}^{T} \frac{\partial_{t}\left(\sigma^{2}\right)}{\sigma^{2}}\left(t^{\prime}, S_{t^{\prime}}^{t, s}\right)} d t^{\prime}\right\} .
$$

(iv) Assuming that $F(\gamma(t, s))$ is globally bounded, there exists a unique global strong solution $S^{t, s}$ of (34), (35), (36) and

$$
\left(\sigma^{2} F\left(s^{2} \partial_{s s} u\right)\right)(t, s)=\mathbb{E}^{\mathbb{P}}\left\{\left(\sigma^{2} F\left(s^{2} \partial_{s s} u\right)\right)\left(T, S_{T}^{t, s}\right) e^{-\int_{t}^{T} \frac{\partial_{t}\left(\sigma^{2}\right)}{\sigma^{2}}\left(t^{\prime}, S_{t^{\prime}}^{t, s}\right)} d t^{\prime}\right\} .
$$

PROOF. We proceed by localisation and approximation. By our previous results, we already know that $u \in C_{s, t, \text { loc }}^{2+\alpha, 1+\alpha / 2}\left([0, T) \times \mathbb{R}_{+}^{*}\right)$ for any $\alpha \in[0,1)$. We approximate $\sigma$ by $\sigma^{\varepsilon} \in C^{\infty}\left([0, T] \times \mathbb{R}_{+}^{*}\right)$, converging locally to $\sigma$ as $\varepsilon \rightarrow 0$. By standard parabolic regularity, on $Q^{v}=[0, T-v) \times(v, 1 / v)$ the solution $u^{v, \varepsilon}$ to (24), with $\sigma^{\varepsilon}$ instead of $\sigma$, and with $u^{v, \varepsilon}=u$ on the parabolic boundary of $Q^{v}$ is $C^{\infty}$ smooth in the interior of $Q^{v}$ and $C_{t, s}^{2+\alpha, 1+\alpha / 2}$ globally in $Q^{v}$ (see [25]). Then $u^{\nu, \varepsilon}$ is smooth enough to differentiate the equation, and

$$
\Theta^{\nu, \varepsilon}=-\partial_{t} u^{\nu, \varepsilon}=\frac{1}{2}\left(\sigma^{\varepsilon}\right)^{2}(t, s) F\left(\gamma^{\nu, \varepsilon}\right)
$$

solves

$$
\partial_{t} \Theta^{v, \varepsilon}+\frac{\left(\sigma^{\varepsilon}\right)^{2} s^{2}}{2} F^{\prime}\left(\gamma^{v, \varepsilon}\right) \partial_{s s} \Theta^{\nu, \varepsilon}=\frac{\partial_{t}\left(\left(\sigma^{\varepsilon}\right)^{2}\right)}{\left(\sigma^{\varepsilon}\right)^{2}} \Theta^{v, \varepsilon},
$$

in $Q^{v}$, with $\Theta^{v, \varepsilon}=\partial_{t} u$ on the parabolic boundary of $Q^{v}$. Under the present assumptions, the coefficients of this equation are uniformly (with respect to $\varepsilon$ ) Hölder 
continuous, and by Schauder regularity this implies $\Theta^{\nu, \varepsilon} \in C_{s, t, \text { loc }}^{2+\alpha, 1+\alpha / 2}$, uniformly with respect to $\varepsilon$. Since $\sigma \in C_{s, t}^{1,1}$, this implies that $F\left(\gamma^{\nu, \varepsilon}\right)$, and hence $\gamma^{\nu, \varepsilon}$ are (locally in $t, s$, uniformly with respect to $\varepsilon$ ) Lipschitz with respect to $s, t$.

It follows that the sequence $u^{v, \varepsilon}$ converges locally to $\tilde{u}$ solution to (24). As we are on a bounded domain, the solution to (24) in $Q^{v}$ is unique, once the boundary condition is prescribed, therefore, $\tilde{u}=u$. Then $\Theta^{\nu, \varepsilon}$ converges to $\Theta$ in $Q^{v}$, and we have proved point (i).

To prove point (ii), we consider a local strong solution $\left(S^{t, s}, \tau^{t, s}\right)$. Then $\Theta$ solves (78) in $Q^{v}$ for $\varepsilon=0$. Letting $\theta_{\nu}$ be the first exit time of $S^{t, s}$ out of $Q_{\nu}$, we have

$$
\Theta(t, s)=\mathbb{E}^{\mathbb{P}}\left\{\Theta\left(\tau^{t, s} \wedge \theta_{\nu}, S_{\tau^{t, s} \wedge \theta_{\nu}}^{t, s}\right) e^{-\int_{t}^{t, s} \wedge \theta_{\nu} \frac{\partial_{t}\left(\sigma^{2}\right)}{\sigma^{2}}}\right\} .
$$

We claim that

LEMma 6.4. If $\left(S^{t, s}, \tau^{t, s}\right)$ is a local strong solution, there holds $\theta_{v} \wedge \tau^{t, s} \rightarrow$ $\tau^{t, s}$ as $v$ goes to $0, \mathbb{P}$-a.s.

PROOF. From property (72), the diffusion and the drift of the process $\ln \left(S^{t, s}\right)$ are bounded up to $\tau^{t, s}$, therefore, $\mathbb{E}\left(\mid \ln \left(\left.S_{\tau^{t, s}}^{t, s}\right|^{2}\right)<+\infty\right.$. By Doob's maximal inequality, it follows that $\mathbb{E}\left(\sup \left\{\left|\ln \left(S_{r}^{\nu, t, s}\right)\right|, r \in\left[t, \tau^{t, s}\right]\right\}\right)<+\infty$, and the result follows.

To conclude the proof of point (ii), we use the property (72), which, by dominated convergence, implies that one can pass to the limit in (79), so the equality (75) remains.

To prove point (iv), we first observe that under the assumptions and point (i), for each $(t, s) \in Q^{v}$, there exists a unique global strong solution of to (34), (35), (36) (cf. Proposition 6.2), and it is also a local strong solution with $\tau^{t, s} \equiv T$. We apply directly point (ii) to conclude.

To prove point (iii), we use point (ii) and Fatou's lemma since $F$ is bounded by below.

We now introduce a modified process $Y_{t}$ that will be key to study the properties of the process $S_{t}$.

Proposition 6.5. Let $\Phi, \sigma$ satisfy the assumptions of Theorem 5.12. Let $u$ be a $C_{s, t, 10 c}^{3,1}$ classical solution to $(24),(25),(26)$, and $\left(S_{t}, \tau\right)$ be a local strong solution to (34). Let $V(t, s)$ be defined from $u$ as above. Consider the process

$$
\begin{aligned}
Y_{t} & =V\left(t, S_{t}\right) \\
& =\ln \left(S_{t}\right)+\lambda\left(u\left(t, S_{t}\right)-S_{t} \partial_{s} u\left(t, S_{t}\right)\right) .
\end{aligned}
$$


Then $Y$ satisfies up to time $\tau$

$$
d Y_{t}=\sigma\left(\left(d W_{t}^{\mathbb{P}}-s \partial_{s} \sigma d t\right)+\frac{s \partial_{s} \sigma-\sigma}{1-\lambda \gamma} d t\right)+\frac{\sigma^{2}}{2} d t
$$

REMARK. When $\lambda=0$, we recover that $d \ln \left(S_{t}\right)=\sigma d W_{t}^{\mathbb{P}}-\sigma^{2} / 2 d t$.

ProOF OF PROPOSITION 6.5. We have that

$$
\begin{aligned}
d u\left(t, S_{t}\right) & =\partial_{s} u\left(t, S_{t}\right) d S_{t}+\frac{\lambda \gamma^{2} \sigma^{2}}{2(1-\lambda \gamma)^{2}} d t \\
d\left(S_{t} \partial_{s} u\left(t, S_{t}\right)\right) & =\left(\partial_{s} u+S_{t} \partial_{s s} u\right) d S_{t}+\left(\partial_{s s} u+\frac{1}{2} S_{t} \partial_{s s s} u\right) \frac{\sigma^{2} S_{t}^{2}}{(1-\lambda \gamma)^{2}}+S_{t} \partial_{t s} u \\
d \ln \left(S_{t}\right) & =\frac{d S_{t}}{S_{t}}-\frac{1}{2} \frac{\sigma^{2}}{(1-\lambda \gamma)^{2}}, \\
-\partial_{t s} u & =\frac{1}{2} \partial_{s}\left(\sigma^{2}\right) \frac{\gamma}{1-\lambda \gamma}+\frac{\sigma^{2}}{2} \frac{2 S_{t} \partial_{s s} u+S_{t}^{2} \partial_{s s s} u}{(1-\lambda \gamma)^{2}}
\end{aligned}
$$

Rearranging the terms, we obtain that

$$
\begin{aligned}
\frac{1}{\lambda} d Y_{t} & =\left(\frac{1}{\lambda}-\gamma\right) \frac{d S}{S}-\frac{\sigma^{2}}{\lambda(1-\lambda \gamma)} d t+\frac{1}{2} \frac{\gamma s \partial_{s}\left(\sigma^{2}\right)}{1-\lambda \gamma} d t \\
& =\frac{\sigma}{\lambda} d W_{t}^{\mathbb{P}}-\frac{\sigma^{2}}{\lambda(1-\lambda \gamma)} d t+\frac{\sigma s \partial_{s} \sigma}{\lambda}\left(-1+\frac{1}{1-\lambda \gamma}\right)+\frac{\sigma^{2}}{2 \lambda} d t,
\end{aligned}
$$

and the result follows.

6.2. Dual representation formula and martingale property under $\mathbb{P}$. Based on the variational formulation (3), we now have a representation result for the solution $u$, as well as a martingale property for $S_{t}$ solution of (34), (35), (36).

THEOREM 6.6. Let $\Phi$ satisfy Assumption 5.5, and assume moreover that

$$
\mathcal{S}_{T}(y) \text { is finite for every } y \in \mathbb{R},
$$

with $\mathcal{S}_{T}$ as in (55). Let $\sigma$ satisfy the assumptions of Theorem 6.3, and assume moreover that $\left(s+s^{2}\right)\left|\partial_{s} \sigma\right|$ is bounded. Let $u$ be a classical solution to (24), (25), (26). Then:

- For all $(t, s) \in[0, T] \times \mathbb{R}_{+}^{*}$, there exists a unique global strong solution $S^{t, s}$ to (34), (35), (36) on $[t, T]$,

- $S^{t, s}$ is a martingale on $[0, T]$, 
- (40) holds, that is,

$$
u(t, s)=\mathbb{E}^{\mathbb{P}}\left(\Phi\left(S_{T}^{t, s}\right)-\frac{1}{2 \lambda} \int_{0}^{T}\left(\frac{\sigma\left(r, S_{r}^{t, s}\right)}{1-\lambda \gamma\left(r, S_{r}^{t, s}\right)}-\sigma\left(r, S_{r}^{t, s}\right)\right)^{2} d r\right),
$$

[although $\mathbb{E}^{\mathbb{P}}\left(\Phi\left(S_{T}^{t, s}\right)\right)$ may not be finite, and then (83) should be understood in the sense of (91)], and moreover

$$
u(t, s)=\sup _{a \in \mathcal{A}_{T}} \mathbb{E}^{\mathbb{P}}\left(\Phi\left(S_{T}^{a, t, s}\right)-\frac{1}{2 \lambda} \int_{0}^{T}\left(\left(a_{r}\right)^{1 / 2}-\sigma\left(t, S_{r}^{a, t, s}\right)\right)^{2} d r\right),
$$

where $a, \mathcal{A}_{T}$ and $S^{a, t, s}$ are defined in (43), (44), (45),

$$
\begin{aligned}
& \mathbb{P}\left\{\lambda\left(S_{T}^{t, s}\right)^{2} \partial_{s s} \Phi\left(S_{T}^{t, s}\right)=1\right\}=0, \\
& \mathbb{E}^{\mathbb{P}}\left(\frac{1}{1-\lambda\left(S_{T}^{t, s}\right)^{2} \partial_{s s} \Phi\left(S_{T}^{t, s}\right)}\right)<+\infty,
\end{aligned}
$$

- identity (40) holds for any $\Psi \leq \Phi$ such that $(\Psi-\Phi)\left(1-\lambda s^{2} \partial_{s s} \Phi\right)=0$,

- if $\Phi(s) \leq C(1+s)+\left(\varepsilon_{0}-\lambda\right) \ln (s)$ for some $C, \varepsilon_{0}>0$, then

$$
\mathbb{E}^{\mathbb{P}}\left(\int_{0}^{T} \frac{\sigma^{2}\left(r, S_{r}^{t, s}\right)}{\left(1-\lambda \gamma\left(r, S_{r}^{t, s}\right)\right)^{2}} d t\right)<+\infty
$$

and $\mathbb{E}^{\mathbb{P}}\left(\Phi\left(S_{T}^{t, s}\right)\right)$ is finite.

REMARK. As we will see, condition (82) implies $\lim _{s \rightarrow+\infty} V_{T}(s)=+\infty$, and thus implies to be in the second case of Point 4 in Proposition 5.3.

PROOF. From now on, unless specified otherwise, we consider the solution to (34), (35), (36) starting from $s=S_{0}$ at time $t=0$, and we will drop the superscript $\left(0, S_{0}\right)$ for simplicity. Our result will remain true when changing $\left(0, S_{0}\right)$ to any $(t, s)$ with $t \geq 0, s>0$. Consider the stopping time $\tau_{v}$ as in (74) and $S_{t}^{v}$, the process stopped at time $\tau_{\nu}$. The pair $\left(S_{t}^{\nu}, \tau_{v}\right)$ is a local strong solution to (34), (35), (36) (see Definition 6.1). We then have the following proposition.

PROPOSITION 6.7. The process $S_{t}^{\nu}$ defined above is a martingale on $[0, T]$, and under the probability $\mathbb{Q}^{v}$ given by

$$
\left.\frac{d \mathbb{Q}^{v}}{d \mathbb{P}}\right|_{\mathcal{F}_{t}}=\frac{S_{t}^{v}}{S_{0}}
$$

$Y_{t}$, defined in (80), satisfies on $\left[0, \tau_{v}\right]$

$$
d Y_{t}=\sigma d W^{\mathbb{Q}^{\nu}}+\left(s \sigma \partial_{s} \sigma\left(-1+\frac{1}{1-\lambda \gamma}\right)+\frac{\sigma^{2}}{2}\right) d t
$$

for $W^{\mathbb{Q}^{v}}$ a Brownian motion under $\mathbb{Q}^{v}$. If moreover $S$ is a martingale up to time $T$, then we define $\mathbb{Q}$ accordingly and (88) holds up to time $T$. 
Proof. From Proposition 6.2, $S_{t}^{v}$ it is a true martingale. Using (81), the rest of proof is a straightforward application of Girsanov's theorem (see [22]).

To prove (85), we use the representation formula (75). Given the uniform bounds we have on $u$ and $F(\gamma)$ from Theorem 5.12, we claim that there exists a constant $C$ independent of $v$ such that, for $M>0$ large,

$$
\mathbb{P}\left\{\sup _{t \in\left[0, \tau_{\nu}\right]}\left\{F\left(\gamma\left(t, S_{t}^{\nu}\right)\right)\right\} \geq M\right\} \leq \frac{C}{M} .
$$

Indeed, if this is not true, by formula (75) one can show that $F\left(\gamma\left(0, S_{0}\right)\right) \geq C$ for any $C>0$, which contradicts the regularity result of Theorem 5.12.

Letting

$$
\Omega_{v}^{M}=\left\{\sup _{t \in\left[0, \tau_{\nu}\right]}\left\{F\left(\gamma\left(t, S_{t}^{\nu}\right)\right)\right\} \leq M\right\}
$$

we see that the sequence $\Omega_{v}^{M}$ is decreasing when $v \rightarrow 0$, therefore, has a limit $\Omega^{M}$ for which $\mathbb{P}\left(\Omega^{M}\right) \geq 1-C / M$. In $\Omega^{M}$, the diffusion and the drift of $\ln \left(S_{t}^{v}\right)$ are bounded $\mathbb{P}$-a.s by a function of $M$. Therefore,

$$
\mathbb{P}\left(\Omega^{M} \cap\left\{\sup _{t \in\left[0, \tau_{\nu}\right]}\left|\ln \left(S_{t}^{\nu}\right)\right| \geq N\right\}\right) \leq \varepsilon(N),
$$

with $\lim _{N \rightarrow \infty} \varepsilon(N)=0$. We claim that this implies

$$
\mathbb{P}\left(\Omega^{M} \cap\left\{\lim _{\nu \rightarrow 0} \tau_{\nu} \leq T-1 / N\right\}\right)=0 .
$$

Indeed, since $F(\gamma) \in C_{\text {loc }}^{0}\left([0, T) \times \mathbb{R}_{+}^{*}\right)$, the set $\left\{\lim _{v \rightarrow 0} \tau_{v} \leq T-1 / N\right\}$ corresponds to trajectories such that $\ln \left(S_{t}^{\nu}\right)$ leaves any compact set before $T-1 / N$. We conclude using (90).

We have then

$$
\mathbb{P}\left(\left\{\lim _{\nu \rightarrow 0} \tau_{\nu}=T\right\}\right) \geq \mathbb{P}\left(\Omega^{M} \cap\left\{\lim _{\nu \rightarrow 0} \tau_{\nu}=T\right\}\right)=\mathbb{P}\left(\Omega^{M}\right),
$$

and letting $M \rightarrow 0$, we conclude that $\tau_{v} \rightarrow T \mathbb{P}$-a.s. We therefore can define $S$ up to $T^{-}$and from (89), $\sup _{t \in[0, T)}\left\{F\left(\gamma\left(t, S_{t}\right)\right\}\right.$ is finite $\mathbb{P}$-a.s., which shows that, $\mathbb{P}$-almost surely, $S_{t}$ is continuous up to time $T$ and $S_{T}^{2} \partial_{S S} \Phi\left(S_{T}\right)<1$.

We now prove that $S_{t}$ is a martingale up to time $T$. The sequence $S_{t}^{v}$ is a sequence of martingales up to time $T$ that satisfies $\mathbb{E}^{\mathbb{P}}\left(S_{t}^{\nu}\right)=S_{0}$ for all $v>0$, $t \in[0, T]$. If we can show that the family $S_{t}^{\nu}$ is uniformly integrable, and that it converges to $S_{t}$, then $S_{t}$ satisfies $\mathbb{E}^{\mathbb{P}}\left(S_{t}\right)=S_{0}$, and by standard arguments, as $S_{t}$ is nonnegative, this implies that $S_{t}$ is a martingale up to time $T$. We consider the family $V^{v}(t, s)=V\left(t \wedge \tau_{\nu}, s\right)$, and its inverse $\mathcal{S}^{v}(t, y)=\mathcal{S}\left(t \wedge \tau_{v}, y\right)$, and we have using Proposition 6.7,

$$
\begin{aligned}
\mathbb{E}^{\mathbb{P}}\left(S_{t}^{v} \mathbb{I}_{S_{t}^{v} \geq M}\right) & =S_{0} \mathbb{E}^{\mathbb{Q}^{v}}\left(\mathbb{I}_{S_{t}^{v} \geq M}\right) \\
& =S_{0} \mathbb{Q}^{v}\left\{\mathcal{S}^{v}\left(t, Y_{0}+Z_{t}^{v}+A_{t}^{v}\right) \geq M\right\} \\
& =S_{0} \mathbb{Q}^{v}\left\{Y_{0}+Z_{t}^{v}+A_{t}^{v} \geq V^{v}(t, M)\right\}
\end{aligned}
$$


where

$$
\begin{aligned}
& Z_{t}^{v}=\int_{0}^{t \wedge \tau_{v}} \sigma\left(t, S_{t}^{v}\right) d W_{t}^{\mathbb{Q}^{v}} \\
& A_{t}^{v}=\int_{0}^{t \wedge \tau_{v}}\left(s \sigma \partial_{s} \sigma\left(-1+\frac{1}{1-\lambda \gamma}\right)+\frac{\sigma^{2}}{2}\right) d t .
\end{aligned}
$$

Letting

$$
V^{\infty}(s)=\inf \{V(t, s), t \in[0, T]\},
$$

we first we observe that by Chebyshev's inequality

$$
\mathbb{E}^{\mathbb{P}}\left(S_{t}^{\nu} \mathbb{I}_{S_{t}^{\nu} \geq M}\right) \leq S_{0}\left(V^{\infty}(M)\right)^{-1} \mathbb{E}^{\mathbb{Q}^{v}}\left(Y_{0}+\left|Z_{t}^{v}\right|+\left|A_{t}^{v}\right|\right)
$$

Then we have the following.

LeMma 6.8. Under Assumptions (82) and 5.5, there holds $\lim _{s \rightarrow \infty} V^{\infty}=$ $+\infty$.

PROOF. Indeed remember that $\mathcal{S}$ is the inverse of $V$ and that $1 / \mathcal{S}=\partial_{x} w$. This is then an consequence of Lemma A.1 combined with the concavity of $w$ : under Assumption (82), $\partial_{x} w$ is bounded away from 0 on every set $[0, T] \times A, A$ bounded.

Finally, $Z^{v}$ is bounded in $L^{1}\left(\mathbb{Q}^{v}\right)$ uniformly with respect to $t, v$ and if we have a uniform $L^{1}$ bound on $A^{v}$, using Lemma 6.8 , we have shown that $\mathbb{E}^{\mathbb{P}}\left(S_{t}^{v} \mathbb{I}_{S^{\nu} \geq M}\right)$ converges to 0 as $M \rightarrow \infty$, uniformly with respect to $t \in[0, T], v>0$. This shows the uniform integrability of the family. Having observed above that $S_{t}$ is almost surely continuous up to time $T, S_{T}^{\nu}$ converges a.s. to $S_{T}$, hence $E^{\mathbb{P}}\left(S_{T}\right)=S_{0}$ which allows to conclude. It thus remains to show the following.

LEMMA 6.9. Under the assumptions of Theorem 6.6, $A_{t}^{v}$ is bounded in $L^{1}\left(\mathbb{Q}^{v}\right)$, uniformly with respect to $t, v$.

PROOF. We will use Theorem 6.3 and (75). We need to bound

$$
\begin{aligned}
& \mathbb{E}^{\mathbb{Q}^{v}}\left(\int_{0}^{t \wedge \tau_{v}} S_{t^{\prime}} \sigma\left|\partial_{s} \sigma\right| \frac{1}{1-\lambda \gamma} d t^{\prime}\right) \\
& \quad=S_{0}^{-1} \mathbb{E}^{\mathbb{P}}\left(\int_{0}^{t \wedge \tau_{v}} S_{t^{\prime}}^{2} \sigma\left|\partial_{s} \sigma\right| \frac{1}{1-\lambda \gamma} d t^{\prime}\right),
\end{aligned}
$$

which, assuming a bound on $s^{2} \partial_{s} \sigma$ is equivalent to a bound on

$$
\mathbb{E}^{\mathbb{P}}\left(\int_{0}^{t \wedge \tau_{v}} F\left(\gamma\left(t^{\prime}, S_{t^{\prime}}\right)\right) d t\right)
$$


and which follows directly from the fact that

$$
\begin{aligned}
& \mathbb{E}^{\mathbb{P}}\left(\int_{0}^{t \wedge \tau_{v}} F\left(\gamma\left(t^{\prime}, S_{t^{\prime}}\right) d t^{\prime}\right)\right. \\
& \quad=\int_{0}^{t} \mathbb{E}^{\mathbb{P}}\left(F\left(\gamma\left(t^{\prime} \wedge \tau_{\nu}, S_{t^{\prime} \wedge \tau_{v}}\right)\right)-F\left(\gamma\left(\tau_{v}, S_{\tau_{v}}\right)\right) \mathbb{I}_{t^{\prime} \geq \tau_{v}}\right) d t^{\prime} \\
& \quad \leq C_{1}(\sigma) T F\left(\gamma\left(0, S_{0}\right)\right)+C_{2}(\sigma, \lambda, T),
\end{aligned}
$$

from Theorem 6.3. Since $F\left(\gamma\left(0, S_{0}\right)\right)$ is bounded by Theorem 5.12, the result follows.

To prove (87), we use again the stopped process $S_{t}^{v}$. Then for $v>0$, (40) holds up to $\tau_{v}$, that is,

$$
u\left(0, S_{0}\right)=\mathbb{E}^{\mathbb{P}}\left(u\left(\tau_{v}, S_{\tau_{v}}\right)-\frac{1}{2 \lambda} \int_{0}^{\tau_{v}}\left(\sigma^{\gamma}-\sigma\right)^{2} d t\right),
$$

where $\sigma^{\gamma}(t, s)$ is as in (35). Under our assumptions, by the result of Proposition 5.9, $u$ satisfies for some $C>0, u(t, s) \leq C(1+s)+\left(\varepsilon_{0}-\frac{1}{\lambda}\right) \ln (s)$. This combined with the above identity implies that

$$
\begin{aligned}
u\left(0, S_{0}\right) \leq & C\left(1+S_{0}\right)-\frac{1}{2} \varepsilon_{0} \mathbb{E}^{\mathbb{P}}\left(\int_{0}^{\tau_{v}}\left(\sigma^{\gamma}\right)^{2} d t\right) \\
& +\frac{1}{2 \lambda} \mathbb{E}^{\mathbb{P}}\left(\int_{0}^{\tau_{v}}\left(\sigma^{\gamma}\right)^{2}-\left(\sigma^{\gamma}-\sigma\right)^{2} d t\right),
\end{aligned}
$$

hence, since $u\left(0, S_{0}\right)$ is finite, and using Cauchy-Schwarz's inequality, $\mathbb{E}^{\mathbb{P}}\left(\int_{0}^{\tau_{v}}\left(\sigma^{\gamma}\right)^{2} d t\right)$ is uniformly bounded as $v$ goes to 0 .

We now prove that identity (40) holds even when $u$ is not smooth up to time $T$. We consider the stopping times $\tau_{v}$. Then there will hold by Itô's formula

$$
\begin{aligned}
u\left(0, S_{0}\right) & =\mathbb{E}^{\mathbb{P}}\left(u\left(\tau_{v}, S_{\tau_{v}}\right)-\frac{1}{2 \lambda} \int_{0}^{\tau_{v}}\left(\sigma^{\gamma}-\sigma\right)^{2} d t\right) \\
& =\mathbb{E}^{\mathbb{P}}\left(v\left(\tau_{v}, S_{\tau_{v}}\right)-\frac{1}{\lambda} \ln \left(S_{\tau_{v}}\right)-\frac{1}{2 \lambda} \int_{0}^{\tau_{v}}\left(\sigma^{\gamma}-\sigma\right)^{2} d t\right) \\
& =\mathbb{E}^{\mathbb{P}}\left(v\left(\tau_{v}, S_{\tau_{v}}\right)+\frac{1}{\lambda} \int_{0}^{\tau_{v}} \sigma^{\gamma} \sigma-\frac{1}{2} \sigma^{2} d t\right),
\end{aligned}
$$

where $v(t, s)=\frac{1}{\lambda} \ln (s)+u$. By monotone convergence, $\int_{0}^{\tau_{v}} \sigma^{\gamma} \sigma d t$ converges to $\int_{0}^{T} \sigma^{\gamma} \sigma d t$, and arguing as Lemma $6.9, \mathbb{E}^{\mathbb{P}}\left(\int_{0}^{T} \sigma^{\gamma}\left(t, S_{t}\right) d t\right)$ is bounded under the assumption that $\left(s+s^{2}\right) \partial_{s} \sigma$ is bounded. Then under (31), $v$ is bounded above by $C(1+s)$, hence by Fatou's lemma,

$$
\begin{aligned}
\limsup \mathbb{E}^{\mathbb{P}}\left(v\left(\tau_{v}, S_{\tau_{v}}\right)-C\left(1+S_{\tau_{v}}\right)\right) & \leq \mathbb{E}^{\mathbb{P}}\left(v\left(T, S_{T}\right)-C\left(1+S_{T}\right)\right) \\
& =\mathbb{E}^{\mathbb{P}}\left(v\left(T, S_{T}\right)-C\left(1+S_{0}\right)\right)
\end{aligned}
$$


as we know already that $S$ is a martingale. We then have the first inequality

$$
u\left(0, S_{0}\right) \leq \mathbb{E}^{\mathbb{P}}\left(v\left(T, S_{T}\right)+\frac{1}{\lambda} \int_{0}^{T} \sigma^{\gamma} \sigma-\frac{1}{2} \sigma^{2} d t\right) .
$$

On the other hand, we know that $v$ is concave; hence (and using again that $S$ is a martingale)

$$
\begin{aligned}
\mathbb{E}^{\mathbb{P}}\left(v\left(\tau_{v}, S_{\tau_{v}}\right)\right) \geq & \mathbb{E}^{\mathbb{P}}\left(v\left(\tau_{\nu}, S_{T}\right)-\partial_{s} v\left(\tau_{\nu}, S_{\tau_{v}}\right)\left(S_{T}-S_{\tau_{v}}\right)\right) \\
= & \mathbb{E}^{\mathbb{P}}\left(v\left(T, S_{T}\right)\right)+\mathbb{E}^{\mathbb{P}}\left(v\left(\tau_{\nu}, S_{T}\right)-v\left(T, S_{T}\right)\right) \\
= & \mathbb{E}^{\mathbb{P}}\left(v\left(T, S_{T}\right)\right)+\mathbb{E}^{\mathbb{P}}\left(v\left(\tau_{v}, S_{T}\right)-v\left(T, S_{T}\right)-C\left(\tau_{v}-T\right)\right) \\
& +C \mathbb{E}^{\mathbb{P}}\left(\tau_{v}-T\right),
\end{aligned}
$$

where $C$ is chosen so that $v-C t$ is nonincreasing (such a $C$ exists since $F$ is bounded by below). By monotone convergence, the second term goes to 0 and the third converges easily to 0 , which shows that

$$
u\left(0, S_{0}\right) \geq \mathbb{E}^{\mathbb{P}}\left(v\left(T, S_{T}\right)+\frac{1}{\lambda} \int_{0}^{T} \sigma^{\gamma} \sigma-\frac{1}{2} \sigma^{2} d t\right),
$$

and equality thus follows:

$$
u\left(0, S_{0}\right)=\mathbb{E}^{\mathbb{P}}\left(\left(\Phi+\frac{1}{\lambda} \ln \right)\left(S_{T}\right)+\frac{1}{\lambda} \int_{0}^{T} \sigma^{\gamma} \sigma-\frac{1}{2} \sigma^{2} d t\right) .
$$

REMARK. This equality holds true even if $\mathbb{E}^{\mathbb{P}}(\Phi)=+\infty$, as

$$
\mathbb{E}^{\mathbb{P}}\left(\left(\Phi+\frac{1}{\lambda} \ln \right)\left(S_{T}\right)\right)
$$

will always be finite under the assumptions of the theorem. When $\mathbb{E}^{\mathbb{P}}(\Phi)$ is finite, (91) is equivalent to (83).

Following the same lines, for any element $a$ of $\mathcal{A}_{T}$ one can reproduce the computations of Section 4 and find that

$$
\begin{aligned}
\mathbb{E}^{\mathbb{P}}\left(u\left(\tau_{\nu}, S_{\tau_{v}}\right)-\frac{1}{2 \lambda} \int_{0}^{\tau_{v}}\left(\sigma^{\gamma}-\sigma\right)^{2} d t\right) \\
\geq \mathbb{E}^{\mathbb{P}}\left(u\left(\tau_{\nu}, S_{\tau_{v}}^{a}\right)-\frac{1}{2 \lambda} \int_{0}^{\tau_{v}}\left(a^{1 / 2}-\sigma\right)^{2} d t\right) .
\end{aligned}
$$

It is straightforward to show that the second part converges to

$$
\mathbb{E}^{\mathbb{P}}\left(\Phi\left(S_{T}^{a}\right)-\frac{1}{2 \lambda} \int_{0}^{T}\left(a^{1 / 2}-\sigma\right)^{2} d t\right)
$$

when $v$ goes to 0 , and this shows one part of (84). To show the other side (that $u$ indeed realizes the supremum), one considers again the stopped process and 
$a_{v, t}=\sigma^{\gamma} \mathbb{I}_{t \leq \tau_{v}}$. Then $S_{t}^{a_{v}}=S_{t \wedge \tau_{v}}, a_{v}$ belongs to $\mathcal{A}_{T}$, and the same arguments as above show that

$$
u\left(0, S_{0}\right)=\lim _{\nu \rightarrow 0} \mathbb{E}^{\mathbb{P}}\left(\Phi\left(S_{T}^{a_{\nu}}\right)-\frac{1}{2 \lambda} \int_{0}^{T}\left(a_{\nu}^{1 / 2}-\sigma\right)^{2} d t\right) .
$$

The statement about identity (40) is a direct consequence of (85).

6.3. Black-Scholes representation formula, and explicit formulation of the density. Going back to Section 5.3, we recall that $w=\left[v^{*}\right]^{-1}$, satisfies (53):

$$
\partial_{t} w+\frac{1}{2} \sigma^{2}\left(t, \frac{1}{\partial_{x} w}\right)\left(\partial_{x x} w+\partial_{x} w\right)=0 .
$$

Then, for $x=v^{*}\left(t, \partial_{s} v(t, s)\right)$,

$$
\begin{aligned}
w(t, x) & =\partial_{s} v(t, s) \\
& =-\frac{1}{s}-\lambda \partial_{s} u(t, s) \\
& =-\frac{1}{\mathcal{S}(t, x)}-\lambda \partial_{s} u(t, \mathcal{S}(t, x)),
\end{aligned}
$$

with $\mathcal{S}(t, x)=\frac{1}{\partial_{x} w}$; see Proposition 5.3, Point 7. We consider for $\tilde{\mathbb{P}}$ a probability on $\left(\Omega, \mathbb{F},\left(\mathcal{F}_{t}\right)_{t \geq 0}\right), W_{t}^{\tilde{\mathbb{P}}}$ a $\tilde{\mathbb{P}}$-Brownian motion and $\mathcal{Y}_{t}$ the solution to

$$
\begin{aligned}
d \mathcal{Y}_{t} & =\sigma\left(d W_{t}^{\tilde{\mathbb{P}}}+\sigma / 2 d t\right), \\
\sigma & =\sigma\left(t, \mathcal{S}\left(t, \mathcal{Y}_{t}\right)\right), \\
\mathcal{Y}_{0} & =V\left(0, S_{0}\right) .
\end{aligned}
$$

Under the assumptions of Theorem 5.12, $w$ has enough regularity to check that $w\left(t, \mathcal{Y}_{t}\right)$ is a local martingale on $[0, T)$. We have then the following representation result, which we call a modified Black-Scholes representation.

THEOREM 6.10 (Local volatility). Let u be a classical solution to (24), (25), (26), where $\sigma, \Phi$ satisfy the assumptions of Theorem 5.12. Let $w$ be defined from $u$ as above. Let $\mathcal{Y}_{t}$ be defined as in (93). Then $w\left(t, \mathcal{Y}_{t}\right)$ is a martingale up to time $T$,

$$
\begin{aligned}
-\frac{1}{S_{0}}-\lambda \partial_{s} u\left(t_{0}, S_{0}\right) & =w\left(t_{0}, \mathcal{Y}_{0}\right)=\mathbb{E}^{\tilde{\mathbb{P}}}\left(w\left(T, \mathcal{Y}_{T}\right)\right) \\
& =\mathbb{E}^{\tilde{\mathbb{P}}}\left(-\frac{1}{\mathcal{S}_{T}\left(\mathcal{Y}_{T}\right)}-\lambda \partial_{s} \Phi\left(\mathcal{S}_{T}\left(\mathcal{Y}_{T}\right)\right)\right)
\end{aligned}
$$

where $\mathcal{Y}_{0}$ is such that $\partial_{x} w\left(0, \mathcal{Y}_{0}\right)=\frac{1}{S_{0}}$, and then

$$
u\left(0, S_{0}\right)=S_{0} \partial_{s} u\left(0, S_{0}\right)+\frac{1}{\lambda}\left(\mathcal{Y}_{0}-\ln \left(S_{0}\right)\right) .
$$


If $u$ satisfies (32), and $s \partial_{s} \sigma$ is bounded, one can choose $\tilde{\mathbb{P}}$ absolutely continuous with respect to $\mathbb{P}$ such that $Y=\mathcal{Y} \mathbb{P}$-a.s., (where $Y$ has been defined in Proposition 6.5), and then

$$
d W_{t}^{\tilde{\mathbb{P}}}=d W_{t}^{\mathbb{P}}+\left(\frac{s \partial_{s} \sigma-\sigma}{1-\lambda \gamma} d t-s \partial_{s} \sigma\right) d t
$$

In particular, this holds true under the assumptions (i) to (v) of Theorem 5.8.

We state right away an extension of this result in the constant volatility case, which will come as a corollary of Theorems 6.6 and 6.10 .

THEOREM 6.11 (Constant volatility). In addition to the assumptions of Theorem 6.10, if $\sigma$ is constant and (82) holds, then:

1. One can chose $\tilde{\mathbb{P}}$ to be equal to $\mathbb{Q}$ defined in Proposition 6.5,

2. One can compute $u\left(0, S_{0}\right)$ as follows:

Step 1. From $\Phi$, compute $\mathcal{S}_{T}$ as stated in Proposition 5.3.

Step 2. Find $\mathcal{Y}_{0}$ such that

$$
\frac{1}{S_{0}}=\mathbb{E}^{\tilde{\mathbb{P}}}\left(\frac{1}{\mathcal{S}_{T}\left(\mathcal{Y}_{0}+\sigma W_{T}^{\tilde{\mathbb{P}}}+\frac{\sigma^{2}}{2} T\right)}\right) .
$$

Step 3. Compute

$$
u\left(0, S_{0}\right)=S_{0} \mathbb{E}^{\tilde{\mathbb{P}}}\left(\partial_{s} \Phi\left(\mathcal{S}_{T}\left(\mathcal{Y}_{0}+\sigma W_{T}^{\tilde{\mathbb{P}}}+\frac{\sigma^{2}}{2} T\right)\right)\right)+\frac{1}{\lambda}\left(\mathcal{Y}_{0}-\ln \left(S_{0}\right)\right)
$$

Moreover, there holds

$$
u\left(0, S_{0}\right)=\mathbb{E}^{\mathbb{P}}\left(S_{T} \partial_{s} \Phi\left(S_{T}\right)\right)-\frac{1}{\lambda}\left(\ln \left(S_{0}\right)-\mathcal{Y}_{0}\right) .
$$

3. For all $\varphi$ such that, $\int_{\mathbb{R}} \frac{\varphi\left(\mathcal{S}_{T}\right)}{\mathcal{S}_{T}} \exp \left(-\frac{y^{2}}{2 \sigma^{2} T}\right)$ is finite, there holds

$\mathbb{E}^{\mathbb{P}}\left(\varphi\left(S_{T}\right)\right)=S_{0} \mathbb{E}^{\tilde{\mathbb{P}}}\left(\frac{\varphi\left(\mathcal{S}_{T}\right)}{\mathcal{S}_{T}}\left(\sigma W_{T}^{\tilde{\mathbb{P}}}+\mathcal{Y}_{0}+\frac{\sigma^{2}}{2} T\right)\right)$

$$
=S_{0} \int_{\mathbb{R}} \varphi(s) \frac{1-\lambda s^{2} \partial_{s s} \Phi}{s^{2}} \exp \left(-\frac{\left(V_{T}(s)-\frac{\sigma^{2}}{2} T-\mathcal{Y}_{0}\right)^{2}}{2 \sigma^{2} T}\right) \frac{d s}{\sigma \sqrt{2 \pi T}} .
$$

4. If moreover $\int_{\mathbb{R}} \exp \left(-\frac{y^{2}}{2 \sigma^{2} T}\right) \frac{\ln \left(\mathcal{S}_{T}(y)\right)}{\mathcal{S}_{T}(y)} d y$ is finite, then

$$
\mathbb{E}^{\mathbb{P}}\left(\int_{0}^{T} \frac{d\langle S, S\rangle_{t}}{S_{t}^{2}}\right)=\mathbb{E}^{\mathbb{P}}\left(\int_{0}^{T} \frac{\sigma^{2}}{\left(1-\lambda \gamma\left(t, S_{t}\right)\right)^{2}}\right) \text { is finite, }
$$

and

$$
\mathbb{E}^{\mathbb{P}}\left(\Phi\left(S_{T}\right)\right) \text { is finite. }
$$


REMARKS. The formula (94) is a uniqueness result somehow reminiscent of Widder's theorem (see [22]): it shows that the solution of the parabolic equation (92) (seen as a linear equation) is unique and defined by the representation formula (94). Again the key ingredient here is that we restrict ourselves to concave solutions (which replaces the positivity assumption needed in Widder's theorem).

Regarding formulas (96) and why we call it a modified Black-Scholes formula, it is well known that the classical Black-Scholes formula for a call option consists of two terms:

$$
\begin{aligned}
u\left(0, S_{0}\right) & =S_{0} \mathcal{N}\left(d_{1}\right)-K \mathcal{N}\left(d_{0}\right) \\
& =S_{0} \partial_{s} u\left(0, S_{0}\right)-K \mathbb{P}\left\{S_{T} \geq K\right\} \\
& =\mathbb{E}\left(S_{T} \partial_{S} \Phi\left(S_{T}\right)\right)-\mathbb{E}\left(S_{T} \partial_{S} \Phi\left(S_{T}\right)-\Phi\left(S_{T}\right)\right) .
\end{aligned}
$$

Indeed, for $\left.\Phi=(S-K)^{+}, s \partial_{s} \Phi-\Phi=K \mathbb{I}_{S \geq K}\right)$. This last line is a tautology since in the Black-Scholes model, both $u\left(t, S_{t}\right)$ and $S_{t} \partial_{s} u\left(t, S_{t}\right)$ are martingales under the risk neutral probability. In the linear market impact model, there is a risk-neutral probability (the probability $\mathbb{P}$ in our notation), and while $u$ is not the expectation of its final value [see formula (40)], hence not a martingale under $\mathbb{P}$, when $\sigma$ is constant, $S_{t} \partial_{s} u\left(t, S_{t}\right)$ remains a martingale, and formula (96) reads similarly:

$$
u\left(0, S_{0}\right)=\mathbb{E}^{\mathbb{P}}\left(S_{T} \partial_{s} \Phi\left(S_{T}\right)\right)-\left(S_{0} \partial_{s} u\left(0, S_{0}\right)-u\left(0, S_{0}\right)\right)
$$

REMARK. Taking $w(t, x)=\zeta\left(t, e^{x}\right), \zeta$ satisfies

$$
\partial_{t} \zeta+\frac{\sigma^{2}(t, s)}{2} \xi^{2} \partial_{\xi \xi} \zeta+\sigma^{2} \xi \partial_{\xi} \zeta=0,
$$

with $s=\left(\xi \partial_{\xi} \zeta\right)^{-1}$. Then

$$
\zeta\left(t, s e^{-\lambda u^{c}(t, s)}\right)=-\frac{1}{s}-\lambda \partial_{s} u(t, s),
$$

with $u^{c}(t, s)=s \partial_{s} u-u$. We now let $\Xi_{t}$ be the solution to

$$
\begin{aligned}
\frac{d \Xi_{t}}{\Xi_{t}} & =\sigma\left(t, \mathcal{S}_{\lambda}\left(t, \ln \left(\Xi_{t}\right)\right)\right) d W_{t}^{\tilde{\mathbb{P}}}+\sigma^{2} d t, \\
\Xi_{t_{0}} & =S_{0} e^{-\lambda u^{c}\left(t_{0}, S_{0}\right)},
\end{aligned}
$$

and then $\zeta\left(t, \Xi_{t}\right)$ is a martingale, and a similar result holds.

Proof of THEOREM 6.10. The fact that $w\left(t, \mathcal{Y}_{t}\right)$ is a martingale up to time $T$ is proved in the Appendix, Lemma A.1.

To prove the existence of $\mathcal{Y}_{0}$, from Proposition 5.2, under Assumption 5.5 there holds: 
$-\lim _{\xi \rightarrow-\infty} \partial_{x} w_{T}(x)=+\infty$

$-\lim _{\xi \rightarrow+\infty} \partial_{x} w_{T}(x)=0$,

and $\partial_{x} w$ is nondecreasing, which allows to conclude the existence and uniqueness of $\mathcal{Y}_{0}$.

Proof of TheOREM 6.11. This theorem is a corollary of Theorems 6.6 and 6.10. For Point 1, once $S$ is a martingale up to $T$, one can define the probability $\mathbb{Q}$, and $Y$ follows then

$$
d Y_{t}=\sigma d W^{\mathbb{Q}}+\frac{\sigma^{2}}{2} d t
$$

for $W^{\mathbb{Q}}$ a $\mathbb{Q}$-Brownian motion.

For point 2, when $\sigma$ is constant, $\partial_{x} w$ follows also equation (53), which shows the Step 2 of the Point 2, and one then uses formula (94).

Point 3 is the direct consequence of (101).

Point 4 is a particular case of (97) when $\varphi(s)=\ln (s)$. Note that as remarked at the end of the proof of Theorem 6.6, the finiteness of $\mathbb{E}^{\mathbb{P}}\left(\ln \left(S_{T}\right)\right)$ is equivalent to the finiteness of $\mathbb{E}^{\mathbb{P}}\left(\Phi\left(S_{T}\right)\right)$.

7. A Black-Scholes-Legendre formula. In this section, we derive the firstorder expansion of the solution with respect to the market impact parmeter $\lambda$. To rigorously establish this expansion, we will need the regularity results of the pricing equation, and the representation formula previously established.

7.1. Formal computations. For this, we will use the identity (39). We are in the case where $\lambda, \sigma$ are constant. We still consider a probability $\mathbb{P}$ under which $S$ follows (34), (35), (36), and we let $\mathbb{Q}_{\mathrm{BS}}$ be a probability under which

$$
\frac{d S_{t}}{S_{t}}=\sigma d W_{t}^{\mathbb{Q B S}_{\mathrm{BS}}}
$$

for $W^{\mathbb{Q}} \mathbb{Q}_{\text {BS }}$ a $\mathbb{Q}_{\mathrm{BS}}$ Brownian motion. Consider $\bar{u}$ the Black and Scholes solution, that is, the solution to (24) for $\lambda=0$, a formal Taylor expansion around $\lambda=0$ of (24) yields

$$
v\left(0, S_{0}\right)=\bar{u}\left(0, S_{0}\right)+\frac{\lambda}{2} \mathbb{E}^{\mathbb{Q}_{\mathrm{BS}}}\left(\int_{0}^{T} \sigma^{2} \bar{\gamma}^{2} d t\right)+o(\lambda),
$$

and $\bar{\gamma}=s^{2} \partial_{s s} \bar{u}$. We now evaluate $\mathcal{I}=\mathbb{E}^{\mathbb{Q}_{\mathrm{BS}}}\left(\int_{0}^{T} \sigma^{2} \bar{\gamma}^{2} d t\right)$. To do this, we define

$$
\bar{u}^{c}(t, s)=s \partial_{s} \bar{u}-\bar{u},
$$

note that

$$
\bar{u}^{c}(t, s)=\bar{u}^{*}\left(t, \partial_{s} u(t, s)\right)
$$


for $\bar{u}^{*}$ the Legendre transform of $\bar{u}$. Observe that, if $\bar{u}$ solves the Black-Scholes equation, then $\bar{u}^{c}$ is a martingale under $\mathbb{Q}_{\mathrm{BS}}$ (this is checked by a simple computation), and that $d\left(\bar{u}^{c}\left(t, S_{t}\right)\right)=S_{t} \partial_{s s} \bar{u} d S_{t}$ under $\mathbb{Q}_{\mathrm{BS}}$ and, therefore,

$$
\begin{aligned}
\mathcal{J} & =\int_{0}^{T} \bar{\gamma} \sigma d W_{t}^{\mathbb{Q}_{\mathrm{BS}}} \\
& =\int_{0}^{T} S_{t} \partial_{s s} \bar{u} d S_{t} \\
& =\bar{u}^{c}\left(T, S_{T}\right)-\bar{u}^{c}\left(0, S_{0}\right) .
\end{aligned}
$$

Then

$$
\begin{aligned}
\mathcal{I} & =\mathbb{E}^{\mathbb{Q}_{\mathrm{BS}}}\left(\mathcal{J}^{2}\right) \\
& =\mathbb{E}^{\mathbb{Q}_{\mathrm{BS}}}\left(\bar{u}^{c}\left(T, S_{T}\right)-\bar{u}^{c}\left(0, S_{0}\right)\right)^{2} \\
& =\mathbb{E}^{\mathbb{Q}_{\mathrm{BS}}}\left(\bar{u}^{c}\left(T, S_{T}\right)^{2}\right)-\left(\bar{u}^{c}\left(0, S_{0}\right)\right)^{2} .
\end{aligned}
$$

We have thus obtained a first modified Black-Scholes-Legendre formula

$$
\begin{aligned}
u\left(0, S_{0}\right) & =\mathbb{E}^{\mathbb{Q}_{\mathrm{BS}}}(\Phi)+\frac{\lambda}{2}\left(\mathbb{E}^{\mathbb{Q}_{\mathrm{BS}}}\left(\left(\Phi^{c}\right)^{2}\right)-\left(\mathbb{E}^{\mathbb{Q}_{\mathrm{BS}}}\left(\Phi^{c}\right)\right)^{2}\right)+o(\lambda), \\
\Phi^{c} & =s \partial_{s} \Phi-\Phi .
\end{aligned}
$$

We then observe that, if $\Psi$ is any reasonable Lipschitz function and

$$
\Phi=\inf \left\{\Psi^{\prime} \geq \Psi, \lambda s^{2} \partial_{s s} \Psi^{\prime} \leq 1\right\},
$$

then

$$
\begin{aligned}
\mathbb{E}^{\mathbb{Q}_{\mathrm{BS}}}\left(\Phi\left(S_{T}\right)\right) & =\mathbb{E}^{\mathbb{Q}_{\mathrm{BS}}}\left(\Psi\left(S_{T}\right)\right)+O\left(\lambda^{2}\right), \\
\mathbb{E}^{\mathbb{Q}_{\mathrm{BS}}}\left(\Phi^{c}\left(S_{T}\right)\right) & =\mathbb{E}^{\mathbb{Q}_{\mathrm{BS}}}\left(\Psi^{c}\left(S_{T}\right)\right)+O(\lambda) .
\end{aligned}
$$

Hence, replacing $\Phi$ by $\Psi$ in the above formula will only add terms of order 2 in $\lambda$. In the case of a call (resp., put) option with strike $K$, we have $\Phi^{c}(s)=K \mathbb{I}_{s \geq K}$ [resp., $\left.\Phi^{c}(s)=-K \mathbb{I}_{s \leq K}\right]$. Now let

$$
d_{K}=\mathbb{E}^{\mathbb{Q} B S}\left(\mathbb{I}_{s \geq K}\right),
$$

$C_{\mathrm{BS}}$ (resp., $P_{\mathrm{BS}}$ ) be the Black-Scholes call (resp., put) price, and $C_{\mathrm{BS}}^{\lambda}\left(\right.$ resp., $\left.P_{\mathrm{BS}}^{\lambda}\right)$ the prices with market impact, then

$$
\begin{aligned}
& C_{\mathrm{BS}}^{\lambda}\left(0, S_{0}\right)=C_{\mathrm{BS}}\left(0, S_{0}\right)+\frac{\lambda}{2} K^{2}\left(d_{K}-d_{K}^{2}\right)+o(\lambda), \\
& P_{\mathrm{BS}}^{\lambda}\left(0, S_{0}\right)=P_{\mathrm{BS}}\left(0, S_{0}\right)+\frac{\lambda}{2} K^{2}\left(d_{K}-d_{K}^{2}\right)+o(\lambda) .
\end{aligned}
$$


REMARKS. Note that (103) allows to compute the first-order market impact correction using a simple Monte-Carlo/analytical pricer, for any terminal payoff as long as one is able to compute $\Phi^{c}$. It can be computed without relying on the constant volatility assumption, so it can still be computed in presence of local or even stochastic volatility; however, in those cases $u^{c}$ is not a martingale anymore, so the first-order expansion should be slightly different.

Note also that the analytical price of call spread with strikes $K_{1}<K_{2}$ would be easily computed since in that case $\Phi^{c}=K_{1} \mathbb{I}_{K_{1} \leq s \leq K_{2}}-\left(K_{2}-K_{1}\right) \mathbb{I}_{s \geq K_{2}}$.

Note that the correction term is indeed quadratic in $\Phi$.

The correction obtained here is also formally valid for the case of pure liquidity costs studied by Cetin, Soner and Touzi [8], since at first order the two pricing equations are the same, it also should be valid for any fully nonlinear modification of the Black-Scholes equation.

While it is relatively straightforward to make the arguments above rigorous in the case of a terminal payoff satisfying the constraint (33), it is much more involved without this assumption. This is what we establish in the next theorem. This result rests on the regularity results of Section 5 and on the representation formulas of Section 6.

\subsection{First-order expansion of the solution.}

THEOREM 7.1. For $\Psi: \mathbb{R}_{+}^{*} \rightarrow \mathbb{R}$, let $\Phi^{\lambda}$ be defined as in (104). Assume that $\Psi$ is globally Lipschitz and satisfies the assumptions (i) to (iv) of Theorem 5.8, and that $\Psi^{c}=s \partial_{s} \Psi-\Psi$ is bounded. Let $u^{\lambda}(t, s)$ be the solution of (24), (25), (26) with terminal condition $u=\Phi^{\lambda}$, with constant volatility parameter $\sigma$. Then $u$ is differentiable with respect to $\lambda$ and there holds

$$
\frac{\partial u^{\lambda}(t, s)}{\partial \lambda}=\mathbb{E}^{\mathbb{P}}\left(\int_{t}^{T} \frac{\sigma^{2}}{2} \frac{\gamma^{2}}{(1-\lambda \gamma)^{2}}\left(t, S_{r}^{t, s}\right) d t\right),
$$

where $S^{t, s}$ solves (34), (35), (36) and, moreover,

$$
\begin{aligned}
u^{\lambda}(t, s)= & \mathbb{E}^{\mathbb{Q}_{\mathrm{BS}}}\left(\Psi\left(S_{T}^{t, s}\right)\right) \\
& +\frac{\lambda}{2}\left(\mathbb{E}^{\mathbb{Q}_{\mathrm{BS}}}\left(\Psi^{c}\left(S_{T}^{t, s}\right)^{2}\right)-\left(\mathbb{E}^{\mathbb{Q}_{\mathrm{BS}}}\left(\Psi^{c}\left(S_{T}^{t, s}\right)\right)\right)^{2}\right)+o(\lambda) .
\end{aligned}
$$

PROOF. First, under our assumptions, and since $\sigma$ is constant, $u$ belongs to $C^{\infty}\left([0, T) \times \mathbb{R}_{+}^{*}\right)$, and moreover, we are under the assumptions of Theorem 6.6, hence there exists a unique global strong solution to (34), (35), (36) which is a martingale up to time $T$. We first start with a smoothed terminal payoff $\Psi^{\varepsilon}$ such that $-\varepsilon^{-1} \leq s^{2} \partial_{s s} \Psi^{\varepsilon} \leq \varepsilon^{-1}$. Then take $\Phi^{\lambda, \varepsilon}$ as in (104) but such that $1-\lambda s^{2} \partial_{s s} \Phi^{\lambda, \varepsilon} \geq \varepsilon$. Finally, $\Phi^{\lambda, \varepsilon}$ will satisfy

$$
\begin{gathered}
-\varepsilon^{-1} \leq s^{2} \partial_{s s} \Phi^{\lambda, \varepsilon} \leq \min \left\{\frac{1-\varepsilon}{\lambda}, \varepsilon^{-1}\right\}, \\
\Phi^{\lambda, \varepsilon} \rightarrow_{\varepsilon \rightarrow 0} \Phi^{\lambda} .
\end{gathered}
$$


From now on, we drop the superscripts $\lambda, \varepsilon$. Let $u_{\lambda}$ (resp., $\gamma_{\lambda}$ ) be the derivative of $u$ (resp., $\gamma$ ) with respect to $\lambda$. We choose $t=0, s=S_{0}$ and also drop the superscript $0, S_{0}$ for $S_{t}$. Then

$$
\partial_{t} u_{\lambda}+\frac{\sigma^{2}}{2} \frac{\gamma_{\lambda}}{(1-\lambda \gamma)^{2}}+\frac{\sigma^{2}}{2} \frac{\gamma^{2}}{(1-\lambda \gamma)^{2}}=0,
$$

and $u_{\lambda}(T)=\partial_{\lambda} \Phi^{\lambda}$ is supported on $\left\{\lambda s^{2} \partial_{s s} \Phi^{\lambda}=1\right\}$. One can thus write

$$
u_{\lambda}\left(0, S_{0}\right)=\mathbb{E}^{\mathbb{P}}\left(\int_{0}^{T} \frac{\sigma^{2}}{2} \frac{\gamma^{2}}{(1-\lambda \gamma)^{2}}\left(t, S_{t}\right) d t\right)+\mathbb{E}^{\mathbb{P}}\left(\partial_{\lambda} \Phi\left(S_{T}\right)\right) .
$$

$\partial_{\lambda} \Phi$ is supported on the set $\left\{\lambda s^{2} \partial_{s s} \Phi=1-\varepsilon\right\}$, that is, where $F\left(\gamma_{T}\right)=\varepsilon^{-1}$. From Theorem 6.3, we have that $\mathbb{P}\left\{F\left(\gamma_{T}\right) \geq \varepsilon^{-1}\right\} \leq C \varepsilon$, and since $\partial_{\lambda} \Phi$ is bounded, $\mathbb{E}^{\mathbb{P}}\left(\partial_{\lambda} \Phi\left(S_{T}\right)\right) \leq C \varepsilon$.

Under our assumptions and the regularity results of Theorems 5.8), (5.6, this integral is well defined for $\varepsilon>0$.

Then in order to show (107), we need to show that this integral indeed converges to the expected limit, as $\Psi^{\varepsilon}$ converges to $\Psi$. From Theorem 5.8, and given our assumptions on $\Psi$, we have uniform bounds on $\gamma$ and $(1-\lambda \gamma)^{-1}$ on $\left[0, T^{\prime}\right]$ for $T^{\prime}<T$. Hence the integral up to $T^{\prime}<T$ will converge when $\Psi^{\varepsilon}$ goes to $\Psi$ thanks to the dominated convergence theorem. We just need to show that

$$
\mathbb{E}^{\mathbb{P}}\left(\int_{T^{\prime}}^{T} \frac{\sigma^{2}}{2} \frac{\gamma^{2}}{(1-\lambda \gamma)^{2}}\left(t, S_{t}\right) d t\right)
$$

converges to 0 as $T^{\prime} \rightarrow T$, uniformly with respect to $\lambda, \varepsilon$ and to the terminal condition $\Psi$, as long as $\Psi$ satisfies the assumptions of the Theorem. For this, we will use the results of Theorem 6.6.

LEMMA 7.2. Under the assumptions of Theorem 7.1, let $\mathbb{Q}, Y$ be defined as in Proposition 6.7. For any smooth $C^{2}$ function $\phi(t, y)$ such that $S_{t} \phi\left(t, Y_{t}\right)$ is a martingale under $\mathbb{P}$ or $\phi\left(t, Y_{t}\right)$ is a martingale under $\mathbb{Q}$, there holds

$$
\partial_{t} \phi+\frac{\sigma^{2}}{2}\left(\partial_{y y} \phi+\partial_{y} \phi\right)=0 \text {. }
$$

In particular, this holds for

$$
\begin{aligned}
& \imath(t, y)=-\frac{1}{\mathcal{S}(t, y)}, \\
& J(t, y)=\partial_{s} u(t, \mathcal{S}(t, y)) .
\end{aligned}
$$

PROOF. Under the assumptions of Theorem 7.1, the assumptions of Theorem 6.6 are satisfied, hence $S$ is a martingale up to time $T$, and one can define $\mathbb{Q}$ as in Proposition 6.7. Then of course $S_{t} S_{t}^{-1}$ is a martingale under $\mathbb{P}$, 
hence $\left(\mathcal{S}\left(t, Y_{t}\right)\right)^{-1}$ is a martingale under $\mathbb{Q}$, and as shown in Theorem 6.10, $w=-\frac{1}{\mathcal{S}}-\lambda \partial_{s} u(\mathcal{S})$ is also a martingale under $\mathbb{Q}$, hence so is $\partial_{s} u(\mathcal{S})$.

We now observe that

$$
\begin{aligned}
\frac{\partial_{y} l}{l} & =-\frac{1}{1-\lambda \gamma}, \\
\frac{\gamma^{2}}{1-\lambda \gamma^{2}} & =\frac{1}{\lambda^{2}}\left(\frac{\partial_{y} l}{l}-1\right)^{2},
\end{aligned}
$$

and

$$
\partial_{y}(\imath+\lambda \jmath)=\imath
$$

We then compute

$$
\begin{aligned}
Q & =\mathbb{E}^{\mathbb{P}}\left(\int_{T^{\prime}}^{T} \frac{\sigma^{2}}{2} \frac{\gamma^{2}}{(1-\lambda \gamma)^{2}}\left(t, S_{t}\right) d t\right) \\
& =\mathbb{E}^{\mathbb{P}}\left(\int_{T^{\prime}}^{T} \frac{\sigma^{2}}{2} \frac{1}{\lambda^{2}}\left(\frac{\partial_{y} l}{\imath}-1\right)^{2}\left(t, Y_{t}\right) d t\right) \\
& =\mathbb{E}^{\mathbb{Q}}\left(\int_{T^{\prime}}^{T} \frac{\sigma^{2}}{2} \frac{-l^{-1}}{\lambda^{2}}\left(\partial_{y} l-\imath\right)^{2}\left(t, Y_{0}+\sigma W_{t}^{\mathbb{Q}}+\left(\sigma^{2} / 2\right) t\right) d t\right),
\end{aligned}
$$

where in the third line we have used formula (97). Then assuming that $\Psi^{c}$ is bounded, since $\imath=-\frac{1}{\mathcal{S}}$, and using the definition of $\mathcal{S}(55)$, there exists $C$ such that

$$
e^{-y-\lambda C} \leq l(T, y) \leq e^{-y+\lambda C},
$$

and this estimate is propagated by the heat equation, so that for another value of $C$ the bound (111) holds on $[0, T]$. Plugging this into the above equality yields

$$
\begin{aligned}
Q & \leq \mathbb{E}^{\mathbb{Q}}\left(\int_{T^{\prime}}^{T} \frac{C \sigma^{2}}{2 \lambda^{2}}\left(e^{y}\left(\partial_{y} \imath-\imath\right)^{2}\right)\left(t, Y_{0}+\sigma W_{t}^{\mathbb{Q}}+\left(\sigma^{2} / 2\right) t\right) d t\right) \\
& \leq \mathbb{E}^{\mathbb{Q}}\left(\frac{C^{\prime} \sigma^{2}}{2 \lambda^{2}} \int_{T^{\prime}}^{T}\left(\partial_{y} \imath-\imath\right)^{2}\left(t, Y_{0}+\sigma W_{t}^{\mathbb{Q}}+\left(3 \sigma^{2} / 2\right) t\right) d t\right),
\end{aligned}
$$

where we have used Girsanov's theorem for the second line. We now use (110) which yields for $j(t, y)=\partial_{s} u(t, \mathcal{S}(t, y))$

$$
Q \leq \mathbb{E}^{\mathbb{Q}}\left(\frac{C^{\prime} \sigma^{2}}{2} \int_{T^{\prime}}^{T}\left(\partial_{y} J\right)^{2}\left(t, Y_{0}+\sigma W_{t}^{\mathbb{Q}}+\left(3 \sigma^{2} / 2\right) t\right) d t\right),
$$

where $J$ solves (by Proposition 7.2)

$$
\partial_{t} J+\frac{\sigma^{2}}{2}\left(\partial_{y y} J+\partial_{y} J\right)=0 \text {. }
$$


Then it follows that, letting

$$
\begin{array}{r}
\tilde{Y}_{t}=Y_{0}+\sigma W_{t}^{\mathbb{Q}}+\left(3 \sigma^{2} / 2\right) t, \\
H_{T^{\prime}, T}=\int_{T^{\prime}}^{T} \partial_{y} J\left(t^{\prime}, \tilde{Y}_{t^{\prime}}\right) \sigma d W_{t}^{\mathbb{Q}},
\end{array}
$$

we have, for another constant $C$,

$$
Q \leq \mathbb{E}^{\mathbb{Q}}\left(C H_{T^{\prime}, T}^{2}\right) .
$$

Moreover, we have using (113)

$$
H_{T^{\prime}, T}=\jmath\left(T, \tilde{Y}_{T}\right)-\jmath\left(T^{\prime}, \tilde{Y}_{T^{\prime}}\right)-\int_{T^{\prime}}^{T} \partial_{y} \jmath\left(t, \tilde{Y}_{t}\right) \sigma^{2} d t,
$$

so that by Cauchy-Schwarz's inequality

$$
\left|H_{T^{\prime}, T}-\left(J\left(T, \tilde{Y}_{T}\right)-\jmath\left(T^{\prime}, \tilde{Y}_{T^{\prime}}\right)\right)\right| \leq \varepsilon\left(\int_{T^{\prime}}^{T}\left(\partial_{y} J\right)^{2}\left(t, \tilde{Y}_{t}\right) d t\right)^{1 / 2}
$$

for $\varepsilon=\sigma^{2}\left(T-T^{\prime}\right)^{1 / 2}$, hence, letting

$$
J_{T^{\prime}, T}=\jmath\left(T, \tilde{Y_{T}}\right)-\jmath\left(T^{\prime}, \tilde{Y_{T^{\prime}}}\right),
$$

we have, using (112),

$$
Q \leq C^{\prime \prime}\left(\mathbb{E}^{\mathbb{Q}}\left(J_{T^{\prime}, T}^{2}\right)+\varepsilon^{2} Q\right) .
$$

We now reintroduce the superscripts $\lambda, \varepsilon$ to account for the dependency of the solution with respect to those parameters. The conclusion of the proof comes now as follows: as $\lambda, \varepsilon, t \rightarrow \bar{\lambda}, \bar{\varepsilon}, \bar{t}$,

- $J^{\lambda, \varepsilon}(t, y)=\partial_{s} u^{\lambda, \varepsilon}\left(t, \mathcal{S}^{\lambda, \varepsilon}(t, y)\right)$ is bounded under our assumptions, and converges almost everywhere to $J^{\bar{\lambda}, \bar{\varepsilon}}(\bar{t}, y)$,

- therefore, by the dominated convergence theorem, for all $\bar{\lambda}, \bar{\varepsilon}$,

$$
\lim _{\left(\lambda, \varepsilon, T^{\prime}\right) \rightarrow(\bar{\lambda}, \bar{\varepsilon}, T)} \mathbb{E}^{\mathbb{Q}}\left(\left(J_{T^{\prime}, T}^{\lambda, \varepsilon}\right)^{2}\right)=0 .
$$

This implies that $Q^{\lambda, \varepsilon}\left(T^{\prime}, T\right)$ converges to 0 when $T^{\prime} \rightarrow T$ uniformly with respect to $\lambda, \varepsilon$, which concludes the proof of (107).

8. Numerical simulations. In this section, we present for illustration purposes a numerical implementation of the model. Another study of a numerical implementation scheme is provided in the companion paper [5]. We propose the following numerical scheme: We set $\varepsilon$ to a small constant $\left(\varepsilon=10^{-3}\right.$ in our applications), and divide the time interval $[0, T]$ into $N$ time intervals $\left[0, t_{1}, \ldots, t_{N}=T\right]$. We let $\Delta t=\frac{T}{N}$. 
- Define the truncated operator

- Initialize $i=N$.

$$
F^{\varepsilon}(\gamma, \bar{\gamma})=\frac{\gamma}{\max \{1-\lambda \bar{\gamma}, \varepsilon\}} .
$$

- Terminal condition Initialize $u\left(t_{N}\right)=\Phi(T)$.

- Time loop For $i=N$ down to $i=1$.

- Initialize $v_{1}=u\left(t_{i}\right)$.

- Nonlinear iterations for $j$ in $\left[1, \ldots, N_{\mathrm{itnl}}\right]$

* solve for $w$

$$
\frac{u\left(t_{i}\right)-w}{\Delta t}=-\frac{1}{2} \sigma^{2} F^{\varepsilon}\left(s^{2} \partial_{s s} w, s^{2} \partial_{s s} v_{j}\right),
$$

* set $v_{j+1}=w$ if $j<N_{\mathrm{itnl}}$ and do one more nonlinear iteration or

* set $u\left(t_{i-1}\right)=w$ and exit nonlinear iterations loop.

- Iterate on the time step $i-1$ with $u\left(t_{i-1}\right)$ set above.

Typically, the number of nonlinear iterations $N_{\mathrm{itnl}}$ needed for convergence was small: $N_{\text {itnl }}=3$ was enough in our numerical example.

For stability reasons, we use an implicit scheme in the nonlinear iterations. An alternative to this method would be to enforce the constraint on the gamma at each time step, but we empirically observe that our method worked quite well. As noticed above, with a constant volatility model, it is enough to enforce the upper bound on $\gamma$ on the terminal condition, but this fails to be true with a generic local volatility.

We present some numerical simulations of the linear model in Figures 3(a), 3(b), 3(c).

We present the cases of 100-strike put option Figure 3(a), minus a 100-strike put option Figure 3(b), and a 90-100 call spread, Figure 3(c). The numerical values used are $\lambda=5.0 \times 10^{-3}, \sigma=0.3$, no interest rates or dividends. Note that in Figure 3(a) [resp., Figure 3(b)] the option sold is convex (resp., concave), while in Figure 3(c) the second derivative of the payoff changes sign. One sees that in all cases the market impact plays against the option's seller (the put sold is more expensive, and put bought is cheaper).

\section{APPENDIX A: PROOF OF PROPOSITIONS 5.9 AND 5.10}

A.1. Preliminary lemmas. The proofs rest on a barrier argument which is established in Lemmas A.1, A.2.

Lemma A.1. Let $w_{T}$ be concave and nondecreasing. Assume that $\sigma \in C_{t, s, \text { loc }}^{1,0}$ such that $\underline{\sigma} \leq \sigma(t, s) \leq \bar{\sigma}$ and let $\bar{w}, \underline{w}$ be defined by

$$
\bar{w}(t, x)=\frac{1}{\bar{\sigma} \sqrt{2 \pi(T-t)}} \int_{\mathbb{R}} \exp \left(-\frac{\left(x+\frac{1}{2} \underline{\sigma}^{2}(T-t)-z\right)^{2}}{2 \bar{\sigma}^{2}(T-t)}\right) w_{T}(z) d z,
$$




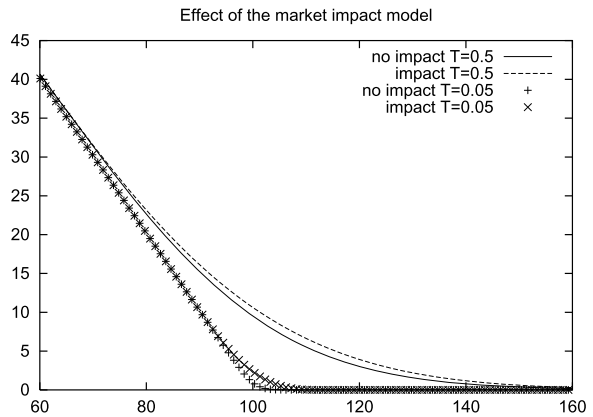

(a) The case a put option (gamma short case)

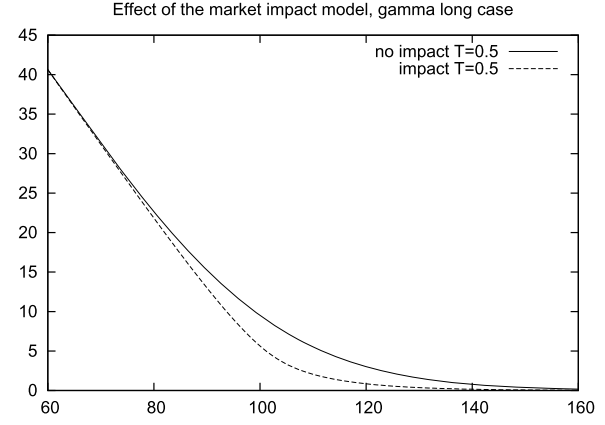

(b) The case of a put option(gamma long case)

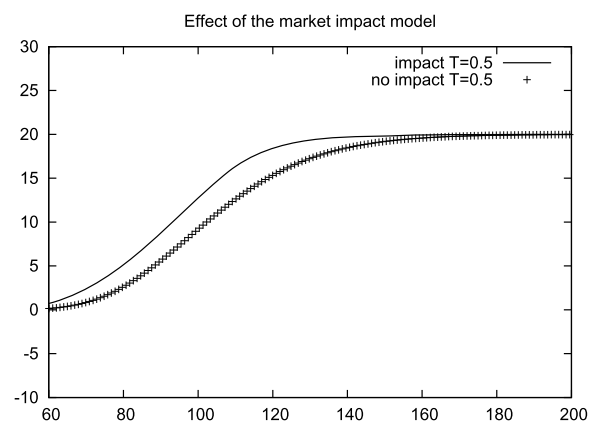

(c) The call spread case

FIG. 3. Numerical simulations.

$$
\underline{w}(t, x)=\frac{1}{\underline{\sigma} \sqrt{2 \pi(T-t)}} \int_{\mathbb{R}} \exp \left(-\frac{\left(x+\frac{1}{2} \bar{\sigma}^{2}(T-t)-z\right)^{2}}{2 \underline{\sigma}^{2}(T-t)}\right) w_{T}(z) d z,
$$

and assume that $\bar{w}, \underline{w}$ are both finite on $[-\varepsilon, T]$ for some $\varepsilon>0$. Let $w$ be a $C_{x, t, \mathrm{loc}}^{2,1}([0, T) \times \mathbb{R}) \cap C([0, T] \times \mathbb{R})$ classical solution to

$$
\begin{aligned}
\partial_{t} w+\frac{1}{2} \sigma^{2}\left(t, 1 / \partial_{x} w\right)\left(\partial_{x x} w+\partial_{x} w\right) & =0, \\
w(T, \cdot) & =w_{T}(\cdot),
\end{aligned}
$$

such that $\partial_{x x} w \leq 0, \partial_{x} w>0$ on $[0, T)$. Let $\mathcal{Y}_{r}^{t, x}$ be the unique strong solution to

$$
\begin{aligned}
d \mathcal{Y}_{r}^{t, x}= & \sigma\left(r, 1 / \partial_{x} w\left(r, \mathcal{Y}_{r}^{t, x}\right)\right) d W_{r}^{\mathbb{P}} \\
& +\frac{1}{2} \sigma^{2}\left(r, 1 / \partial_{x} w\left(r, \mathcal{Y}_{r}^{t, x}\right)\right) d r, \quad r \in[t, T], \\
\mathcal{Y}_{t}^{t, x}= & x .
\end{aligned}
$$

Then $w\left(r, \mathcal{Y}_{r}^{t, x}\right)$ is a martingale up to time $T$ and, moreover,

$$
\bar{w} \leq w \leq \underline{w} .
$$


In particular, if $u$ is a classical solution to (24), (25), (26) on [0,T), and $w$ is obtained from $u$ through the procedure of Section 5.3, then the conclusion holds true.

It is a simple comparison principle, but is not trivial, since following some famous counterexamples by Tychonoff (see [38]) uniqueness and comparison for solutions of the heat equation on the whole line do not hold unless some growth conditions are imposed. Here, we do not need any growth but we use the concavity of the solution, hence this result can be seen as a Widder's-type theorem (see [22]) which states uniqueness of positive solutions to the heat equation.

Using Lemma A.1, we construct two barriers for the solution.

LEMMA A.2. Under the assumptions of Theorem 5.8, one can find another constant $A^{\prime}$ instead of $A$ such that the properties (i) and (ii) are satisfied by the solution $u$ on $[0, T]$.

A.2. Proof of Proposition 5.9. We deduce Proposition 5.9 as a corollary of Lemma A.1. Starting from $\bar{w}$ which satisfies

$$
\partial_{t} \bar{w}+\frac{1}{2} \bar{\sigma}^{2} \partial_{x x} \bar{w}+\frac{1}{2} \sigma^{2} \partial_{x} \bar{w}=0,
$$

we perform in reverse order the operations that lead from $u$ to $w$, as described in Proposition 5.3: we first take the inverse of $w$ and obtain $\bar{v}^{*}$ which satisfies

$$
\partial_{t} \bar{v}^{*}+\frac{1}{2} \bar{\sigma}^{2} \frac{\partial_{y y} \bar{v}^{*}}{\left(\partial_{y} \bar{v}^{*}\right)^{2}}=\frac{1}{2} \underline{\sigma}^{2} .
$$

Then, taking $\bar{v}$ the Legendre transform of $\bar{v}^{*}$, and then $\bar{u}_{1}=-\lambda^{-1}(\ln (s)+\bar{v}+1)$ we obtain that $\bar{u}_{1}$ satisfies

$$
\partial_{t} \bar{u}_{1}+\frac{1}{2} \bar{\sigma}^{2} \frac{s^{2} \partial_{s s} \bar{u}_{1}}{1-\lambda s^{2} \partial_{s s} \bar{u}_{1}}=\frac{1}{2}\left(\underline{\sigma}^{2}-\bar{\sigma}^{2}\right) .
$$

Then $\bar{u}=\bar{u}_{1}+\frac{1}{2}(T-t)\left(\underline{\sigma}^{2}-\bar{\sigma}^{2}\right)$ satisfies (24), (25), (26) with $\sigma=\bar{\sigma}$. We construct $\underline{u}_{1}, \underline{u}$ in a similar way. By construction, we have

$$
\begin{aligned}
\bar{w} \leq w \leq \underline{w} & \Leftrightarrow \quad \underline{v}^{*} \leq v^{*} \leq \bar{v}^{*} \\
& \Leftrightarrow \quad \bar{v} \leq v \leq \underline{v} \\
& \Leftrightarrow \quad \underline{u}_{1} \leq v \leq \bar{u}_{1} \\
& \Leftrightarrow \quad \underline{u}-\frac{1}{2}(T-t)\left(\bar{\sigma}^{2}-\underline{\sigma}^{2}\right) \leq u \leq \bar{u}+\frac{1}{2}(T-t)\left(\bar{\sigma}^{2}-\underline{\sigma}^{2}\right),
\end{aligned}
$$

and this concludes the proof of Proposition 5.9. 
A.3. Proof of Proposition 5.10. Before proving Lemmas A.1 and A.2, we prove points (ii) and (iii) of Proposition 5.10. We first observe that

$$
(-\lambda \varepsilon \ln (s)+a+b s)^{*}=\lambda \varepsilon(\ln (\lambda \varepsilon)-1-\ln (b-y))-a .
$$

We focus first on the behaviour of $u$ when $s \rightarrow+\infty$, which means that for $y$, the argument of $v^{*}, y \rightarrow-\lambda B$. The estimate (ii) of Theorem 5.8 combined with Lemma A.2 and (120) yield that for $y, y^{\prime}$ large close enough to $-\lambda B$

$$
v^{*}\left(y^{\prime}\right)-v^{*}(y)=\lambda \varepsilon_{2} \ln \left(\frac{\lambda B+y}{\lambda B+y^{\prime}}\right)+Q\left(y, y^{\prime}\right),
$$

where $|Q| \leq 2 \lambda A$. Moreover, by the convexity of $v^{*}$,

$$
\partial_{y} v^{*}(y)\left(y^{\prime}-y\right) \leq v^{*}\left(y^{\prime}\right)-v^{*}(y) \leq \partial_{y} v^{*}\left(y^{\prime}\right)\left(y^{\prime}-y\right) .
$$

For $y^{\prime} \geq y$, we therefore have

$$
\begin{aligned}
& \partial_{y} v^{*}(y) \leq \frac{1}{y^{\prime}-y}\left(\lambda \varepsilon_{2} \ln \left(\frac{\lambda B+y}{\lambda B+y^{\prime}}\right)+Q\left(y, y^{\prime}\right)\right), \\
& \partial_{y} v^{*}\left(y^{\prime}\right) \geq \frac{1}{y^{\prime}-y}\left(\lambda \varepsilon_{2} \ln \left(\frac{\lambda B+y}{\lambda B+y^{\prime}}\right)+Q\left(y, y^{\prime}\right)\right) .
\end{aligned}
$$

We choose

$$
y^{\prime}-y=-R\left(\lambda B+y^{\prime}\right)
$$

and we have

$$
\begin{gathered}
y^{\prime}-y=-\frac{R}{1+R}(\lambda B+y), \\
\lambda B+y=(1+R)\left(\lambda B+y^{\prime}\right) .
\end{gathered}
$$

First fixing $y$ and moving $y^{\prime}$, or fixing $y^{\prime}$ and moving $y$, by (124) one can take $R$ large enough, but controlled by constants depending on $\lambda, \varepsilon_{2}, A$, so that

$$
\lambda \varepsilon_{2} \ln \left(\frac{\lambda B+y}{\lambda B+y^{\prime}}\right)+Q\left(y^{\prime}, y\right)=\varepsilon_{0}\left(\lambda, \varepsilon_{2}, A\right)>0
$$

and then

$$
\begin{gathered}
\partial_{y} v^{*}(y) \leq \frac{\varepsilon_{0}}{y^{\prime}-y}, \\
\partial_{y} v^{*}\left(y^{\prime}\right) \geq \frac{\varepsilon_{0}}{y^{\prime}-y} .
\end{gathered}
$$

By (122), (123), this implies that there exists $\theta_{1}, \theta_{2}>0$ such that

$$
\frac{\theta_{1}}{-(y+\lambda B)} \leq \partial_{y} v^{*} \leq \frac{\theta_{2}}{-(y+\lambda B)}
$$


as in the first claim of Lemma A.2. The proof for $s \rightarrow 0$ follows the same lines.

Then, for $\alpha, \theta>0$,

$$
\frac{1}{\partial_{y} v^{*}(-\lambda B-\alpha \theta)} \geq \frac{\alpha \theta}{\theta_{2}},
$$

while

$$
\frac{1}{\partial_{y} v^{*}(-\lambda B-\alpha)} \leq \frac{\alpha}{\theta_{1}}
$$

Therefore,

$$
\frac{1}{\partial_{y} v^{*}(-\lambda B-\alpha)}-\frac{1}{\partial_{y} v^{*}(-\lambda B-\alpha \theta)} \leq \alpha \frac{\theta_{2}-\theta \theta_{1}}{\theta_{1} \theta_{2}}
$$

Choosing $\theta=\frac{\theta_{2}}{\theta_{1}}+\theta_{2}$, we have that

$$
\frac{1}{\partial_{y} v^{*}(-\lambda B-\alpha \theta)} \geq \frac{1}{\partial_{y} v^{*}(-\lambda B-\alpha)}+\alpha,
$$

and this achieves the proof of Proposition 5.10.

A.4. Proof of Lemma A.1. We consider $\mathcal{Y}^{t, x}$ defined in (116), (117) under the probability $\tilde{\mathbb{P}}$, with $W^{\tilde{\mathbb{P}}}$ a $\tilde{\mathbb{P}}$-Brownian motion, and $\mathbb{E}^{\tilde{\mathbb{P}}}$ the expectation under $\tilde{\mathbb{P}}$. We drop the superscripts $\tilde{\mathbb{P}}$ over $W_{t}$ and $t, x$ over $\mathcal{Y}$ for the rest of the proof. We assume $w \in C_{s, t, \text { loc }}^{2,1}\left([0, T) \times \mathbb{R}_{+}^{*}\right)$, hence $w$ has enough regularity so that there exists a unique strong solution to (116), (117). First, note that $w$ is concave, increasing and $\partial_{x} w$ has limit equal to 0 at $+\infty$. Then since $\sigma \leq \bar{\sigma}$,

$$
\frac{d}{d t}\left(w\left(t, x+\frac{1}{2} \bar{\sigma}^{2}(t-T)\right)\right)=-\frac{1}{2} \sigma^{2} \partial_{x x} w+\frac{1}{2}\left(\bar{\sigma}^{2}-\sigma^{2}\right) \partial_{x} w \geq 0,
$$

hence $t \rightarrow w\left(t, x+\frac{1}{2} \bar{\sigma}^{2}(t-T)\right)$ is nondecreasing. The concavity of $w$ implies then that

$$
w\left(t, x+\frac{1}{2} \bar{\sigma}^{2}(t-T)\right)-w_{T}(0)-x \partial_{x} w_{T}(0) \leq 0 .
$$

Then, following [16], Theorem 9, Chapter 2, Section 4, there exists a unique solution to (53) such that $w^{+} \leq C e^{c|x|^{2}}$. From (127), $w$ must coincide with this solution.

Then considering

$$
\tilde{w}(t, x)=\mathbb{E}\left(w\left(T, \mathcal{Y}_{T}^{t, x}\right)\right),
$$

we have first by the monotonicity and concavity of $w_{T}$ that

$$
\begin{aligned}
& \mathbb{E}\left(w\left(T, x+\bar{\sigma}\left(W_{T}-W_{t}\right)+\frac{1}{2} \underline{\sigma}^{2}(T-t)\right)\right) \\
& \quad \leq \tilde{w}(t, x) \leq \mathbb{E}\left(w\left(T, x+\underline{\sigma}\left(W_{T}-W_{t}\right)+\frac{1}{2} \bar{\sigma}^{2}(T-t)\right)\right),
\end{aligned}
$$


which means that

$$
\bar{w} \leq \tilde{w} \leq \underline{w} .
$$

Therefore, $\tilde{w}$ is well defined. As $\underline{w}$ satisfies (127), so does $\tilde{w}$, and it is solution to (53). It must therefore coincide with $w$. This completes the proof of Lemma A.1.

A.5. Proof of Lemma A.2. From the definition of $v_{T}, v_{T}^{*}$ in Proposition 5.3, and using (120), we obtain by direct computations that the assumptions on $\Phi$ imply for $v_{T}^{*}$ that, for some constant $C, N$ depending on the constants in the assumptions (i), (ii) of Theorem 5.8:

(i) For $y \leq-N$,

$$
-\lambda \varepsilon_{1} \ln (-(y+\lambda B))-\lambda C \leq v_{T}^{*} \leq-\lambda \varepsilon_{1} \ln (-(y+\lambda B))+\lambda C .
$$

(ii) For $y \geq-\lambda B-1 / N$,

$$
-\lambda \varepsilon_{2} \ln (-(y+\lambda B))-\lambda C \leq v_{T}^{*} \leq-\lambda \varepsilon_{2} \ln (-(y+\lambda B))+\lambda C .
$$

These controls on $v_{T}^{*}$ are equivalent to the following controls on $w_{T}$ (the inverse function of $v_{T}^{*}$ ):

- For $x$ negative and large enough,

$$
s-\exp \left(-\frac{x-\lambda C}{\lambda \epsilon_{1}}\right) \leq w_{T}+\lambda B \leq-\exp \left(-\frac{x+\lambda C}{\lambda \epsilon_{1}}\right) .
$$

- For $x$ positive and large enough,

$$
-\exp \left(-\frac{x-\lambda C}{\lambda \epsilon_{2}}\right) \leq w_{T}+\lambda B \leq-\exp \left(-\frac{x+\lambda C}{\lambda \epsilon_{2}}\right) .
$$

We consider $\bar{w}, \underline{w}$ in Lemma A.1, $\bar{v}^{*}, \underline{v}^{*}$ their inverse functions. Lemma A.1 implies then that

$$
\underline{v}^{*} \leq v^{*} \leq \bar{v}^{*}
$$

for $t \in[0, T]$.

By the definitions of $\bar{w}, \underline{w}(114),(115)$, elementary computations on the heat kernel show that properties (130), (131) are satisfied by $\bar{w}(t), \underline{w}(t)$ for $t \in[0, T]$ with a different constant $C$ that depends on $T$. Since $\bar{v}^{*}, \underline{v}^{*}$ are the inverse of $\bar{w}, \underline{w}$, it follows that $\bar{v}^{*}, \underline{v}^{*}$ satisfy (128), (129) with a constant $C$ that also depends on $\bar{T}$. Therefore, $v^{*}$ being pinched between $\bar{v}^{*}, \underline{v}^{*}$ also satisfies (128), (129). Taking the Legendre transform of $v^{*}$ to get back to $v$, we find the result of Lemma A.2. 


\section{APPENDIX B: PROOF OF THEOREM 5.12}

Under the condition $\lambda s^{2} \partial_{s s} \Phi \leq 1, \lim _{s \rightarrow \infty} \partial_{s} \Phi=\mathcal{L} \in \mathbb{R} \cup\{-\infty\}$. We first prove the following lemma.

LEMMA B.1. Let $\Phi$ satisfy Assumption (5.5). For all $\tau>0$, for all $\kappa^{\prime}>0$ small, there exists $(K, \kappa)\left(\tau, \kappa^{\prime}\right)$, such that for $t^{\prime} \in[0, T-\tau]$,

$$
\left[\kappa^{\prime}, 1 / \kappa^{\prime}\right] \subset \partial_{y} v^{*}\left(t^{\prime},\left[-K, K_{\mathcal{L}}\right]\right) \subset[\kappa, 1 / \kappa],
$$

where

$$
K_{\mathcal{L}}= \begin{cases}\mathcal{L}-\frac{1}{K} & \text { if } \mathcal{L} \text { is finite }, \\ K & \text { otherwise. }\end{cases}
$$

The constants $K, \kappa$ depend only on the behaviour at $-\infty$ and $+\infty$ of $w_{T}$ defined in Proposition 5.3.

PROOF. We start by constructing two barriers as in the proof of Theorem 5.8: $\bar{v}^{*}, \underline{v}^{*}$ are the inverse of $\bar{w}, \underline{w}$ defined by (114), (115) in Lemma A.1, and $\bar{w} \leq$ $w \leq \underline{w}$, which then yields $\underline{v}^{*} \leq v^{*} \leq \bar{v}^{*}$. From the construction of $w_{T}$ (see Proposition 5.3, point 8), under Assumption (5.5) there holds

$$
\begin{array}{r}
\lim _{s \rightarrow 0} \ln (s)+\lambda\left(\Phi-s \partial_{s} \Phi\right)=-\infty, \\
\lim _{s \rightarrow 0}-\frac{1}{s}-\lambda \partial_{s} \Phi=-\infty
\end{array}
$$

and, moreover,

$$
\begin{aligned}
w_{T}\left(\ln (s)+\lambda\left(\Phi-s \partial_{s} \Phi\right)\right) & =-\frac{1}{s}-\lambda \partial_{s} \Phi, \\
\partial_{x} w_{T}\left(\ln (s)+\lambda\left(\Phi-s \partial_{s} \Phi\right)\right) & =\frac{1}{s},
\end{aligned}
$$

which imply that

$$
\begin{aligned}
\lim _{x \rightarrow-\infty} \partial_{x} w_{T} & =+\infty, \\
\lim _{x \rightarrow+\infty} \partial_{x} w_{T} & =0, \\
\lim _{x \rightarrow+\infty} w_{T} & =-\lambda \mathcal{L} \in \mathbb{R} \cup+\infty .
\end{aligned}
$$

Limits (132), (133), (134) are propagated for time $t \leq T$ by the definition (115), (114), and, for $t<T, \bar{w}$ and $\underline{w}$ are strictly increasing. Moreover, as noted in (126), for all $x, t \rightarrow w\left(t, x+\frac{\bar{\sigma}^{2}}{2} t\right)$ is nondecreasing (and the same holds for $\bar{w}, \underline{w}$ ), hence

$$
\bar{w}\left(0, x-\frac{\bar{\sigma}^{2}}{2} t\right) \leq w(t, x) \leq \underline{w}\left(T-\tau, x+\frac{\bar{\sigma}^{2}}{2}(T-\tau-t)\right) .
$$


The concavity of $w$ and (132), (133), (134) imply then a control on the way in which $\partial_{x} w$ goes to $-\infty$ at $-\infty$ and goes to 0 at $+\infty$, which passing to $\partial_{y} v^{*}=$ $1 / \partial_{x} w$ yields the lemma.

Proof of TheOREM 5.12. We study (52) on compact sets of $(\infty,-\lambda \mathcal{L}) \times$ $[0, T-\tau]$ for small $\tau$. From Lemma B.1, we see that (52) is uniformly parabolic and that we have an a priori bound for $v^{*}$ and $\partial_{y} v^{*}$. We can thus apply Lemma 5.11, to obtain local $C_{x}^{1+\alpha}$ regularity for $v^{*}$ for time $t<T$. As in the proof of Theorem 5.8, equation (52) on $v^{*}$ can now be seen as a linear parabolic equation with Hölder coefficients, and Schauder estimates then yield that $v^{*} \in C_{t, y, \operatorname{loc}}^{1+\alpha / 2,2+\alpha}([0, T) \times(-\infty,-\lambda \mathcal{L}))$. By differentiating the equation, further regularity follows if $\sigma$ has additional regularity. Then using the left-hand side of the inequality in Lemma B.1, the local regularity of $v^{*}$ on $(-\infty,-\lambda \mathcal{L})$ implies local regularity of $v$ on $\mathbb{R}_{+}^{*}$, henceforth of $u$ on any compact set of $[0, T) \times \mathbb{R}_{+}^{*}$.

For the last point where we require only Hölder continuity for $\sigma$, we remark that by differentiating equation (53), $\partial_{x} w$ follows a uniformly parabolic equation in divergence form, and hence following [25], Theorem 6.28, is Hölder continuous. Then $\partial_{y} v^{*}$ is also Hölder continuous, and the rest of the proof is the same.

This achieves the proof of Theorem 5.12.

\section{APPENDIX C: PROOF OF THEOREM 5.16}

We perform the change of variable $s=e^{y}$, and consider $v_{i}(t, y)=u_{i}\left(t, e^{y}\right), i=$ 1,2 , which yields that $s^{2} \partial_{s s} u_{i}=\partial_{y y} v_{i}-\partial_{y} v_{i}$. Under the assumptions of Theorem 5.16, $u_{1}$ and $u_{2}$ have logarithmic growth near 0 and linear growth at $+\infty$, hence $v_{1}, v_{2}$ have exponential growth. The derivatives of $v_{i}$ with respect to $y$ are bounded up to order 3, and $\sigma$ is uniformly Lipschitz as a function of $y$. Then $w=v_{1}-v_{2}$ solves

$$
\partial_{t} w+\frac{\sigma^{2}\left(t, e^{y}\right)}{2} \frac{\partial_{y y} w-\partial_{y} w}{\left(1-\lambda\left(\partial_{y y} v_{1}-\partial_{y} v_{1}\right)\right)\left(1-\lambda\left(\partial_{y y} v_{2}-\partial_{y} v_{2}\right)\right)}=0
$$

This can be seen as a linear equation of the form

$$
\partial_{t} w+a(t, y) \partial_{y y} w+b(t, y) \partial_{y} w=0
$$

where $a, b$ are bounded, uniformly Lipschitz in $y$ and $a$ bounded away from 0 . Then we can apply classical results of comparison under exponential growth (see [28]).

\section{APPENDIX D: EXISTENCE OF SMOOTH SOLUTIONS FOR GENERAL $\lambda$}

Here we state a simple result of smoothness for "good" initial data in the case where $\lambda=\lambda(\gamma)$, when $\sigma$ is constant. In this case, we need to start with well behaved solutions, as the singularity of the solution cannot be treated by the Legendre transform technique of the previous section. What we show is the following. 
THEOREM D.1. Let the final payoff $\Phi$ satisfy

$$
-\frac{1}{\varepsilon} \leq \lambda s^{2} \partial_{s s} \Phi \leq 1-\varepsilon
$$

for some $\varepsilon>0$. Assume that $\lambda(\gamma)$ is smooth satisfies locally uniformly with respect to $\gamma$ the condition (30). Then there exists a smooth solution to (24), (25), (26) such that (135) is satisfied for all time, that belongs to $C_{s, t}^{2+\alpha, 1+\alpha / 2}\left([0, T) \times \mathbb{R}_{+}^{*}\right)$.

PROOF. We only sketch the proof. This is an adaptation of Proposition 5.14 that relies on the estimate formula (77). Using the conditions on the boundary data $\Phi$, one can, using the continuity method (cf. Proposition 5.14), construct a solution such that (77) holds and $\gamma$ remains bounded above and below. Then classical parabolic regularity theory yields the result.

We make the following observation.

Proposition D.2. Let $0 \in I, F: \gamma \in I \rightarrow \mathbb{R}$ be a smooth increasing function such that $F(0)=0$ and $F(\gamma) \geq \gamma$. Then there exists $\lambda(\gamma) \geq 0$ such that

$$
F(\gamma)=\frac{\gamma}{1-\lambda(\gamma) \gamma}
$$

Moreover, $\lambda=\frac{1}{\gamma}-\frac{1}{F}$.

This comes by elementary computations, and shows that under mild conditions on $F$, any fully nonlinear pde of the form $\partial_{t} u+\frac{1}{2} \sigma^{2} F\left(s^{2} \partial_{s s} u\right)=0$ can be derived as a market impact pricing equation. Note that the conditions imply $F^{\prime}(0)=1$ and $F^{\prime \prime}(0) \geq 0$.

Acknowledgements. This paper has been in gestation for a long time, its motivation originally appeared while the author was a member of the Quantitative Research team at BNP Paribas Global Markets, and I thank my colleagues there for stimulating discussions. Part of this work has also been done under the hospitality of the Chair of Quantitative Finance at the Ecole Centrale de Paris, and I thank in particular Frédéric Abergel. I also thank Bruno Bouchard, Nizar Touzi, Juan Vazquez, Fernando Quiros, Ben Andrews and Fima Klebaner for enlightening discussions.

\section{REFERENCES}

[1] Abergel, F. and Loeper, G. (2016). Option pricing and hedging with liquidity costs and market impact. In Econophysics and Sociophysics: Recent Progress and Future Directions (F. Abergel, H. Aoyama, B. K. Chakrabarti, A. Chakraborti, N. Deo, D. Raina and I. Vodenska, eds.) 19-40. Springer, Cham.

[2] Almgren, R. A. (2005). Equity market impact. RISK 57-62. 
[3] Barles, G. (1994). Solutions de Viscosité des équations de Hamilton-Jacobi. Mathématiques \& Applications [Mathematics \& Applications] 17. Springer, Paris.

[4] Bouchard, B., Loeper, G. and Zou, Y. (2016). Almost-sure hedging with permanent price impact. Finance Stoch. 20 741-771. MR3519167

[5] Bouchard, B., Loeper, G. and Zou, Y. (2017). Hedging of covered options with linear market impact and gamma constraint. SIAM J. Control Optim. 55 3319-3348. MR3715374

[6] BRENIER, Y. (1991). Polar factorization and monotone rearrangement of vector-valued functions. Comm. Pure Appl. Math. 44 375-417. MR1100809

[7] Çetin, U., Jarrow, R. A. and Protter, P. (2004). Liquidity risk and arbitrage pricing theory. Finance Stoch. 8 311-341. MR2213255

[8] Çetin, U., Soner, H. M. and TouzI, N. (2010). Option hedging for small investors under liquidity costs. Finance Stoch. 14 317-341. MR2670416

[9] Cheridito, P., Mete Soner, H., Touzi, N. and Victoir, N. (2007). Second-order backward stochastic differential equations and fully nonlinear parabolic PDEs. Comm. Pure Appl. Math. 60 1081-1110.

[10] Cheridito, P., Soner, H. M. and Touzi, N. (2005). The multi-dimensional superreplication problem under gamma constraints. Ann. Inst. H. Poincaré Anal. Non Linéaire 22 633-666. MR2171995

[11] Crandall, M. and Pierre, M. (1982). Regularizing effects for $u_{t}+A \varphi(u)=0$ in $L^{1}$. J. Funct. Anal. 45 194-212.

[12] Crandall, M. G., Ishit, H. and Lions, P.-L. (1992). User's guide to viscosity solutions of second order partial differential equations. Bull. Amer. Math. Soc. (N.S.) 27 1-67. MR1118699

[13] Crandall, M. G. and Pierre, M. (1982). Regularizing effects for $u_{t}=\Delta \varphi(u)$. Trans. Amer. Math. Soc. 274 159-168. MR0670925

[14] FREY, R. Perfect option hedging for a large trader. Finance Stoch. 2 115-141.

[15] Frey, R. and Stremme, A. (1997). Market volatility and feedback effects from dynamic hedging. Math. Finance 7 351-374. MR1482708

[16] Friedman, A. (1964). Partial Differential Equations of Parabolic Type. Prentice-Hall, Englewood Cliffs, NJ.

[17] Friedman, A. (1975). Stochastic Differential Equations and Applications, Vol. 1. Probability and Mathematical Statistics 28. Academic Press, New York. MR0494490

[18] Gilbarg, D. and Trudinger, N. S. (1983). Elliptic Partial Differential Equations of Second Order, 2nd ed. Grundlehren der Mathematischen Wissenschaften [Fundamental Principles of Mathematical Sciences] 224. Springer, Berlin.

[19] Hsu, E. P. (2002). Stochastic Analysis on Manifolds. Graduate Studies in Mathematics 38. Amer. Math. Soc., Providence, RI.

[20] Kantorovich, L. V. (1948). On a problem of Monge. Uspekhi Mat. Nauk 3 225-226.

[21] Kantorovich, L. V. (1942). On mass transportation. Dokl. Akad. Nauk SSSR 37 227-229.

[22] Karatzas, I. and Shreve, S. E. (1991). Brownian Motion and Stochastic Calculus, 2nd ed. Graduate Texts in Mathematics 113. Springer, New York. MR1121940

[23] Kohn, R. V. and SERFaty, S. (2010). A deterministic-control-based approach to fully nonlinear parabolic and elliptic equations. Comm. Pure Appl. Math. 63 1298-1350.

[24] Lamberton, D., Pham, H. and Schweizer, M. (1998). Local risk-minimization under transaction costs. Math. Oper. Res. 23 585-612. MR1653777

[25] Lieberman, G. M. (1996). Second Order Parabolic Differential Equations. World Scientific Co., Inc., River Edge, NJ. MR1465184

[26] LiU, H. and YonG, J. M. (2005). Option pricing with an illiquid underlying asset market. J. Econom. Dynam. Control 29 2125-2156. 
[27] Loeper, G. (2006). The reconstruction problem for the Euler-Poisson system in cosmology. Arch. Ration. Mech. Anal. 179 153-216.

[28] PASCUCCI, A. (2011). PDE and Martingale Methods in Option Pricing, 1st ed. Springer, Milan. MR2791231

[29] Platen, E. and Schweizer, M. (1998). On feedback effects from hedging derivatives. Math. Finance 8 67-84.

[30] Rockafellar, R. T. (1997). Convex Analysis. Princeton Univ. Press, Princeton, NJ. Reprint of the 1970 original. MR1451876

[31] SchÖnbucher, P. J. and Wilmott, P. (2000). The feedback effect of hedging in illiquid markets. SIAM J. Appl. Math. 61 232-272. MR1776395

[32] Soner, H. M. and TOUZI, N. (2000). Superreplication under gamma constraints. SIAM J. Control Optim. 39 73-96.

[33] Soner, H. M. and Touzi, N. (2002). Dynamic programming for stochastic target problems and geometric flows. J. Eur. Math. Soc. (JEMS) 4 201-236.

[34] Soner, H. M. and TouzI, N. (2007). Hedging under gamma constraints by optimal stopping and face-lifting. Math. Finance 17 59-80.

[35] Soner, H. M., Touzi, N. and Zhang, J. (2013). Dual formulation of second order target problems. Ann. Appl. Probab. 23 308-347. MR3059237

[36] TAN, X. and TouZI, N. (2013). Optimal transportation under controlled stochastic dynamics. Ann. Probab. 41 3201-3240. MR3127880

[37] Tóth, B., Eisler, Z., Lillo, F., Kockelkoren, J., Bouchaud, J.-P. and Farmer, J. D. (2012). How does the market react to your order flow? Quant. Finance 12 1015-1024. MR2949509

[38] Tychonoff, A. (1935). Théorèmes d'unicité pour l'équation de la chaleur. Rec. Math. Moscou 42 199-216.

[39] VÁzQuEZ, J. L. (2006). Smoothing and decay estimates for nonlinear diffusion equations. In Equations of Porous Medium Type. Oxford Lecture Series in Mathematics and Its Applications 33. Oxford Univ. Press, Oxford.

[40] Villani, C. (2003). Topics in Optimal Transportation. Graduate Studies in Mathematics 58. Amer. Math. Soc., Providence, RI. MR1964483

[41] Wang, X.-J. (2006). Schauder estimates for elliptic and parabolic equations. Chin. Ann. Math. Ser. B 27 637-642.

\author{
SCHOOL of Mathematical Sciences \\ AND \\ Monash Centre For Quantitative Finance \\ AND INVESTMENT STRATEGIES \\ MONASH UNIVERSITY \\ CLAYTON, VIC 3800 \\ Australia \\ E-MAIL: gregoire.loeper@monash.edu
}

